OU-HET 599

February 2008

\title{
Fiber Bundles and Matrix Models
}

\author{
Takaaki Ishi $*$, Goro Ishikd \\ Department of Physics, Graduate School of Science \\ Osaka University, Toyonaka, Osaka 560-0043, Japan
}

\begin{abstract}
We investigate relationship between a gauge theory on a principal bundle and that on its base space. In the case where the principal bundle is itself a group manifold, we also study relations of those gauge theories with a matrix model obtained by dimensionally reducing them to zero dimensions. First, we develop the dimensional reduction of YangMills (YM) on the total space to YM-higgs on the base space for a general principal bundle. Second, we show a relationship that YM on an $S U(2)$ bundle is equivalent to the theory around a certain background of YM-higgs on its base space. This is an extension of our previous work [29], in which the same relationship concerning a $U(1)$ bundle is shown. We apply these results to the case of $S U(n+1)$ as the total space. By dimensionally reducing YM on $S U(n+1)$, we obtain YM-higgs on $S U(n+1) / S U(n) \simeq S^{2 n+1}$ and on $S U(n+1) /(S U(n) \times U(1)) \simeq C P^{n}$ and a matrix model. We show that the theory around each monopole vacuum of YM-higgs on $C P^{n}$ is equivalent to the theory around a certain vacuum of the matrix model in the commutative limit. By combining this with the relationship concerning a $U(1)$ bundle, we realize YM-higgs on $S U(n+1) / S U(n) \simeq S^{2 n+1}$ in the matrix model. We see that the relationship concerning a $U(1)$ bundle can be interpreted as Buscher's T-duality.
\end{abstract}

\footnotetext{
* e-mail address : ishii@het.phys.sci.osaka-u.ac.jp

$\dagger$ e-mail address : ishiki@het.phys.sci.osaka-u.ac.jp

$\ddagger$ e-mail address : shinji@het.phys.sci.osaka-u.ac.jp

$\S$ e-mail address : tsuchiya@het.phys.sci.osaka-u.ac.jp, address after April 2008 : Department of Physics, Shizuoka University, 836 Ohya, Suruga-ku, Shizuoka 422-8529, Japan
} 


\section{Contents}

1 Introduction and conclusion $\quad 1$

2 Typical relationships 6

3 Dimensional reduction on a principal bundle $\quad 13$

4 Extension of the matrix T-duality $\quad \mathbf{1 7}$

4.1 Nontrivial vacua and transformation between patches . . . . . . . . . . . . . . . . . .

$4.2 G=U(1), S U(2) \ldots \ldots \ldots \ldots \ldots \ldots$

4.3 Example: $S^{7} \rightarrow S^{4} \ldots \ldots \ldots \ldots$

5 Gauge theories on $S U(n+1)(/ H)$ and matrix model 25

5.1 Dimensional reduction of YM theory on a group manifold . . . . . . . . 26

5.2 Dimensional reduction of YM theory on $S U(n+1) \ldots \ldots$. . . . . . . . 28

5.3 Relations among gauge theories on $S U(n+1) / H \ldots \ldots$. . . . . . . 29

6 Interpretation as Buscher's T-duality

\begin{tabular}{|l|l|} 
A Spherical harmonics & 34 \\
\hline
\end{tabular}

B Derivation of (4.22)

C Group manifold and coset space $\quad 42$

D Metrics of $S U(3), S^{5}$ and $C P^{2} \quad 44$

\begin{tabular}{ll|l|} 
E Fuzzy $C P^{n}$ & 45 \\
\hline
\end{tabular}

\section{Introduction and conclusion}

Emergence of space-time is one of the key concepts in matrix models as nonperturbative definition of superstring [1-3]. This phenomenon was first observed in the relationship between a gauge theory and a matrix model. This is the so-called large $\mathrm{N}$ reduction [4]. It states that a large $N$ planar gauge theory is equivalent to the matrix model that is its dimensional reduction to zero dimensions unless the $U(1)^{D}$ symmetry is broken, where $D$ denotes the dimensionality of the original gauge theory. However, the $U(1)^{D}$ symmetry is in general spontaneously broken for $D>2$. There are two improved versions of the large $N$ reduced model that preserve the $U(1)^{D}$ symmetry. One is the quenched reduced model [5-8]. 
The other is the twisted reduced model [9], which was later rediscovered in the context of the noncommutative field theories [10]. The T-duality for D-brane effective theories [11], which we call the matrix T-duality in this paper, share the same idea with the large $\mathrm{N}$ reduced model. The statement of the matrix T-duality is that $U(N)$ Yang-Mills (YM) on $R^{p} \times S^{1}$ is equivalent to $U(N \times \infty)$ YM-higgs on $R^{p}$ which is a dimensional reduction of $U(N \times \infty)$ YM on $R^{p} \times S^{1}$ if a periodicity (orbifolding) condition is imposed. Also, deconstruction [12] and supersymmetric lattice gauge theories inspired by it [13] are analogs of the matrix Tduality. The above developments are all concerning gauge theories on flat space-time. It is important to understand how gauge theories on curved space-time are realized in matrix models or gauge theories in lower dimensions, because it would lead us to gain some insights into how curved space-time is realized in matrix models as nonperturbative definition of superstring. Note that an interesting approach to the description of curved spacetime by matrices was proposed in [14].

In [15], Takayama and three of the present authors found relationships among the $S U(2 \mid 4)$ symmetric theories. Here the $S U(2 \mid 4)$ symmetric theories include $\mathcal{N}=4$ super Yang Mills (SYM) on $R \times S^{3} / Z_{k}, 2+1 \mathrm{SYM}$ on $R \times S^{2}$ [16] and the plane wave matrix model (PWMM) [17]. These theories are related by dimensional reductions and possess common features: mass gap, discrete spectrum and many discrete vacua. From the gravity duals of those vacua proposed in [18], the following relations between these theories are suggested: the theory around each vacuum of $2+1 \mathrm{SYM}$ on $R \times S^{2}$ is equivalent to the theory around a certain vacuum of PWMM, and the theory around each vacuum of $\mathcal{N}=4 \mathrm{SYM}$ on $R \times S^{3} / Z_{k}$ is equivalent to the theory around a certain vacuum of $2+1 \mathrm{SYM}$ on $R \times S^{2}$ with the periodicity imposed. Combining these two equivalences, we can say that the theory around each vacuum of $\mathcal{N}=4 \mathrm{SYM}$ on $R \times S^{3} / Z_{k}$ is realized in PWMM. In [15], these equivalences were shown directly on the gauge theory side. The results in [15] not only serve as a nontrivial check of the gauge/gravity correspondence for the $S U(2 \mid 4)$ theories, but they are also interesting in the following aspects. Much work has been already done on the realization of the gauge theories on the fuzzy sphere [19-22] by matrix models [23] and on the monopoles on the fuzzy sphere [24-28]. Note that the realization of the fuzzy sphere by matrix models can be viewed as an extension of the twisted reduced model to curved space. Here in the relation between 2+1 SYM on $R \times S^{2}$ and PWMM, it was manifestly shown that the continuum limit of concentric fuzzy spheres correspond to multi monopoles. The relation between $\mathcal{N}=4$ SYM on $R \times S^{3} / Z_{k}$ and $2+1$ SYM on $R \times S^{2}$ can be regarded as an extension of the matrix T-duality to that on a nontrivial $U(1)$ bundle, $S^{3} / Z_{k}$, whose base space is $S^{2}$. Furthermore, in [29], we generalized the matrix T-duality to that on an arbitrary $U(1)$ bundle. As an application of these results, in [30], Ohta and the present authors investigated relationships 
among Chern-Simons theory on a $U(1)$ bundle over a Riemann surface, BF theory with a mass term on the Riemann surface, which is equivalent to two-dimensional Yang-Mills on the Riemann surface, and a matrix model. It was discussed that the former two (topological) field theories associated with topological strings can be realized in the matrix model. The results in [15] also suggests an interesting possibility of a nonperturbative formulation of $\mathcal{N}=4 \mathrm{SYM}$ on $R \times S^{3}$ by PWMM, which would lead to a nonperturbative test of the AdS/CFT correspondence.

This paper is aimed at further investigation of the above developments concerning the large $N$ reduction and the matrix T-duality on curved space. First, we develop a dimensional reduction of YM on the total space to YM-higgs on the base space for a general principal bundle. This also enables us to dimensionally reduce YM on a group manifold to a matrix model. Second, as an extension of the work [29], in the case in which the fiber is $S U(2)$, we show that YM on the total space is equivalent to a certain vacuum 1 of YM-higgs on the base space with the periodicity imposed. This enables us to realize YM on an $S U(2)^{k} \times U(1)^{l}$ bundle in YM-higgs on its base space. We apply the above results to the case of $S U(n+1)$ as the total space. $S U(n+1)$ is viewed as $S U(n)$ bundle over $S U(n+1) / S U(n) \simeq S^{2 n+1}$ or $S U(n) \times U(1)$ bundle over $S U(n+1) /(S U(n) \times U(1)) \simeq C P^{n}$, and $S U(n+1) / S U(n) \simeq S^{2 n+1}$ is viewed as $U(1)$ bundle over $C P^{n}$. By the dimensional reduction, we obtain YM-higgs on $S^{2 n+1}$ and $C P^{n}$ and a matrix model. We find the commutative (continuum) limit of gauge theory on fuzzy $C P^{n}[28,31-35]$ realized in the matrix model coincides with YM-higgs on $C P^{n}$. Namely, we show that the theory around each monopole vacuum of YM-higgs on $C P^{n}$ is equivalent to the theory around a certain vacuum of the matrix model. By combing this with the extended matrix T-duality, we realize YM-higgs on $S U(n+1) / S U(n) \simeq S^{2 n+1}$ in the matrix model. We also show that the extended matrix T-duality of the $U(1)$ case developed in [29] can be interpreted as Buscher's T-duality [36].

In the remainder of this section, we describe the organization of the present paper, providing our results in detail, and finally describe some outlook. From the same reasoning as the case of the $S U(2 \mid 4)$ symmetric theories, the following relationships among YM on $S^{3}$, YM-higgs on $S^{2}$ and a matrix model hold. These theories are related to each other by dimensional reductions. The theory around each vacuum of YM-higgs on $S^{2}$ is equivalent to the theory around a certain vacuum of the matrix model. YM on $S^{3}$ is equivalent to the theory around a certain vacuum of YM-higgs on $S^{2}$ with the periodicity imposed. Eventually, YM on $S^{3}$ is realized in the matrix model. It can be said that our results in this paper are extension of these relationships. In section 2, we show these relationships in order to illustrate our basic ideas.

\footnotetext{
${ }^{1}$ Throughout this paper, we consider gauge theories on manifolds with the Euclidean signature. Here 'vacuum' represents a configuration that gives the global minimum of the classical action.
} 


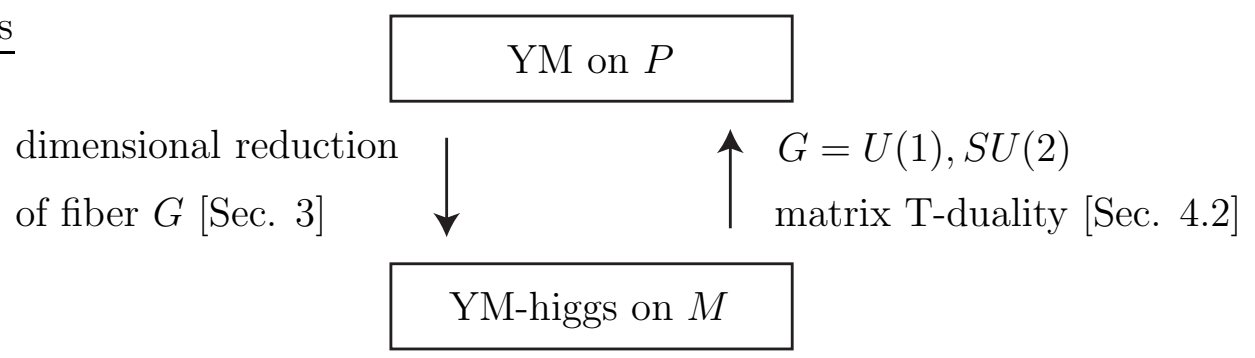

Figure 1: Matrix T-duality for $G=U(1), S U(2)$

In section 3, we develop a dimensional reduction on a general principal fiber bundle. We start with YM on the total space, dimensionally reduce the fiber directions and obtain a YM-higgs on the base space.

In section 4, we examine a relationship between YM on the total space and YM-higgs on the base space obtained in section 3. In section 4.1, we first examine the transformations of the fields from a local patch to another local patch in YM-higgs on the base space. In section 4.2 , using the observation in section 4.1, we show that when the fiber is $U(1)$ or $S U(2)$, YM on the total space is equivalent to the theory around a certain vacuum of YM-higgs on the base space with the periodicity imposed. This vacuum is given by multimonopole configuration on the base space. We already found the $U(1)$ case of this equivalence in [29]. In the $S U(2)$ case, we also use the result in section 2 that $\mathrm{YM}$ on $S^{3}$ is realized in the matrix model. As a generalization, we realize YM on $S U(2)^{k} \times U(1)^{l}$ bundle in YM-higgs on its base space. In section 4.3, as an example, we consider $S^{7}$ which is an $S U(2)$ bundle over $S^{4}$. In Fig. 1, we summarize our results in sections 3 and 4 .

In section 5 , we examine a series of $S U(n+1)$ symmetric theories. Fig. 2 summarizes our findings in section 5 and their relation to other sections. The case of $n=1$ is nothing but the example discussed in section 2. In this case, YM on $S U(2)$ is the same as YM-higgs on $S^{3}$ because $S U(2) \simeq S^{3}$. In section 5.1, as a special case of section 3, we consider a dimensional reduction of YM on a group manifold $\tilde{G}$ to a coset space $\tilde{G} / H$ where $H$ is a subgroup of $\tilde{G}$. Namely, we view $\tilde{G}$ as an $H$ bundle over $\tilde{G} / H$. By dimensionally reducing the Killing vectors on $\tilde{G}$ to those on $\tilde{G} / H$, we obtain a theory on $\tilde{G} / H$ expressed in terms of the Killing vectors. Then, we show that this theory on $\tilde{G} / H$ is rewritten into YM-higgs on $\tilde{G} / H$ obtained in section 3. In section 5.2, we apply the results in section 5.1 to the case of $\tilde{G}=S U(n+1)$ and obtain a series of theories in Fig. 2 which possess $S U(n+1)$ symmetry. If we take $S U(n)$ as $H$, we obtain YM-higgs on $S^{2 n+1}$. Note that the isometry of this $S^{2 n+1}$ is not $S O(2 n+2)$ but $S U(n+1)$. For $n \geq 2$, it is different from the ordinary $S^{2 n+1}$ but homeomorphic to the ordinary one, and is called a squashed $S^{2 n+1}$. If we take 


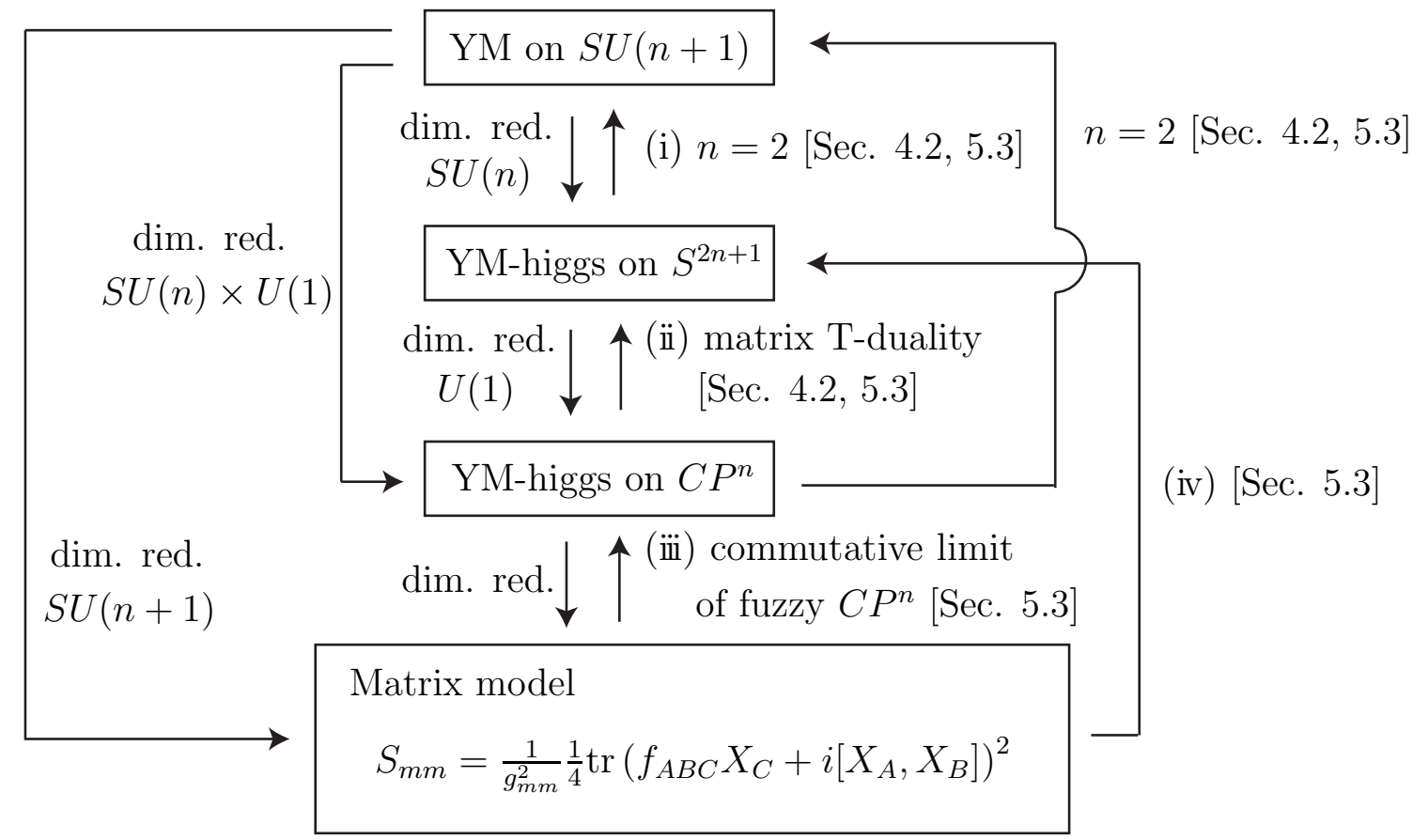

Figure 2: A series of theories studied in section 5.

$S U(n) \times U(1)$ as $H$, we obtain YM-higgs on $C P^{n}$. Finally if we take $S U(n+1)$ itself as $H$, we obtain a matrix model whose action is shown in Fig. 2, where $f_{A B C}$ is the structure constant of the $S U(n+1)$ Lie algebra. As indicated in Fig. 2, these dimensional reductions can also be performed step by step: we obtain YM-higgs on $C P^{n}$ from YM-higgs on $S^{2 n+1}$ and the matrix model from YM-higgs on $C P^{n}$. In the case of $n=2$, as an application of the result in section 4.2, we see that YM on $S U(3)$ is equivalent to the theory around a vacuum of YM-higgs on $S^{5}$ with the periodicity imposed ((i) in Fig. 2). Since $S^{2 n+1}$ can be viewed as a $U(1)$ bundle over $C P^{n}$, in section 5.3, we show as an application of the results in section 4.2 that the theory around each vacuum of YM-higgs on $S^{2 n+1}$ is equivalent to the theory around a vacuum of YM-higgs on $C P^{n}$ with the periodicity imposed ((ii) in Fig. 2). In section 5.3, we show that the theory around each abelian monopole vacuum of YM-higgs on $C P^{n}$ is equivalent to a certain vacuum of the matrix model ((iii) in Fig. 2). Combining these results, we also show that the theory around the trivial vacuum of YM-higgs on $S^{2 n+1}$ is realized in the matrix model ((iv) in Fig. 2). YM on $S U(3)$ is realized in YM-higgs on $C P^{2}((\mathrm{v})$ in Fig. 2). Finally, we make a comment: it follows from the result in section 4 that YM on $S U(n+1)$ is realized in YM-higgs on $S U(n+1) /\left(S U(2)^{k} \times U(1)^{l}\right)$.

In section 6, we discuss how the extended matrix T-dulaity found in [29] and reviewed in section 4.2 is interpreted as Buscher's T-duality. In appendices A-D, we describe some 
details.

It is an open problem whether YM on $S U(n+1)$ with $n \geq 2$ is realized in the matrix model. Presumably, we need to construct noncommutative counterparts of non-Abelian monopoles of YM-higgs on $S^{2 n+1}$ or $C P^{n}$ in the matrix model. Realization of YM on $S U(n+1)$ in the matrix model should enable us to extend the matrix T-duality to the case of $G=S U(n+1)$. Of course, the matrix T-duality for a general $G$ should still be investigated. It is important to see whether the matrix T-duality in the $S U(2)$ case is associated with the nonabelian T-duality discussed within the nonlinear sigma models [37]. It is also relevant to identify the commutative limit of the matrix model consisting of the square of the commutators and the generalized Myers term with the $S U(n+1)$ structure constant which has been examined in $[33,38]$ and find its higher-dimensional origin. Analysis in this paper is classical. Whether the relationships among the gauge theories we found hold quantum mechanically is a nontrivial and important problem. It should be noted that in the quantum correspondence no orbifolding condition is needed in the matrix T-duality as far as the planar limit is concerned. This is nothing but the large $N$ reduction and enables us to make the size of matrices become finite and play a role of the ultraviolet cutoff. In particular, we expect to give a nonperturbative definition of $\mathcal{N}=4 \mathrm{SYM}$ on $R \times S^{3}$ in the planar limit in terms of PWMM [39].

\section{Typical relationships}

In this section, to illustrate our ideas, we describe relationships among YM on $S^{3}$, YM-higgs on $S^{2}$ and a matrix model. These relationships are essentially the same as those among the $S U(2 \mid 4)$ symmetric theories found in [15].

We consider $S^{3}$ with radius $2 / \mu$ and regard it as the $U(1)\left(S^{1}\right)$ Hopf bundle on $S^{2}$ with radius $1 / \mu$. $S^{3}$ with radius $2 / \mu$ is defined by

$$
\left\{\left.\left(w_{1}, w_{2}\right) \in C^{2}|| w_{1}\right|^{2}+\left|w_{2}\right|^{2}=4 / \mu^{2}\right\}
$$

The Hopf map $\pi: S^{3} \rightarrow C P^{1}\left(S^{2}\right)$ is defined by

$$
\left(w_{1}, w_{2}\right) \rightarrow\left[\left(w_{1}, w_{2}\right)\right] \equiv\left\{\lambda\left(w_{1}, w_{2}\right) \mid \lambda \in C \backslash\{0\}\right\}
$$

Two patches are introduced on $C P^{1}$ : the patch I $\left(w_{1} \neq 0\right)$ and the patch II $\left(w_{2} \neq 0\right)$. On the patch I the local trivialization is given by

$$
\left(w_{1}, w_{2}\right) \rightarrow\left(\frac{w_{2}}{w_{1}}, \frac{w_{1}}{\left|w_{1}\right|}\right) \in(\text { patch } \mathrm{I}) \times U(1)
$$


while on the patch II the local trivialization is given by

$$
\left(w_{1}, w_{2}\right) \rightarrow\left(\frac{w_{1}}{w_{2}}, \frac{w_{2}}{\left|w_{2}\right|}\right) \in(\text { patch II }) \times U(1)
$$

The equation (2.1) is solved as

$$
w_{1}=\frac{2}{\mu} \cos \frac{\theta}{2} e^{i \sigma_{1}}, \quad w_{2}=\frac{2}{\mu} \sin \frac{\theta}{2} e^{i \sigma_{2}}
$$

where $0 \leq \theta \leq \pi$ and $0 \leq \sigma_{1}, \sigma_{2}<2 \pi$. We put

$$
\varphi=\sigma_{1}-\sigma_{2}, \quad \psi=\sigma_{1}+\sigma_{2}
$$

and can change the ranges of $\varphi$ and $\psi$ to $0 \leq \varphi<2 \pi$ and $0 \leq \psi<4 \pi$. The periodicity is expressed as

$$
(\theta, \varphi, \psi) \sim(\theta, \varphi+2 \pi, \psi+2 \pi) \sim(\theta, \varphi, \psi+4 \pi)
$$

From the local trivializations (2.3) and (2.4), one can see that $\theta$ and $\varphi$ are regarded as the angular coordinates of the base space $S^{2}$ through the stereographic projection. The patch I corresponds to $0 \leq \theta<\pi$, while the patch II corresponds to $0<\theta \leq \pi$. The metric of $S^{3}$ is given as follows:

$$
\begin{aligned}
d s_{S^{3}}^{2} & =\left|d w_{1}\right|^{2}+\left|d w_{2}\right|^{2} \\
& =\frac{1}{\mu^{2}}\left(d \theta^{2}+\sin ^{2} \theta d \varphi^{2}+(d \psi+\cos \theta d \varphi)^{2}\right) .
\end{aligned}
$$

In the remainder of this section, the upper sign is taken in the patch I and the lower sign in the patch II. From (2.3), (2.4) and (2.8), one sees that the fiber $S^{1}$ is parameterized by $y=\frac{1}{\mu}(\psi \pm \varphi)$ and its radius is given by $2 / \mu$. The connection 1 -form is given by

$$
\omega=\frac{\mu}{2}\left(d y+\frac{1}{\mu}(\cos \theta \mp 1) d \varphi\right)
$$

The connection 1-form provides the vertical-horizontal decomposition by determining the inverse of the dreibein $E_{A}^{M}$ through $\omega\left(E_{\alpha}^{M}\right)=0, E_{3}^{\mu}=0$ and $E_{3}^{y}=1$, where $A=1,2,3$, $\alpha=1,2, M=\theta, \varphi, y$ and $\mu=\theta, \varphi$. The inverse of the dreibein is determined as

$$
\begin{aligned}
& E_{1}^{\theta}=\mu, \quad E_{2}^{\varphi}=\frac{\mu}{\sin \theta}, \\
& E_{2}^{y}=\mu \frac{\cos \theta \mp 1}{\sin \theta}, \quad E_{3}^{y}=1, \\
& \text { others }=0 .
\end{aligned}
$$


The dreibein are given by

$$
\begin{aligned}
& E_{\theta}^{1}=e_{\theta}^{1}=\frac{1}{\mu}, \quad E_{\varphi}^{2}=e_{\varphi}^{2}=\frac{1}{\mu} \sin \theta, \\
& E_{\varphi}^{3}=\frac{1}{\mu}(\cos \theta \mp 1), \quad E_{y}^{3}=1, \\
& \text { others }=0,
\end{aligned}
$$

where $e_{\mu}^{\alpha}$ are the zweibein of $S^{2}$.

We start with YM on $S^{3}$

$$
S_{S^{3}}=\frac{1}{4 g_{S^{3}}^{2}} \int \frac{d \Omega_{3}}{(\mu / 2)^{3}} \operatorname{tr}\left(F_{A B} F_{A B}\right) .
$$

The vertical-horizontal decomposition tells us how to relate the gauge field on $S^{3}$ to the gauge field and the higgs field on $S^{2}$ :

$$
\begin{aligned}
& A_{\alpha}=a_{\alpha}, \\
& A_{3}=\phi .
\end{aligned}
$$

Or equivalently

$$
\begin{aligned}
& A_{\theta}=a_{\theta}, \\
& A_{\varphi}=a_{\varphi}+\frac{1}{\mu}(\cos \theta \mp 1) \phi, \\
& A_{y}=\phi .
\end{aligned}
$$

In (2.13) and (2.14), in order to make a dimensional reduction, we assume that the both sides are independent of $y$. Then, substituting (2.13) into (2.12) yields a YM-higgs on $S^{2}$,

$$
S_{S^{2}}=\frac{1}{g_{S^{2}}^{2}} \int \frac{d \Omega_{2}}{\mu^{2}} \operatorname{tr}\left(\frac{1}{2}\left(f_{12}+\mu \phi\right)^{2}+\frac{1}{2}\left(D_{\alpha} \phi\right)^{2}\right),
$$

where $g_{S^{2}}^{2}=\frac{\mu}{4 \pi} g_{S^{3}}^{2}$. It is convenient for us to rewrite (2.15) using the three-dimensional flat space notation. We define a three-dimensional vector field in terms of $a_{\alpha}$ and $\phi$ [16]:

$$
\vec{X}=\phi \vec{e}_{r}+a_{1} \vec{e}_{\varphi}-a_{2} \vec{e}_{\theta}
$$

where $\vec{e}_{r}=(\sin \theta \cos \varphi, \sin \theta \sin \varphi, \cos \theta)$ and $\vec{e}_{\theta}=\frac{\partial \vec{e}_{r}}{\partial \theta}, \vec{e}_{\varphi}=\frac{1}{\sin \theta} \frac{\partial \vec{e}_{r}}{\partial \varphi}$. We also introduce the angular momentum operator in three-dimensional flat space,

$$
\vec{L}^{(0)}=-i \vec{e}_{\phi} \partial_{\theta}+i \frac{1}{\sin \theta} \vec{e}_{\theta} \partial_{\phi}
$$


Then, (2.15) is rewritten as

$$
S_{S^{2}}=\frac{1}{g_{S^{2}}^{2}} \int \frac{d \Omega_{2}}{\mu^{2}} \frac{1}{2} \operatorname{tr}\left(\mu X_{A}+i \mu \epsilon_{A B C} L_{B}^{(0)} X_{C}+\frac{i}{2} \epsilon_{A B C}\left[X_{B}, X_{C}\right]\right)^{2} .
$$

By dropping all the derivatives, we dimensionally reduce (2.18) to zero dimensions to obtain a matrix model:

$$
S_{m m}=\frac{1}{g_{m m}^{2}} \frac{1}{2} \operatorname{tr}\left(\mu X_{A}+\frac{i}{2} \epsilon_{A B C}\left[X_{B}, X_{C}\right]\right)^{2}
$$

where $g_{m m}^{2}=\frac{\mu^{2}}{4 \pi} g_{S^{2}}^{2}$. The cross term in the above action is nothing but the Myers term [40]. It was first found in [41] that (2.19) is obtained from (2.12) through the dimensional reduction.

We can obtain (2.18) and (2.19) directly from (2.12) in the following way. We parameterize the gauge field on $S^{3}$ as $A=X_{A} E^{A}$ [18], where $E^{A}$ is the right invariant 1-form defined in appendix A. Then, by using the Maurer-Cartan equation (A.4), we evaluate the curvature 2 -form as

$$
\begin{aligned}
F & =d A+i A \wedge A \\
& =\frac{1}{2} \epsilon_{A B C}\left(i \mu \epsilon_{C D E} \mathcal{L}_{D} X_{E}+\mu X_{C}+i \epsilon_{C D E} X_{D} X_{E}\right) E^{A} \wedge E^{B},
\end{aligned}
$$

where $\mathcal{L}_{A}$ are the Killing vector dual to $E^{A}$, the explicit form of which is given in (A.7). Noting that $\mathcal{L}_{A}$ reduces to $L_{A}^{(0)}$ when $X_{A}$ is independent of $y$, one can easily see that (2.12) is dimensionally reduced to (2.18). Moreover, if we assume that $X_{A}$ is independent of all coordinates, we obtain the matrix model (2.19) directly from (2.12).

The theories (2.15) and (2.19) possess many nontrivial vacua. Let us see how those vacua are described. First, the vacuum configurations of (2.15) with the gauge group $U(M)$ are determined by

$$
\begin{aligned}
& f_{12}+\mu \phi=0 \\
& D_{\alpha} \phi=0 .
\end{aligned}
$$

In the gauge in which $\phi$ is diagonal, (2.21) is solved as

$$
\begin{aligned}
& \hat{a}_{1}=0 \\
& \hat{a}_{2}=\frac{\cos \theta \mp 1}{\sin \theta} \hat{\phi}, \\
& \hat{\phi}=\frac{\mu}{2} \operatorname{diag}(\cdots, \underbrace{n_{s-1}, \cdots, n_{s-1}}_{N_{s-1}}, \underbrace{n_{s}, \cdots, n_{s}}_{N_{s}}, \underbrace{n_{s+1}, \cdots, n_{s+1}}_{N_{s+1}}, \cdots),
\end{aligned}
$$


where the gauge field takes the configurations of Dirac's monopoles, so that $n_{s}$ must be integers due to Dirac's quantization condition. Note also that $\sum_{s} N_{s}=M$. Thus the vacua of YM-higgs on $S^{2}$ are classified by the monopole charges $n_{s} / 2$ and their degeneracies $N_{s}$. Next, the vacuum configurations of (2.19) with the gauge group $U(\hat{M})$ are determined by 2

$$
\left[X_{A}, X_{B}\right]=i \mu \epsilon_{A B C} X_{C}
$$

(2.23) is solved as

$$
\hat{X}_{A}=\mu L_{A}
$$

where $L_{A}$ are the representation matrices of the $S U(2)$ generators which are in general reducible, and are decomposed into irreducible representations:

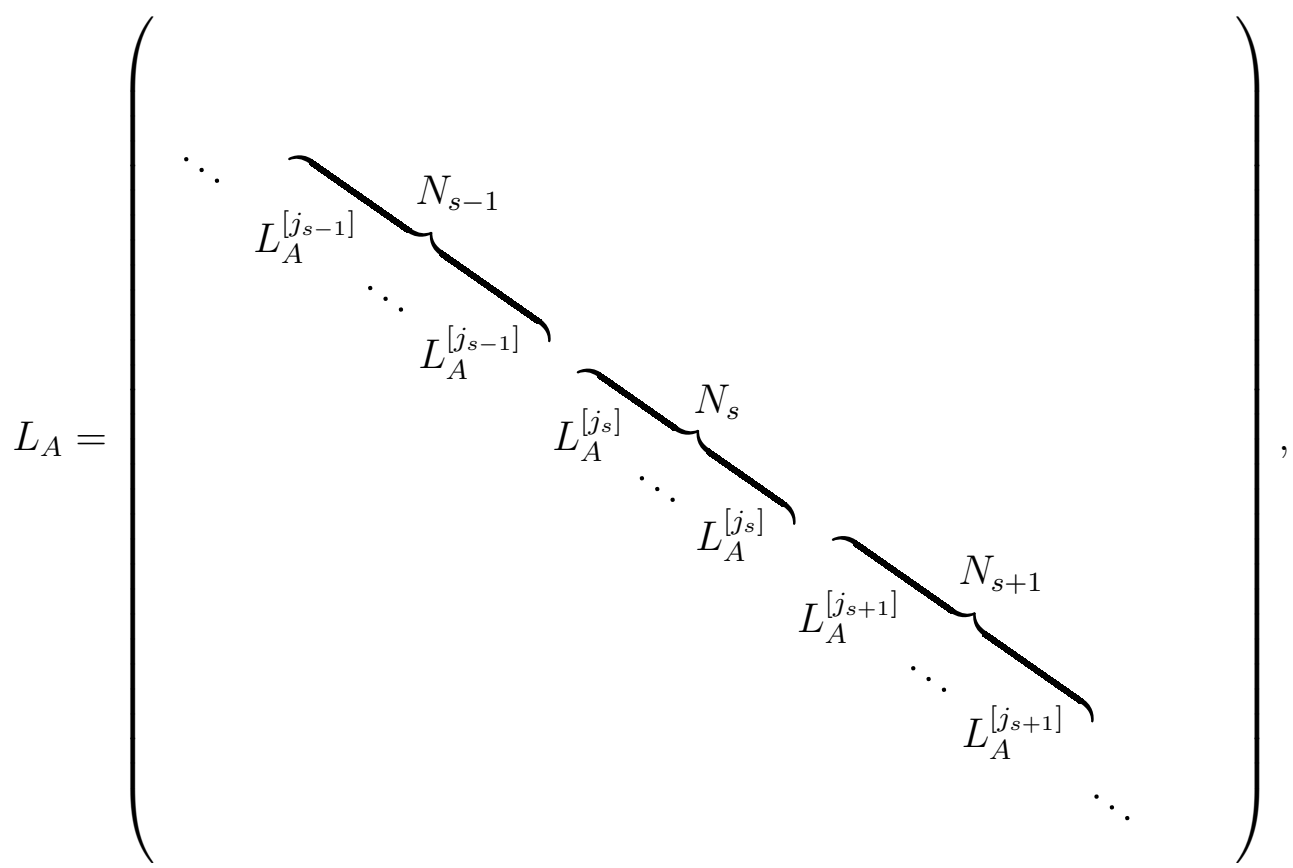

where $L_{A}^{[j]}$ are the spin $j$ representation matrices of $S U(2)$ and $\sum_{s} N_{s}\left(2 j_{s}+1\right)=\hat{M}$. The vacua of the matrix model are classified by the $S U(2)$ representations $\left[j_{s}\right]$ and their degeneracies $N_{s}$. (2.25) represents concentric fuzzy spheres with different radii.

In the remainder of this section, we show relationships among the theories (2.12), (2.15) and (2.19). First, we show that the theory around the vacuum (2.22) of YM-higgs on $S^{2}$ is equivalent to the theory around the vacuum (2.24) of the matrix model if one puts $2 j_{s}+1=$ $N_{0}+n_{s}$ and takes the $N_{0} \rightarrow \infty$ limit with $g_{m m}^{2} / N_{0}$ fixed to $g_{S^{2}}^{2} \mu^{2} / 4 \pi$. We decompose the

\footnotetext{
${ }^{2}$ There is a solution to the equations of motion of the matrix model (2.19), $X_{A}=\frac{\mu}{2} L_{A}$, which does not satisfy (2.23). It turns out that the theory around this solution is unstable.
} 
fields into the background corresponding (2.22) and the fluctuation as $X_{A}^{(s, t)} \rightarrow \hat{X}_{A}^{(s, t)}+X_{A}^{(s, t)}$, where $(s, t)$ label the (off-diagonal) blocks. Then, (2.18) is expanded around (2.22) as

$$
\begin{aligned}
S_{S^{2}}=\frac{1}{g_{S^{2}}^{2}} \int \frac{d \Omega_{2}}{\mu^{2}} \frac{1}{2} \sum_{s, t} \operatorname{tr} & {\left[\left(\mu X_{A}^{(s, t)}+i \mu \epsilon_{A B C} L_{B}^{\left(q_{s t}\right)} X_{C}^{(s, t)}+\frac{i}{2} \epsilon_{A B C}\left[X_{B}, X_{C}\right]^{(s, t)}\right)\right.} \\
& \left.\times\left(\mu X_{A}^{(t, s)}+i \mu \epsilon_{A D E} L_{D}^{\left(q_{t s}\right)} X_{E}^{(t, s)}+\frac{i}{2} \epsilon_{A D E}\left[X_{D}, X_{E}\right]^{(t, s)}\right)\right],
\end{aligned}
$$

where $q_{s t}=\left(n_{s}-n_{t}\right) / 2 . \vec{L}^{(q)}$ is the angular momentum operator in the presence of a monopole with the magnetic charge $q$ at the origin, which takes the form [42]

$$
\vec{L}^{(q)}=\vec{L}^{(0)}-q \frac{\cos \theta \mp 1}{\sin \theta} \vec{e}_{\theta}-q \vec{e}_{r} .
$$

We make a harmonic expansion of $(2.26)$ by expanding the fluctuation in terms of the monopole vector spherical harmonics $\tilde{Y}_{J m q A}^{\rho}$ defined in appendix A as

$$
X_{A}^{(s, t)}=\sum_{\rho=0, \pm 1} \sum_{\tilde{Q} \geq\left|q_{s t}\right|} \sum_{m=-Q}^{Q} X_{J m \rho}^{(s, t)} \tilde{Y}_{J m q A}^{\rho}
$$

where $Q=J+\frac{(1+\rho) \rho}{2}$ and $\tilde{Q}=J-\frac{(1-\rho) \rho}{2}$. Substituting (2.28) into (2.26) yields

$$
\begin{aligned}
& S_{S^{2}}=\frac{4 \pi}{g_{S^{2}}^{2} \mu^{2}} \operatorname{tr}\left[\frac{\mu^{2}}{2} \sum_{s, t} \rho^{2}(J+1)^{2} X_{J m \rho}^{(s, t) \dagger} X_{J m \rho}^{(s, t)}\right. \\
& +i \mu \sum_{s, t, u} \rho_{1}\left(J_{1}+1\right) \mathcal{E}_{J_{1} m_{1} q_{s t} \rho_{1} J_{2} m_{2} q_{t u} \rho_{2} J_{3} m_{3} q_{u s} \rho_{3}} X_{J_{1} m_{1} \rho_{1}}^{(s, t)} X_{J_{2} m_{2} \rho_{2}}^{(t, u)} X_{J_{3} m_{3} \rho_{3}}^{(u, s)} \\
& -\frac{1}{2} \sum_{s, t, u, v}(-1)^{m-q_{s u}+1} \mathcal{E}_{J-m q_{u s} \rho J_{1} m_{1} q_{s t} \rho_{1} J_{2} m_{2} q_{t u} \rho_{2}} \mathcal{E}_{J m q_{s u} \rho J_{3} m_{3} q_{u v} \rho_{3} J_{4} m_{4} q_{v s} \rho_{4}} \\
& \left.\quad \times X_{J_{1} m_{1} \rho_{1}}^{(s, t)} X_{J_{2} m_{2} \rho_{2}}^{(t, u)} X_{J_{3} m_{3} \rho_{3}}^{(u, v)} X_{J_{4} m_{4} \rho_{4}}^{(v, s)}\right],
\end{aligned}
$$

where $\mathcal{E}_{J_{1} m_{1} q_{s t} \rho_{1} J_{2} m_{2} q_{t u} \rho_{2} J_{3} m_{3} q_{u s} \rho_{3}}$ is defined in (A.40) and we have used (A.37). Similarly we decompose the matrices into the background given by (2.24) and the fluctuation as $X_{i} \rightarrow \hat{X}_{i}+X_{i}$ and obtain the theory around (2.24):

$$
\begin{aligned}
S_{m m}=\frac{1}{g_{m m}^{2}} \frac{1}{2} \sum_{s, t} \operatorname{tr}[ & \left(\mu X_{A}^{(s, t)}+i \mu \epsilon_{A B C} L_{B} \circ X_{C}^{(s, t)}+\frac{i}{2} \epsilon_{A B C}\left[X_{B}, X_{C}\right]^{(s, t)}\right) \\
\times & \left.\left(\mu X_{A}^{(t, s)}+i \mu \epsilon_{A D E} L_{D} \circ X_{E}^{(t, s)}+\frac{i}{2} \epsilon_{A D E}\left[X_{D}, X_{E}\right]^{(t, s)}\right)\right],
\end{aligned}
$$


where $L_{A}$ ○ is defined by

$$
L_{A} \circ X_{B}^{(s, t)}=L_{A}^{\left[j_{s}\right]} X_{B}^{(s, t)}-X_{B}^{(s, t)} L_{A}^{\left[j_{t}\right]} .
$$

We make a harmonic expansion for (2.30) by expanding the fluctuation in terms of the fuzzy vector spherical harmonics $\hat{Y}_{J m\left(j_{s} j_{t}\right) A}^{\rho}$ defined in appendix A as

$$
X_{A}^{(s, t)}=\sum_{\rho=0, \pm 1} \sum_{\tilde{Q} \geq\left|j_{s}-j_{t}\right|}^{j_{s}+j_{t}} \sum_{m=-Q}^{Q} X_{J m \rho}^{(s, t)} \otimes \hat{Y}_{J m\left(j_{s} j_{t}\right) A}^{\rho} .
$$

Since $j_{s}+j_{t}=N_{0}+\frac{n_{s}+n_{t}}{2}-1, N_{0}$ plays a role of the ultraviolet cutoff. Note also that $j_{s}-j_{t}=\left(n_{s}-n_{t}\right) / 2=q_{s t}$. Substituting (2.32) into (2.30) yields

$$
\begin{aligned}
& S_{m m}=\frac{N_{0}}{g_{m m}^{2}} \operatorname{tr}\left[\frac{\mu^{2}}{2} \sum_{s, t} \rho^{2}(J+1)^{2} X_{J m \rho}^{(s, t) \dagger} X_{J m \rho}^{(s, t)}\right. \\
& +i \mu \sum_{s, t, u} \rho_{1}\left(J_{1}+1\right) \hat{\mathcal{E}}_{J_{1} m_{1}\left(j_{s} j_{t}\right) \rho_{1} J_{2} m_{2}\left(j_{t} j_{u}\right) \rho_{2} J_{3} m_{3}\left(j_{u} j_{s}\right) \rho_{3}} X_{J_{1} m_{1} \rho_{1}}^{(s, t)} X_{J_{2} m_{2} \rho_{2}}^{(t, u)} X_{J_{3} m_{3} \rho_{3}}^{(u, s)} \\
& -\frac{1}{2} \sum_{s, t, u, v}(-1)^{m-q_{s u}+1} \hat{\mathcal{E}}_{J-m\left(j_{u} j_{s}\right) \rho J_{1} m_{1}\left(j_{s} j_{t}\right) \rho_{1} J_{2} m_{2}\left(j_{t} j_{u}\right) \rho_{2}} \hat{\mathcal{E}}_{J m\left(j_{s} j_{u}\right) \rho J_{3} m_{3}\left(j_{u} j_{v}\right) \rho_{3} J_{4} m_{4}\left(j_{v} j_{s}\right) \rho_{4}} \\
& \left.\times X_{J_{1} m_{1} \rho_{1}}^{(s, t)} X_{J_{2} m_{2} \rho_{2}}^{(t, u)} X_{J_{3} m_{3} \rho_{3}}^{(u, v)} X_{J_{4} m_{4} \rho_{4}}^{(v, s)}\right],
\end{aligned}
$$

where $\hat{\mathcal{E}}_{J_{1} m_{1}\left(j_{s} j_{t}\right) \rho_{1} J_{2} m_{2}\left(j_{t} j_{u}\right) \rho_{2} J_{3} m_{3}\left(j_{u} j_{s}\right) \rho_{3}}$ is defined in (A.40) and we have used (A.37). In the $N_{0} \rightarrow \infty$ limit, the ultraviolet cutoff goes to infinity and $\hat{\mathcal{E}}_{J_{1} m_{1}\left(j_{s} j_{t}\right) \rho_{1} J_{2} m_{2}\left(j_{t} j_{u}\right) \rho_{2} J_{3} m_{3}\left(j_{u} j_{s}\right) \rho_{3}}$ reduces to $\mathcal{E}_{J_{1} m_{1} q_{s t} \rho_{1} J_{2} m_{2} q_{t u} \rho_{2} J_{3} m_{3} q_{u s} \rho_{3}}$ as shown in appendix A. Namely, this limit corresponds to the commutative (continuum) limit of the fuzzy spheres. Hence, in the limit in which $N_{0} \rightarrow \infty$ and $g_{m m} \rightarrow \infty$ such that $g_{m m}^{2} / N_{0}=g_{S^{2}}^{2} \mu^{2} / 4 \pi$, (2.33) agrees with (2.29). We have proven our statement.

Next, we show that the theory around a certain vacuum of $U(M=N \times \infty)$ YM-higgs on $S^{2}$ with a periodicity condition imposed is equivalent to $U(N)$ YM on $S^{3}$. This is an extension of the matrix T-duality to a nontrivial fiber bundle. The vacuum of YM-higgs on $S^{2}$ we take is given by (2.22) with $s$ running from $-\infty$ to $\infty, n_{s}=s$ and $N_{s}=N .4 \pi g_{S^{2}}^{2} / \mu$ is identified with the coupling constant on $S^{3}, g_{S^{3}}^{2}$. We decompose the fields on $S^{2}$ into the background and the fluctuation,

$$
\begin{aligned}
& a_{\alpha} \rightarrow \hat{a}_{\alpha}+a_{\alpha}, \\
& \phi \rightarrow \hat{\phi}+\phi,
\end{aligned}
$$

and impose the periodicity (orbifolding) condition on the fluctuation,

$$
a_{\alpha}^{(s+1, t+1)}=a_{\alpha}^{(s, t)} \equiv a_{\alpha}^{(s-t)},
$$




$$
\phi^{(s+1, t+1)}=\phi^{(s, t)} \equiv \phi^{(s-t)} .
$$

The fluctuations are gauge-transformed from the patch I to the patch II as [29]

$$
\begin{aligned}
a_{\alpha}^{\prime(s-t)} & =e^{-i(s-t) \phi} a_{\alpha}^{(s-t)}, \\
\phi^{\prime(s-t)} & =e^{-i(s-t) \phi} \phi^{(s-t)} .
\end{aligned}
$$

We make the Fourier transformation for the fluctuations on each patch to construct the gauge field on the total space from the fields on the base space:

$$
\begin{aligned}
& A_{\alpha}(\theta, \varphi, \psi)=\sum_{w} a_{\alpha}^{(w)}(\theta, \varphi) e^{-i \frac{\mu}{2} w y}, \\
& A_{y}(\theta, \varphi, \psi)=\sum_{w} \phi^{(w)}(\theta, \varphi) e^{-i \frac{\mu}{2} w y} .
\end{aligned}
$$

We see from (2.36) that the lefthand sides of (2.37) are indeed independent of the patches. We substitute (2.37) into (2.26) and divide an overall factor $\sum_{s}$ to extract a single period. Then, we obtain $U(N)$ YM on $S^{3}$. The details of this calculation are given as a special case of (4.13) and (4.14).

Finally, combining the above two statements, we see that the theory around (2.24) of the matrix model where $s$ runs from $-\infty$ to $\infty, 2 j_{s}+1=N_{0}+s$ is equivalent to $U(N)$ YM on $S^{3}$ if the $N_{0} \rightarrow \infty$ limit is taken with $g_{m m}^{2} / N_{0}$ fixed to $\frac{g_{S 3}^{2} \mu^{3}}{16 \pi^{2}}$, the periodicity condition is imposed on the fluctuation on $S^{2}$ and the overall factor $\Sigma_{s}$ is divided. In this way, $S^{3}$ is realized in terms of the three matrices $X_{1}, X_{2}, X_{3}$.

In sections $3-5$, we generalize the results in this section. We set $\mu=1$ and set all other dimensionful parameters to a certain constant value.

\section{Dimensional reduction on a principal bundle}

In this section, we provide the dimensional reduction of YM on a principal $G$ bundle to its base space. The case of principal $U(1)$ bundles was already given in [29]. Here we consider the case where $G$ is nonabelian.

First, we give a metric and a vielbein of a fiber bundle on which pure YM is defined. We consider a principal $G$-bundle $P$ on a manifold $M$. The base space $M$ has a covering $\mathcal{S}$, and the total space has a covering $\left\{\pi^{-1}(U) \mid U \in \mathcal{S}\right\} . \pi^{-1}(U)$ is diffeomorphic to $U \times G$ by the local trivialization. Thus it is parameterized by $z^{M}=\left(x^{\mu}, y^{m}\right)(\mu=1, \cdots, \operatorname{dim} M ; m=$ $1, \cdots, \operatorname{dim} G)$, where $x^{\mu}$ parameterize the local patch $U$ and $y^{m}$ parameterize an element of $G$. We assume that the connection of $P$ is expressed as

$$
\omega=g^{-1}(y) b(x) g(y)-i g^{-1}(y) d g(y) .
$$


where $g(y) \in G, b(x)=b_{\mu}^{a}(x) T^{a} d x^{\mu}$ and $T^{a}$ are the generators of the Lie group $G$.

The transition functions of a principal bundle act on fibers by left multiplication. If there is overlap between $U$ and $U^{\prime}$, the relation between fiber coodinates, $g(y)$ on $U$ and $g\left(y^{\prime}\right)$ on $U^{\prime}$, is given by

$$
g\left(y^{\prime}\right)=k(x) g(y)
$$

where $k(x) \in G$. In the overlapping region $U \cap U^{\prime}, b(x)$ must transform as

$$
b^{\prime}\left(x^{\prime}\right)=k(x) b(x) k^{-1}(x)+i d k(x) k^{-1}(x) .
$$

Indeed, by using (3.3), we can show

$$
\omega=g(y)^{-1} b(x) g(y)-i g(y)^{-1} d g(y)=g\left(y^{\prime}\right)^{-1} b^{\prime}\left(x^{\prime}\right) g\left(y^{\prime}\right)-i g\left(y^{\prime}\right)^{-1} d g\left(y^{\prime}\right)
$$

We assume that the total space is endowed with a metric that has the fibered structure determined by the connection (3.1) and the isometry. As shown in [43], such metric can be locally expressed as 3

$$
\begin{aligned}
d s^{2} & =G_{M N} d z^{M} d z^{N} \\
& =g_{\mu \nu}(x) d x^{\mu} d x^{\nu}+2 \operatorname{Tr} \omega^{2} \\
& =g_{\mu \nu}(x) d x^{\mu} d x^{\nu}+\left\{e_{m}^{a}(y) d y^{m}-b_{\mu}^{a}(x) d x^{\mu}\right\}^{2} .
\end{aligned}
$$

Here $g_{\mu \nu}$ is a metric on the base space and $e_{m}^{a}(y)(a=\operatorname{dim} M+1, \cdots, \operatorname{dim} P)$ are the components of the right invariant Maurer-Cartan 1-form of $G$, which is defined by

$$
d g(y) g(y)^{-1}=-i e_{m}^{a}(y) T^{a} d y^{m} .
$$

We have assumed that the coefficient of the second term in (3.5) is just $\delta_{a b}$ so that the resultant dimensionally reduced theory is simple, although it is allowed to take $y$ independent function $\xi_{a b}(x)$. The Maurer-Cartan 1-form satisfies the Maurer-Cartan equation

$$
d e^{a}-\frac{1}{2} f^{a b c} e^{b} \wedge e^{c}=0
$$

where $f^{a b c}$ is the structure constant of the Lie algebra of $G$, and is regarded as the vielbein of the Cartan-Killing metric on $G$ defined by

$$
h_{m n}(y) d y^{m} d y^{n} \equiv-2 \operatorname{Tr}\left(d g g^{-1}\right)^{2}
$$

\footnotetext{
${ }^{3}$ Throughout of this paper, we use the following normalizations for the traces: $\operatorname{Tr}\left(T^{a} T^{b}\right)=\frac{1}{2} \delta_{a b}$ for the structure group of the fiber bundle and $\operatorname{tr}\left(T^{a} T^{b}\right)=\delta_{a b}$ for the gauge group.
} 


$$
=e_{m}^{a}(y) e_{n}^{a}(y) d y^{m} d y^{n} .
$$

Note that $e_{m}^{a}(y)$ and $b(x)$ in the metric (3.5) are defined locally on $U$ and must be transformed from $U$ to $U^{\prime}$ : the transformation of $e_{m}^{a}(y)$ is determined by $(\underline{3.2})$ and an equality

$$
d g\left(y^{\prime}\right) g\left(y^{\prime}\right)^{-1}=-i e_{m}^{a}\left(y^{\prime}\right) T^{a} d y^{\prime m}
$$

while the transformation of $b(x)$ is given in (3.3). By introducing a vielbein on the base space, $e_{\mu}^{\alpha}(x)(\alpha=1, \cdots, \operatorname{dim} M)$, one can write a vielbein and its inverse on the total space as follows:

$$
E_{M}^{A}(z)=\left(\begin{array}{cc}
e_{\mu}^{\alpha}(x) & 0 \\
-b_{\mu}^{a}(x) & e_{m}^{a}(y)
\end{array}\right), \quad E_{A}^{M}(z)=\left(\begin{array}{cc}
e_{\alpha}^{\mu}(x) & 0 \\
e_{a}^{m}(y) b_{\alpha}^{a}(x) & e_{a}^{m}(y)
\end{array}\right)
$$

where $e_{\alpha}^{\mu}$ and $e_{a}^{m}$ are the inverse of $e_{\mu}^{\alpha}$ and $e_{m}^{a}$, respectively, and $b_{\alpha}^{a}(x) \equiv e_{\alpha}^{\mu}(x) b_{\mu}^{a}(x)$. The local Lorentz frame defined by (3.10) gives the vertical-horizontal decomposition of vectors and 1-forms on the total space. Namely, $\alpha=1, \cdots, \operatorname{dim} M$ correspond to the directions to those of the base space and $a=\operatorname{dim} M+1, \cdots, \operatorname{dim} P$ correspond of the fiber space. Again, we remark that these expressions are defined locally on $U$. From (3.3) and (3.9), we can obtain relationships of the vielbeins between on $U$ and on $U^{\prime}$ as

$$
\begin{aligned}
& E^{\prime \alpha}=E^{\alpha}, \\
& E^{\prime a}=\operatorname{Ad}(k)^{a b} E^{b} .
\end{aligned}
$$

where $\operatorname{Ad}(k)$ is the adjoint representation of $k(x)$. (2.8) is a counterpart of (3.5) , and (2.10) and (2.11) are a counterpart of (3.10).

We next consider a gauge theory on the total space and make a dimensional reduction of the fiber direction to obtain a gauge theory on the base space. We start with $U(N)$ YM on the total space:

$$
S_{P}=\frac{1}{g_{P}^{2}} \int d^{D} z \sqrt{G} \operatorname{tr}\left(\frac{1}{4} F_{M N} F^{M N}\right) .
$$

where $D=\operatorname{dim} P$ and $F_{M N}=\partial_{M} A_{N}-\partial_{N} A_{M}+i\left[A_{M}, A_{N}\right]$. In order to make the reduction, we perform the vertical-horizontal decompostion for the gauge field $A_{M}(z)$ and the derivatives $\partial_{M}$ according to (3.10). The gauge field is decomposed as

$$
A_{M}(z)=A_{\alpha}(z) E_{M}^{\alpha}(x)+A_{a}(z) E_{M}^{a}(z) .
$$

After the reduction, horizontal components $A_{\alpha}$ and vertical components $A_{a}$ of the gauge field will be naturally identified with the gauge field and the higgs fields on the base space, respectively. The field strength in the local Lorentz frame is rewritten as follows:

$$
F_{\alpha \beta}=\nabla_{\alpha}^{(M)} A_{\beta}-\nabla_{\beta}^{(M)} A_{\alpha}+i\left[A_{\alpha}, A_{\beta}\right]-b_{\alpha \beta}^{a} A_{a}+i b_{\alpha}^{a} \mathcal{L}_{a} A_{\beta}-i b_{\beta}^{a} \mathcal{L}_{a} A_{\alpha},
$$




$$
\begin{aligned}
& F_{\alpha a}=e_{\alpha}^{\mu} \partial_{\mu} A_{a}+i\left[A_{\alpha}, A_{b}\right]-f^{a b c} b_{\alpha}^{b} A_{c}-i \mathcal{L}_{a} A_{\alpha}+i b_{\alpha}^{b} \mathcal{L}_{b} A_{a}, \\
& F_{a b}=f^{a b c} A_{c}+i\left[A_{a}, A_{b}\right]+i \mathcal{L}_{a} A_{b}-i \mathcal{L}_{b} A_{a} .
\end{aligned}
$$

Here we have defined the following quantities:

$$
\begin{aligned}
b_{\alpha \beta}^{a} & \equiv e_{\alpha}^{\mu} e_{\beta}^{\nu}\left\{\partial_{\mu} b_{\nu}^{a}-\partial_{\nu} b_{\mu}^{a}-f^{a b c} b_{\mu}^{b} b_{\nu}^{c}\right\} \\
\nabla_{\alpha}^{(M)} A_{\beta} & \equiv e_{\alpha}^{\mu}\left(\partial_{\mu} A_{\beta}+\omega_{\mu \beta}{ }^{\gamma} A_{\gamma}\right) \\
\mathcal{L}_{a} & \equiv-i e_{a}^{m} \partial_{m}
\end{aligned}
$$

where $\omega$ is the spin connection on the base space defined by $e_{\mu}^{\alpha}$ and $\mathcal{L}_{a}$ are the right invariant Killing vectors on the total space, which represent the isometry. Note that our calculations have been performed on $U$ so far. When it is performed on $U^{\prime}$, the quantities on $U^{\prime}$ must be used. The transformation of $b_{\alpha}^{a}(x)$ between on $U$ and on $U^{\prime}$ is given by (3.3), so that that of $b_{\alpha \beta}^{a}(x)$ is given by

$$
b_{\alpha \beta}^{\prime a}\left(x^{\prime}\right)=\operatorname{Ad}(k)^{a b} b_{\alpha \beta}^{b}(x) .
$$

The gauge field with the local Lorentz index must be transformed according to (3.11) as

$$
\begin{aligned}
& A_{\alpha}^{\prime}=A_{\alpha}, \\
& A_{a}^{\prime}=\operatorname{Ad}(k)^{a b} A_{b} .
\end{aligned}
$$

In order to make the dimensional reduction, we relate the fields on the total space to those on the base space as

$$
\begin{aligned}
& A_{\alpha}=a_{\alpha}, \\
& A_{a}=\phi_{a},
\end{aligned}
$$

where $a_{\alpha}$ are the gauge field in the local Lorentz frame and $\phi_{a}$ are higgs fields on the base space. We assume the both sides in (3.18) are independent of $y^{m}$. Using subscript of curved space, we can write (3.18) equivalently as

$$
\begin{aligned}
& A_{\mu}=a_{\mu}-b_{\mu}^{a} \phi_{a}, \\
& A_{m}=e_{m}^{a} \phi_{a} .
\end{aligned}
$$

Here (3.18) and (3.19) are a generalization of (2.13) and (2.14), respectively. Substituting (3.14) and (3.18) into (3.12) and using $\sqrt{G}=\sqrt{g} \sqrt{h}$, we obtain YM-higgs on the base space:

$$
S_{M}=\frac{1}{g_{M}^{2}} \int d^{d} x \sqrt{g} \operatorname{tr}\left\{\frac{1}{4}\left(f_{\alpha \beta}-b_{\alpha \beta}^{a} \phi_{a}\right)^{2}+\frac{1}{2}\left(\nabla_{\alpha}^{(M)} \phi_{a}+i\left[a_{\alpha}, \phi_{a}\right]-f^{a b c} b_{\alpha}^{b} \phi_{c}\right)^{2}\right.
$$




$$
\left.+\frac{1}{4}\left(f^{a b c} \phi_{c}+i\left[\phi_{a}, \phi_{b}\right]\right)^{2}\right\}
$$

where $g_{M}^{2}=\left(\int d y \sqrt{h}\right)^{-1} g_{P}^{2}=\frac{1}{\operatorname{Vol}(G)} g_{P}^{2}, d=\operatorname{dim} M$ and $f_{\alpha \beta}=\nabla_{\alpha}^{(M)} a_{\beta}-\nabla_{\beta}^{(M)} a_{\alpha}+i\left[a_{\alpha}, a_{\beta}\right]$. Note that the connection in the fiber bundle can generate nontrivial mass terms of the higgs fields. This is reminiscent of the flux compactification in string theory.

\section{Extension of the matrix T-duality}

In this section, we extend the matrix T-duality on nontrivial $U(1)$ bundles developed in [29] to that on nontrivial $S U(2)$ bundles.

\subsection{Nontrivial vacua and transformation between patches}

As in the example in section 2, the theory on the base space (3.20) has monopolelike vacua, which are in general patch-dependent if the principal bundle we consider is nontrivial. Here we describe the vacua and their patch-dependence. We also consider how the fields of the theory are transformed from a patch to another. We examine, in particular, the transformation properties of fluctuations around the vacua.

It is seen from (3.20) that the condition for the vacua is given by

$$
\begin{aligned}
& f_{\alpha \beta}-b_{\alpha \beta}^{a} \phi_{a}=0, \\
& \nabla_{\alpha}^{(M)} \phi_{a}+i\left[a_{\alpha}, \phi_{a}\right]-f^{a b c} b_{\alpha}^{b} \phi_{c}=0, \\
& f^{a b c} \phi_{c}+i\left[\phi_{a}, \phi_{b}\right]=0 .
\end{aligned}
$$

They are satisfied by the following configurations:

$$
\begin{aligned}
& \hat{a}_{\alpha}(x)=b_{\alpha}^{a}(x) \hat{\phi}_{a}=b_{\alpha}^{a}(x) L_{a}, \\
& \hat{\phi}_{a}=L_{a},
\end{aligned}
$$

where $L_{a}$ are the generators of the Lie algebra of $G$ satisfying $\left[L_{a}, L_{b}\right]=i f^{a b c} L^{c}$ and generally reducible. Note that as mentioned in section $3.1, b^{a}(x)$ are generally patch-dependent quantities. The vacua are, therefore, also patch-dependent. From (3.3) and (4.2), we can read off the transformation properties for the vacua between patches:

$$
\begin{aligned}
& \hat{a}^{\prime}(x)=K(x) \hat{a}(x) K(x)^{-1}+i d K(x) K(x)^{-1}, \\
& \hat{\phi}_{a}^{\prime}=\operatorname{Ad}(k(x))_{a b} K(x) \hat{\phi}_{b} K(x)^{-1}=\hat{\phi}_{a},
\end{aligned}
$$

where $K(x)$ is obtained by replacing $T^{a}$ in $k(x)$ in (3.2) by $\hat{\phi}_{a}=L_{a}$. Note that this is the gauge transformation by $K(x)$ except for the rotation of $\hat{\phi}_{a}$ by $\operatorname{Ad}(k(x))$, which comes from (3.17). 
Let us consider the theory around the vacua (4.2) and decompose the fields into the backgrounds and fluctuations:

$$
\begin{aligned}
& a_{\alpha}(x)=\hat{a}_{\alpha}(x)+\tilde{a}_{\alpha}(x), \\
& \phi_{a}(x)=\hat{\phi}_{a}+\tilde{\phi}_{a}(x) .
\end{aligned}
$$

The fluctuations are transformed between patches as

$$
\begin{aligned}
\tilde{a}_{\alpha}^{\prime}(x) & =K(x) \tilde{a}_{\alpha}(x) K(x)^{-1} \\
\tilde{\phi}_{a}^{\prime}(x) & =\operatorname{Ad}(k(x))_{a b} K(x) \tilde{\phi}_{b}(x) K(x)^{-1}
\end{aligned}
$$

One can easily see that the action (3.20) is indeed invariant under the transformation (4.3) and (4.5).

\section{2 $G=U(1), S U(2)$}

In this subsection, we consider the case in which the fiber is $U(1)$ or $S U(2)$. In the case of $G=U(1)$, the matrix T-duality indeed works as shown in [29] and its typical example was given in section 2 . We extend the matrix T-duality to the case of $G=S U(2)$ by applying the fact described in section 2 that YM on $S^{3}$ is realized in the matrix model.

First, we review the matrix T-duality in the case of $G=U(1)$, which is a generalization of the relationship between YM on $S^{3}$ and YM-higgs on $S^{2}$ in section 2. In this case, the metric (3.5) reduces to the following form:

$$
d s^{2}=g_{\mu \nu}(x) d x^{\mu} d x^{\nu}+\left(d y-b_{\mu}(x) d x^{\mu}\right)^{2}
$$

where $y$ represents the fiber direction and $0 \leq y<2 \pi$. We put $\operatorname{dim} M=d$. (2.8) indeed takes the form of (4.6). YM-higgs on the base space obtained from YM on the total space is given as the $U(1)$ case of (3.20):

$$
S_{M}=\frac{1}{g_{M}^{2}} \int d^{d} x \sqrt{g} \operatorname{tr}\left\{\frac{1}{4}\left(f_{\alpha \beta}-b_{\alpha \beta} \phi\right)^{2}+\frac{1}{2}\left(\nabla_{\alpha}^{(M)} \phi+i\left[a_{\alpha}, \phi\right]\right)^{2}\right\} .
$$

(2.15) is a special case of (4.7). We show that we obtain the $U(N)$ YM on the total space from the $U(N \times \infty)$ YM-higgs on the base space through the following procedure: we choose a certain background of the $U(N \times \infty)$ YM-higgs on the base space, expand the theory around the background and impose a periodicity condition.

Note, first, that a general background of (4.7) is given by

$$
\hat{a}_{\alpha}=b_{\alpha} \hat{\phi}
$$




$$
\hat{\phi}=-\operatorname{diag}(\cdots, \underbrace{n_{s-1}, \cdots, n_{s-1}}_{N_{s-1}}, \underbrace{n_{s}, \cdots, n_{s}}_{N_{s}}, \underbrace{n_{s+1}, \cdots, n_{s+1}}_{N_{s+1}}, \cdots),
$$

which is a counterpart of (2.22). We decompose the fields into the backgrounds and the fluctuations as

$$
\begin{aligned}
a_{\alpha} & \rightarrow \hat{a}_{\alpha}+a_{\alpha}, \\
\phi & \rightarrow \hat{\phi}+\phi .
\end{aligned}
$$

In particular, we take the following background: $s$ running from $-\infty$ to $\infty, n_{s}=s$ and $N_{s}=N$. We label the (off-diagonal) blocks by $(s, t)$ and impose the periodicity (orbifolding) condition on the fluctuations as in (2.35):

$$
\begin{gathered}
a_{\alpha}^{(s+1, t+1)}=a_{\alpha}^{(s, t)} \equiv a_{\alpha}^{(s-t)} \\
\phi^{(s+1, t+1)}=\phi^{(s, t)} \equiv \phi^{(s-t)}
\end{gathered}
$$

The fluctuations are gauge-transformed from $U$ to $U^{\prime}$ as

$$
\begin{aligned}
& a_{\alpha}^{\prime(s-t)}=e^{-i(s-t) v(x)} a_{\alpha}^{(s-t)}, \\
& \phi^{\prime(s-t)}=e^{-i(s-t) v(x)} \phi^{(s-t)}
\end{aligned}
$$

where $e^{-i v}$ is a transition function; $e^{-i y^{\prime}}=e^{-i v(x)} e^{-i y}$. (2.36) is a special case of (4.11). We make the Fourier transformation for the fluctuations on each patch to construct the gauge field on the total space:

$$
\begin{aligned}
A_{\alpha}(x, y) & =\sum_{w} a_{\alpha}^{(w)}(x) e^{-i w y} \\
A_{d+1}(x, y) & =\sum_{w} \phi^{(w)}(x) e^{-i w y} .
\end{aligned}
$$

We can see from (4.11) that the lefthand sides in the above equations are indeed invariant under the transformation between patches. Using (4.9) and (4.12), we can rewrite each term in (4.7) as

$$
\begin{aligned}
& \left(f_{\alpha \beta}-b_{\alpha \beta} \phi\right)^{(s, t)} \\
& \rightarrow\left(\nabla_{\alpha}^{(M)} a_{\beta}-\nabla_{\beta}^{(M)} a_{\alpha}+i\left[\hat{a}_{\alpha}, a_{\beta}\right]+i\left[a_{\alpha}, \hat{a}_{\beta}\right]+i\left[a_{\alpha}, a_{\beta}\right]-b_{\alpha \beta} \phi\right)^{(s, t)} \\
& =\left(\nabla_{\alpha}^{(M)} a_{\beta}^{(s-t)}-\nabla_{\beta}^{(M)} a_{\alpha}^{(s-t)}+i\left[a_{\alpha}, a_{\beta}\right]^{(s-t)}-i(s-t) b_{\alpha} a_{\beta}^{(s-t)}+i(s-t) b_{\beta} a_{\alpha}^{(s-t)}-b_{\alpha \beta} \phi^{(s-t)}\right) \\
& =\frac{1}{2 \pi} \int d y\left(\nabla_{\alpha}^{(M)} A_{\beta}-\nabla_{\beta}^{(M)} A_{\alpha}+i\left[A_{\alpha}, A_{\beta}\right]-b_{\alpha \beta} A_{d+1}+b_{\alpha} \partial_{y} A_{\beta}-b_{\beta} \partial_{y} A_{\alpha}\right) e^{i(s-t) y}
\end{aligned}
$$




$$
\begin{aligned}
& =\frac{1}{2 \pi} \int d y F_{\alpha \beta} e^{i(s-t) y} \\
& \left(\nabla_{\alpha}^{(M)} \phi+i\left[a_{\alpha}, \phi\right]\right)^{(s, t)} \\
& \rightarrow\left(\nabla_{\alpha}^{(M)} \phi+i\left[\hat{a}_{\alpha}, \phi\right]+i\left[a_{\alpha}, \hat{\phi}\right]+i\left[a_{\alpha}, \phi\right]\right)^{(s, t)} \\
& =\nabla_{\alpha}^{(M)} \phi^{(s-t)}+i\left[a_{\alpha}, \phi\right]^{(s-t)}-i(s-t) b_{\alpha} \phi^{(s-t)}+i(s-t) a_{\alpha}^{(s-t)} \\
& =\frac{1}{2 \pi} \int d y\left(\nabla_{\alpha}^{(M)} A_{d+1}+i\left[A_{\alpha}, A_{d+1}\right]-\partial_{y} A_{\alpha}+b_{\alpha} \partial_{y} A_{d+1}\right) e^{i(s-t) y} \\
& =\frac{1}{2 \pi} \int d y F_{\alpha(d+1)} e^{i(s-t) y} .
\end{aligned}
$$

Then (4.7) becomes

$$
\begin{aligned}
& S_{M}= \frac{1}{g_{M}^{2}} \int d^{d} x \sqrt{g} \operatorname{tr}\left\{\frac{1}{4}\left(f_{\alpha \beta}-b_{\alpha \beta} \phi\right)^{2}+\frac{1}{2}\left(\nabla_{\alpha}^{(M)} \phi_{a}+i\left[a_{\alpha}, \phi\right]\right)^{2}\right\} \\
&=\frac{1}{g_{M}^{2}} \int d^{d} x \sqrt{g} \operatorname{tr}\left[\sum _ { s , t } \left\{\frac{1}{4}\left(f_{\alpha \beta}-b_{\alpha \beta} \phi\right)^{(s, t)}\left(f_{\alpha \beta}-b_{\alpha \beta} \phi\right)^{(t, s)}\right.\right. \\
&\left.\left.\quad+\frac{1}{2}\left(\nabla_{\alpha}^{(M)} \phi+i\left[a_{\alpha}, \phi\right]\right)^{(s, t)}\left(\nabla_{\alpha}^{(M)} \phi+i\left[a_{\alpha}, \phi\right]\right)^{(t, s)}\right\}\right] \\
& \rightarrow \frac{1}{g_{M}^{2}} \frac{1}{2 \pi} \sum_{w} \int d^{D} z \sqrt{G} \frac{1}{4} \operatorname{tr}\left(F_{A B} F_{A B}\right) .
\end{aligned}
$$

By dividing an overall factor $\sum_{w}$ in the last line in (4.14) to extract a single period, we obtain Yang-Mills theory on the total space.

Next we consider the case where fiber is $S U(2)$. In this case, YM-higgs on the base space takes the form

$$
\begin{aligned}
S_{M}=\frac{1}{g_{M}^{2}} \int & d^{d} x \sqrt{g} \operatorname{tr}\left\{\frac{1}{4}\left(f_{\alpha \beta}-b_{\alpha \beta}^{a} \phi_{a}\right)^{2}+\frac{1}{2}\left(\nabla_{\alpha}^{(M)} \phi_{a}+i\left[a_{\alpha}, \phi_{a}\right]-\epsilon^{a b c} b_{\alpha}^{b} \phi_{c}\right)^{2}\right. \\
& \left.+\frac{1}{4}\left(\epsilon^{a b c} \phi_{c}+i\left[\phi_{a}, \phi_{b}\right]\right)^{2}\right\} .
\end{aligned}
$$

We show that we can obtain the $U(N)$ YM on the total space of a nontrivial $S U(2)$-bundle from the YM with three higgs on its base space in a way similar to the case of $G=U(1)$.

The vacuum of YM-higgs is given by (4.2) with $L_{a}$ satisfying the $S U(2)$ algebra, $\left[L_{a}, L_{b}\right]=$ $i \epsilon_{a b c} L_{c}$, and $L_{a}$ generically take a reducible representation (2.25). We expand the fields around this background,

$$
a_{\alpha}(x) \rightarrow \hat{a}_{\alpha}(x)+a_{\alpha}(x),
$$




$$
\phi_{a}(x) \rightarrow \hat{\phi}_{a}+\phi_{a}(x)
$$

We label the (off-diagonal) blocks of the fluctuations by $(s, t)$, which is $\left(N_{s}\left(2 j_{s}+1\right)\right) \times$ $\left(N_{t}\left(2 j_{t}+1\right)\right)$ matrix, and expand them by the fuzzy spherical harmonics:

$$
\begin{aligned}
& a_{\alpha}^{(s, t)}(x)=\sum_{J=\left|j_{s}-j_{t}\right|}^{j_{s}+j_{t}} \sum_{m=-J}^{J} a_{\alpha, J m}^{(s, t)}(x) \otimes \hat{Y}_{J m\left(j_{s} j_{t}\right)}, \\
& \phi_{a}^{(s, t)}(x)=\sum_{J=\left|j_{s}-j_{t}\right|}^{j_{s}+j_{t}} \sum_{m=-J}^{J} \phi_{a, J m}^{(s, t)}(x) \otimes \hat{Y}_{J m\left(j_{s} j_{t}\right)} .
\end{aligned}
$$

We verify from (4.5), (4.17) and (A.26) that the modes are gauge-transformed from $U$ to $U^{\prime}$ as

$$
\begin{aligned}
& a_{\alpha, J m}^{(s, t)}(x)=\sum_{m^{\prime}}\left\langle J m\left|k^{[J]}\right| J m^{\prime}\right\rangle a_{\alpha, J m^{\prime}}^{(s, t)}(x), \\
& \phi_{a, J m}^{\prime(s, t)}(x)=\sum_{m^{\prime}} \operatorname{Ad}(k)_{a b}\left\langle J m\left|k^{[J]}\right| J m^{\prime}\right\rangle \phi_{b, J m^{\prime}}^{(s, t)}(x),
\end{aligned}
$$

where $k^{[J]}$ is the spin $J$ representation of $S U(2)$ for $k(x)$.

In what follows, we assume that as a background we set $2 j_{s}+1=N_{0}+s$ with $s$ running from $-T$ to $T$ in (2.25) and take the limit of $N_{0} \rightarrow \infty$ and $T \rightarrow \infty$ in order. For the modes, we impose the periodicity condition:

$$
\begin{aligned}
& a_{\alpha, J m}^{(s+1, t+1)}=a_{\alpha, J m}^{(s, t)} \equiv a_{\alpha, J m}^{\left(q_{s t}\right)}, \\
& \phi_{a, J m}^{(s+1, t+1)}=\phi_{a, J m}^{(s, t)} \equiv \phi_{a, J m}^{\left(q_{s t}\right)},
\end{aligned}
$$

where $q_{s t} \equiv \frac{s-t}{2}$. By using these modes and the spherical harmonics on $S^{3}$, we make Fourier transformation on each patch to construct the gauge field on the total space:

$$
\begin{aligned}
& A_{\alpha}(z)=\sum_{J m \tilde{m}} a_{\alpha, J m}^{(\tilde{m})}(x) Y_{J m \tilde{m}}(y), \\
& A_{a}(z)=\sum_{J m \tilde{m}} \phi_{a, J m}^{(\tilde{m})}(x) Y_{J m \tilde{m}}(y) .
\end{aligned}
$$

Its inverse is

$$
\begin{aligned}
& a_{\alpha, J m}^{(\tilde{m})}(x)=\int \frac{d \Omega_{3}}{2 \pi^{2}} A_{\alpha}(z) Y_{J m \tilde{m}}^{\dagger}(y), \\
& \phi_{a, J m}^{(\tilde{m})}(x)=\int \frac{d \Omega_{3}}{2 \pi^{2}} A_{a}(z) Y_{J m \tilde{m}}^{\dagger}(y) .
\end{aligned}
$$

From (4.18) and (A.8), it is verified that the lefthand sides in (4.20) are indeed transformed between patches as the gauge field on the total space (3.17). 
Using (4.17) and (4.21), we can obtain the following equalities:

$$
\begin{aligned}
{\left[L_{a}, a_{\alpha}(x)\right]^{(s, t)} } & =\int \frac{d \Omega_{3}}{2 \pi^{2}}\left(\mathcal{L}_{a} A_{\alpha}(z)\right) Y_{J p q_{s t}}^{\dagger} \otimes \hat{Y}_{J p\left(j_{s} j_{t}\right)}, \\
{\left[\phi_{a}, \phi_{b}\right]^{(s, t)} } & =\int \frac{d \Omega_{3}}{2 \pi^{2}}\left[A_{a}(z), A_{b}(z)\right] Y_{J m q_{s t}}^{\dagger}(y) \otimes \hat{Y}_{J m\left(j_{s} j_{t}\right)} .
\end{aligned}
$$

The derivation of the above equalities is given in appendix B. Substituting these into (4.15), we obtain

$$
\begin{aligned}
S_{M}=\frac{1}{g_{M}^{2}} \int & d^{d} x \sqrt{g} \operatorname{tr}\left\{\frac{1}{4}\left(f_{\alpha \beta}-b_{\alpha \beta}^{a} \phi_{a}\right)^{2}+\frac{1}{2}\left(\nabla_{\alpha}^{(M)} \phi_{a}+i\left[a_{\alpha}, \phi_{a}\right]-\epsilon^{a b c} b_{\alpha}^{b} \phi_{c}\right)^{2}\right. \\
& \left.+\frac{1}{4}\left(\epsilon^{a b c} \phi_{c}+i\left[\phi_{a}, \phi_{b}\right]\right)^{2}\right\} \\
=\frac{1}{g_{M}^{2}} \int & d^{d} x \sqrt{g} \operatorname{tr}\left[\sum _ { s , t } \left\{\frac{1}{4}\left(f_{\alpha \beta}-b_{\alpha \beta}^{a} \phi_{a}\right)^{(s, t)}\left(f_{\alpha \beta}-b_{\alpha \beta}^{a} \phi_{a}\right)^{(t, s)}\right.\right. \\
& +\frac{1}{2}\left(\nabla_{\alpha}^{(M)} \phi_{a}+i\left[a_{\alpha}, \phi_{a}\right]-\epsilon^{a b c} b_{\alpha}^{b} \phi_{c}\right)^{(s, t)}\left(\nabla_{\alpha}^{(M)} \phi_{a}+i\left[a_{\alpha}, \phi_{a}\right]-\epsilon^{a b c} b_{\alpha}^{b} \phi_{c}\right){ }^{(t, s)} \\
& \left.\left.+\frac{1}{4}\left(\epsilon^{a b c} \phi_{c}+i\left[\phi_{a}, \phi_{b}\right]\right)^{(s, t)}\left(\epsilon^{a b c} \phi_{c}+i\left[\phi_{a}, \phi_{b}\right]\right)^{(t, s)}\right\}\right] \\
\rightarrow \frac{1}{g_{M}^{2}} \frac{N_{0}}{2 \pi^{2}} \sum_{w} \int d^{D} z \sqrt{G} & \\
& \times \operatorname{tr}\left\{\frac{1}{4}\left(\nabla_{\alpha}^{(M)} A_{\beta}-\nabla_{\beta}^{(M)} A_{\alpha}+i\left[A_{\alpha}, A_{\beta}\right]-b_{\alpha \beta}^{a} A_{a}+i b_{\alpha}^{a} \mathcal{L}_{a} A_{\beta}-i b_{\beta}^{a} \mathcal{L}_{a} A_{\alpha}\right)^{2}\right. \\
& +\frac{1}{2}\left(\nabla_{\alpha}^{(M)} A_{a}+i\left[A_{\alpha}, A_{b}\right]-f^{a b c} b_{\alpha}^{b} A_{c}-i \mathcal{L}_{a} A_{\alpha}+i b_{\alpha}^{b} \mathcal{L}_{b} A_{a}\right)^{2} \\
& \left.+\frac{1}{4}\left(f^{a b c} A_{c}+i\left[A_{a}, A_{b}\right]+i \mathcal{L}_{a} A_{b}-i \mathcal{L}_{b} A_{a}\right)^{2}\right\} \\
\frac{1}{g_{M}^{2}} \frac{N_{0}}{2 \pi^{2}} & \sum_{w} \int d^{d+1} z \sqrt{G} \operatorname{tr}\left(\frac{1}{4} F_{A B} F_{A B}\right)
\end{aligned}
$$

By dividing an overall factor $\sum_{w}$ in the last line in (4.23) to extract a single period, we obtain Yang-Mills theory on the total space.

We can easily extend the above matrix T-duality to the case in which the fiber is $S U(2)^{k} \times$ $U(1)^{l}$. As an example, we consider an $S U(2) \times U(1)$ bundle, $P$. Let $a, b, c$ in (3.20) run $0,1,2,3$ such that ' 0 ' corresponds to the $U(1)$ direction and ' $1,2,3$ ' correspond to the $S U(2)$ direction. We assign $i, j, k$ to the $S U(2)$ direction. We can consider YM-higgs on the $U(1)$ bundle on $M, M^{\prime}$, which is obtained by making the dimensional reduction of the $S U(2)$ fiber direction for $\mathrm{YM}$ on the $S U(2) \times U(1)$ bundle. We realize the theory around an $S U(2)$ 
multimonopole background of YM-higgs on $M^{\prime}$ by taking the following background in YMhiggs on $M(\underline{3.20}$ ) and imposing the periodicity condition to the fluctuations:

$$
\begin{aligned}
& \hat{\phi}_{0}=-\frac{1}{R} \operatorname{diag}(\cdots, t-1, t, t+1, \cdots) \otimes 1_{\hat{M}}+b_{0}^{i} \hat{\phi}_{i} \\
& \hat{\phi}_{i}=1_{\infty} \otimes\left(L_{i} \text { in }(2.25)\right) \\
& \hat{a}_{\alpha}=b_{\alpha}^{a} \hat{\phi}_{a}
\end{aligned}
$$

where $b_{\alpha}^{0}$ represents the $U(1)$ monopole and $b_{\alpha}^{i}$ represents the $\mathrm{SU}(2)$ monopole. $R$ is a certain constant depending on the fiber structure. By setting $2 j_{s}+1=N_{0}+s$ with $s$ running from $-T$ to $T$, taking the limit of $N_{0} \rightarrow \infty$ and $T \rightarrow \infty$ in order and imposing the periodicity condition to the fluctuations again, we realize YM on $P$ in YM-higgs on $M$. In a similar way, we can realize YM on an $S U(2)^{k} \times U(1)^{l}$ in YM-higgs on its base space.

\subsection{Example: $S^{7} \rightarrow S^{4}$}

We present an example of our findings in the previous subsection: we consider $S^{7}$ with radius 2 and regard it as $S U(2) \cong S^{3}$ Hopf bundle on $S^{4}$ with radius 1 .

In order to describe $S^{7}$ as $S U(2)$ bundle on $S^{4}$, it is convenient to introduce the quaternion $H$ (see for example [44-46]). The quaternion algebra is defined by

$$
\begin{aligned}
& \boldsymbol{i}^{2}=\boldsymbol{j}^{2}=\boldsymbol{k}^{2}=-1, \quad \boldsymbol{i} \boldsymbol{j}=-\boldsymbol{j} \boldsymbol{i}=\boldsymbol{k}, \\
& \boldsymbol{j} \boldsymbol{k}=-\boldsymbol{k} \boldsymbol{j}=\boldsymbol{i}, \quad \boldsymbol{k} \boldsymbol{i}=-\boldsymbol{i} \boldsymbol{k}=\boldsymbol{j} .
\end{aligned}
$$

An arbitrary element of $H$ is written as

$$
q=a+b \boldsymbol{i}+c \boldsymbol{j}+d \boldsymbol{k} .
$$

where $a, b, c, d \in R$. Its conjugation $q^{*}$ is defined by

$$
q^{*} \equiv a-b \boldsymbol{i}-c \boldsymbol{j}-d \boldsymbol{k}
$$

The absolute value is given by

$$
|q| \equiv \sqrt{q^{*} q}=\sqrt{a^{2}+b^{2}+c^{2}+d^{2}} \geq 0 .
$$

$S^{7}$ with radius 2 is expressed by using quaternions as follows:

$$
\left\{\left.\left(q_{1}, q_{2}\right) \in H^{2}|| q_{1}\right|^{2}+\left|q_{2}\right|^{2}=4\right\} .
$$

The Hopf map $\pi: S^{7} \rightarrow S^{4}$ is defined by

$$
\pi:\left(q_{1}, q_{2}\right) \rightarrow\left[\left(q_{1}, q_{2}\right)\right] \equiv\left\{\left(q_{1}, q_{2}\right) q \mid q \in H \backslash\{0\}\right\}
$$


In order to introduce local coordinates one needs to divide $S^{4}$ in two patches: $U_{1}\left(q_{1} \neq 0\right)$ and $U_{2}\left(q_{2} \neq 0\right)$. The local trivialization is given on each patch by

$$
\begin{aligned}
& \pi^{-1}\left(U_{1}\right) \ni\left(q_{1}, q_{2}\right) \rightarrow\left(q_{2} q_{1}^{-1}, q_{1}\left|q_{1}\right|^{-1}\right) \in U_{1} \times S U(2), \\
& \pi^{-1}\left(U_{2}\right) \ni\left(q_{1}, q_{2}\right) \rightarrow\left(q_{1} q_{2}^{-1}, q_{2}\left|q_{2}\right|^{-1}\right) \in U_{2} \times S U(2) .
\end{aligned}
$$

We parameterize $\left(q_{1}, q_{2}\right)$ by using a matrix representation of quaternions as

$$
\begin{aligned}
& q_{1}=2 \cos \frac{\chi}{2} \lambda, \\
& q_{2}=2 \sin \frac{\chi}{2} \kappa \lambda .
\end{aligned}
$$

where $\kappa, \lambda \in S U(2)$ are defined by using Pauli matrices $\sigma^{a}(a=1,2,3)$ as

$$
\begin{aligned}
& \kappa=e^{i \eta \frac{\sigma^{3}}{2}} e^{i \xi \frac{\sigma^{2}}{2}} e^{i \zeta \frac{\sigma^{3}}{2}}, \\
& \lambda=e^{-i \psi \frac{\sigma^{3}}{2}} e^{-i \theta \frac{\sigma^{2}}{2}} e^{-i \phi \frac{\sigma^{3}}{2}} .
\end{aligned}
$$

The ranges of variables in the above equations are

$$
\begin{aligned}
& 0 \leq \chi \leq \pi \\
& 0 \leq \xi \leq \pi, 0 \leq \eta<2 \pi, 0 \leq \zeta<4 \pi \\
& 0 \leq \theta \leq \pi, 0 \leq \phi<2 \pi, 0 \leq \psi<4 \pi
\end{aligned}
$$

In particular, $|\lambda|^{2}=\operatorname{det} \lambda=1$ and $|\kappa|^{2}=\operatorname{det} \kappa=1$ hold. One can easily see from (4.32) and (4.33) that on $U_{1}$ the fiber space $S U(2)$ is described by $\lambda$ while on $U_{2}$ that is described by $\lambda^{\prime} \equiv \kappa \lambda$. In the following, we restrict ourselves to the region $U_{1}$. We denote sets of coordinates as $x^{\mu}=(\chi, \xi, \eta, \zeta)=\left(\chi, x^{\bar{\mu}}\right)$ and $y^{m}=(\theta, \phi, \psi) \cdot x^{\mu}$ are coordinates of $S^{4}, x^{\bar{\mu}}$ are those of $S^{3}$ inside of $S^{4}$ and $y^{m}$ are those of $S U(2)$ of fiber. In order to describe a metric of $S^{7}$ explicitly, we introduce the Maurer-Cartan 1-forms for $\kappa$ and $\lambda$

$$
\begin{aligned}
& \kappa(\bar{x})^{\dagger} d \kappa(\bar{x})=i \bar{e}_{\bar{\mu}}^{a}(\bar{x}) \frac{\sigma^{a}}{2} d x^{\bar{\mu}}, \\
& d \lambda(y) \lambda(y)^{\dagger}=-i e_{m}^{a}(y) \frac{\sigma^{a}}{2} d y^{m},
\end{aligned}
$$

where $\bar{x}$ represents the set of $\left\{x^{\bar{\mu}}\right\}$. Then we define the metric of $S^{7}$ as

$$
d s_{S^{7}}^{2}=\operatorname{det}\left(d q_{1}\right)+\operatorname{det}\left(d q_{2}\right),
$$

which is evaluated as

$$
d s_{S^{7}}^{2}=\left(d \chi^{2}+\frac{1}{4} \sin ^{2} \chi \bar{e}_{\bar{\mu}}^{a}(\bar{x}) \bar{e}_{\bar{\nu}}^{a}(\bar{x}) d x^{\bar{\mu}} d x^{\bar{\nu}}\right)+\left(e_{m}^{a}(y) d y^{m}-\sin ^{2} \frac{\chi}{2} \bar{e}_{\bar{\mu}}^{a}(\bar{x}) d x^{\bar{\mu}}\right)^{2} .
$$


In the above expression, the first term represents the metric of the base space $S^{4}$ and the second one represents that of the fiber space $S U(2)$ locally. Note that $\frac{1}{4} \bar{e}_{\bar{\mu}}^{a} \bar{e}_{\bar{\nu}}^{a}$ and $\frac{1}{4} e_{m}^{a} e_{n}^{a}$ are a metric of $S^{3}$ with radius 1 . From (4.38) one can read off the vielbein on $S^{4}$ and the local connections of the fiber bundle as

$$
\begin{gathered}
e^{\alpha}{ }_{\mu}(x)=\left(\begin{array}{cc}
1 & 0 \\
0 & \frac{1}{2} \sin \chi \bar{e}^{a}{ }_{\bar{\mu}}(\bar{x})
\end{array}\right), \quad e_{\alpha}^{\mu}(x)=\left(\begin{array}{cc}
1 & 0 \\
0 & \frac{2}{\sin \chi} \bar{e}^{\bar{\mu}}{ }_{a}(\bar{x})
\end{array}\right) \\
b_{\chi}^{a}(x)=0, \quad b_{\bar{\mu}}^{a}(x)=\tan \frac{\chi}{2} e_{\bar{\mu}}^{a}(x) \\
b_{\chi \bar{\nu}}^{a}(x)=e_{\bar{\nu}}^{a}(x), \quad b_{\bar{\mu} \bar{\nu}}^{a}(x)=f^{a b c} e_{\bar{\mu}}^{b}(x) e_{\bar{\nu}}^{c}(x) .
\end{gathered}
$$

As noted before, when we move to the other region, $U_{2}$, we must change $\lambda$ to $\lambda^{\prime} \equiv \kappa \lambda$. Then, one can easily find that the local connections change to

$$
\begin{aligned}
& {b_{\chi}^{\prime}}_{\chi}^{a}(x)=0, \quad{b^{\prime}}_{\bar{\mu}}^{a}(x)=-\cot \frac{\chi}{2} \operatorname{Ad}(\kappa)^{a b} e_{\bar{\mu}}^{b}(x), \\
& b_{\chi \bar{\nu}}^{a}(x)=\operatorname{Ad}(\kappa)^{a b} b_{\chi \bar{\nu}}^{b}(x), \quad b_{\bar{\mu} \bar{\nu}}^{a}(x)=\operatorname{Ad}(\kappa)^{a b} b_{\bar{\mu} \bar{\nu}}^{b}(x) .
\end{aligned}
$$

This transformation property is consistent with (3.3). The vacua of (4.15) are given by (4.2), (2.25), (4.39) and (4.40) on each patch. $b_{\mu}^{a}$ and ${b^{\prime}}_{\mu}^{a}$ are known as the gauge field of the Yang monopole [47].

By applying the arguments in the previous subsection, we can show that YM on $S^{7}$ is equivalent to the theory around the multi Yang monopole background of YM-higgs on $S^{4}$ with the periodicity imposed.

\section{Gauge theories on $S U(n+1)(/ H)$ and matrix model}

In this section, we reveal various relations among gauge theories on $S U(n+1)$ and $S U(n+$ $1) / H$, where $H$ is $S U(n)$ or $S U(n) \times U(1)$ or $S U(n+1)$ which is a subgroup of $S U(n+1)$. Note that $S U(n+1) / S U(n) \simeq S^{2 n+1}$ and $S U(n+1) /(S U(n) \times U(1)) \simeq C P^{n}$ and for $H=S U(n+1)$ the corresponding gauge theory reduces to a matrix model. First, we develop a general formalism of a dimensional reduction by which one can obtain YM-higgs on $\tilde{G} / H$ from YM on $\tilde{G}$, where $\tilde{G}$ is an arbitrary group manifold. Applying this formalism to the case of $\tilde{G}=S U(n+1)$, we obtain YM-higgs on $S^{2 n+1}$ and on $C P^{n}$ and the matrix model. Next, by using the facts explained in appendix E, we show that the YM-higgs on $C P^{n}$ in the most general $U(1)$ monopole background is obtained by taking the commutative limit of the theory around a certain background of the matrix model. We have found the correct form of the YM-higgs type action of such theory on $C P^{n}$. Third, by using the extended matrix T-duality of the $U(1)$ case reviewed in section 4, we show that YM-higgs on $S^{2 n+1}$ is equivalent to the theory around a certain background of YM-higgs on $C P^{n}$ with the 
orbifolding condition imposed. Combining these two facts, we also show that YM-higgs on $S^{2 n+1}$ is realized as the theory around an appropriate background of the matrix model with the orbifolding condition imposed. Finally, by using the results in section 4, we show that YM on $S U(n+1)$ is realized in YM-higgs on $S U(N+1) /\left(S U(2)^{k} \times U(1)^{l}\right)$. In particular, it follows that YM on $S U(3)$ is realized in YM-higgs on $S^{5}$ and on $C P^{2}$.

\subsection{Dimensional reduction of YM theory on a group manifold}

In this subsection, we restrict ourselves to the case in which the total space $P$ is itself a group manifold $\tilde{G}$. In this case, we can take the Maurer-Cartan basis and rewrite the YM action on $\tilde{G}$ in such a way that the relation between YM on the total space and YM-higgs on the base space becomes more manifest. In terms of this expression of the YM action, we can easily perform the dimensional reduction to obtain the YM-higgs theory on a coset space $\tilde{G} / H$, where $H$ is a subgroup of $\tilde{G}$. Some conventions on the group manifold $\tilde{G}$ and the coset space $\tilde{G} / H$ are summarized in appendix C.

Let us consider pure YM on $\tilde{G}$. In the Maurer-Cartan basis, the gauge potential is written as $A=X_{A} E^{A}$ where $E^{A}$ are the right invariant 1-forms on $\tilde{G}$ which are defined in (C.2). In this basis, the field strength is written as

$$
\begin{aligned}
F & =d A+i A \wedge A \\
& =\frac{1}{2}\left(f_{A B C} X_{C}+i \mathcal{L}_{A} X_{B}-i \mathcal{L}_{B} X_{A}+i\left[X_{A}, X_{B}\right]\right) E^{A} \wedge E^{B},
\end{aligned}
$$

where we have used the Maurer-Cartan equation (C.3) and $\mathcal{L}_{\mathcal{A}}$ are the right invariant Killing vectors on $\tilde{G}$ which are defined in (C.9). This is a counterpart of (2.20). Then, the original YM action on $\tilde{G}$ is rewritten as follows:

$$
\frac{1}{g_{\tilde{G}}^{2}} \int \operatorname{tr}\left(\frac{1}{2} F \wedge * F\right)=\frac{1}{g_{\tilde{G}}^{2}} \int d^{D} z \sqrt{G} \operatorname{tr}\left\{\frac{1}{4}\left(f_{A B C} X_{C}+i \mathcal{L}_{A} X_{B}-i \mathcal{L}_{B} X_{A}+i\left[X_{A}, X_{B}\right]\right)^{2}\right\},
$$

where $D=\operatorname{dim}(\tilde{G}), G=\operatorname{det} G_{M N}$ and $G_{M N}$ is the metric on $\tilde{G}$. Note that the gauge transformation in this basis is given by

$$
X_{A} \rightarrow U X_{A} U^{-1}-\mathcal{L}_{A} U U^{-1} .
$$

As explained in appendix $\mathrm{C}$, if one drops the derivatives along the fiber direction in $\mathcal{L}_{A}$, these operators are reduced to the $L_{A}$ which are the Killing vectors on $\tilde{G} / H$ defined in (C.12). By dropping the derivatives along the fiber direction in $\mathcal{L}_{A}$ in (5.2), therefore, we can obtain the theory on $\tilde{G} / H$,

$\frac{1}{g_{\tilde{G}}^{2}} \int \operatorname{tr}\left(\frac{1}{2} F \wedge * F\right) \rightarrow \frac{1}{g_{\tilde{G} / H}^{2}} \int d^{d} x \sqrt{g} \operatorname{tr}\left\{\frac{1}{4}\left(f_{A B C} X_{C}+i L_{A} X_{B}-i L_{B} X_{A}+i\left[X_{A}, X_{B}\right]\right)^{2}\right\}$, 
where $g_{\tilde{G} / H}^{2}=g_{\tilde{G}}^{2} / \operatorname{Vol}(H), d=\operatorname{dim} \tilde{G} / H, g=\operatorname{det} g_{\mu \nu}$ and $g_{\mu \nu}$ is the metric on $\tilde{G} / H$. This is a counterpart of (2.18).

The action (5.4) is also rewritten into the YM-higgs form which was obtained in section 3. The relation between the fields $X_{A}$ and the gauge and higgs fields on $\tilde{G} / H$ is given as follows. We introduce the orthogonal vectors to $L_{A}$ as

$$
N_{A}^{a}=\operatorname{Ad}(L(x))_{A}^{a},
$$

where $L(x)$ is a representative element of $\tilde{G} / H$ which is defined in (C.1), and Ad represents the adjoint action: $g T^{A} g^{-1}=T^{B} \operatorname{Ad}(g)_{B A}$. One can show the orthonormality conditions,

$$
L_{A}^{\mu} L_{A}^{\nu}=-g^{\mu \nu}, \quad N_{A}^{a} N_{A}^{b}=\delta^{a b}, \quad L_{A}^{\mu} N_{A}^{a}=0,
$$

where $g^{\mu \nu}$ is the inverse of the metric on $\tilde{G} / H$. Furthermore, the following equalities hold:

$$
\begin{aligned}
& L_{A}^{\mu} \partial_{\mu} N_{B}^{a}-L_{B}^{\mu} \partial_{\mu} N_{A}^{a}=-2 i L_{A}^{\mu} L_{B}^{\nu} b_{\mu \nu}^{a}-f_{a b c}\left(L_{A}^{\mu} N_{B}^{b}-L_{B}^{\mu} N_{A}^{b}\right) b_{\mu}^{c}, \\
& f_{A B C} N_{C}^{a}-f_{a b c} N_{A}^{b} N_{B}^{c}+L_{A}^{\mu} L_{B}^{\nu} b_{\mu \nu}^{a}=0 .
\end{aligned}
$$

We decompose $X_{A}$ into the gauge and higgs fields in terms of $L_{A}^{\mu}$ and $N_{A}^{a}$ as follows [33]:

$$
X_{A}=i L_{A}^{\mu} a_{\mu}+N_{A}^{a} \phi_{a}
$$

This is a generalization of (2.16). Then, each term in the action (5.4) is rewritten as

$$
\begin{aligned}
f_{A B C} X_{C}= & i f_{A B C} L_{C}^{\mu} a_{\mu}-L_{A}^{\mu} L_{B}^{\nu} b_{\mu \nu}^{a} \phi_{a}+f_{a b c} N_{A}^{b} N_{B}^{c} \phi_{a} \\
i L_{A} X_{B}-i L_{B} X_{A}= & -i f_{A B C} L_{C}^{\mu} a_{\mu}-L_{A}^{\mu} L_{B}^{\nu}\left(\partial_{\mu} a_{\nu}-\partial_{\nu} a_{\mu}-2 b_{\mu \nu}^{a} \phi_{a}\right) \\
& +i\left(L_{A}^{\mu} N_{B}^{a}-L_{B}^{\mu} N_{A}^{a}\right)\left(\partial_{\mu} \phi_{a}-f_{a b c} b_{\mu}^{b} \phi_{c}\right) \\
i\left[X_{A}, X_{B}\right]= & -i L_{A}^{\mu} L_{B}^{\nu}\left[a_{\mu}, a_{\nu}\right]-\left(L_{A}^{\mu} N_{B}^{a}-L_{B}^{\mu} N_{A}^{a}\right)\left[a_{\mu}, \phi_{a}\right]+i N_{A}^{a} N_{B}^{b}\left[\phi_{b}, \phi_{a}\right],(5 .
\end{aligned}
$$

where we have used (5.7). By substituting these equations into the action (5.4) and using (5.6), we indeed obtain the YM-higgs type action (3.20),

$$
\begin{array}{r}
S_{\tilde{G} / H}=\frac{1}{g_{\tilde{G} / H}^{2}} \int d^{d} x \sqrt{g} \operatorname{tr}\left\{\frac{1}{4}\left(f_{\mu \nu}-b_{\mu \nu}^{a} \phi_{a}\right)^{2}\right. \\
+\frac{1}{2}\left(D_{\mu} \phi_{a}-f_{a b c} b_{\mu}^{b} \phi_{c}\right)^{2} \\
\left.+\frac{1}{4}\left(f_{a b c} \phi_{c}+i\left[\phi_{a}, \phi_{b}\right]\right)^{2}\right\} .
\end{array}
$$

Finally, we consider the case in which $P=\tilde{G}$ and the base manifold is just a point. This is the special case of the above dimensional reduction in which $H$ equals $\tilde{G}$ itself. In this 
case, the theory on the base space is given by a zero-dimensional matrix model. Dropping all the derivatives in (5.2), we can easily make a dimensional reduction to the matrix model:

$$
\frac{1}{g_{\tilde{G}}^{2}} \int \operatorname{tr}\left(\frac{1}{2} F \wedge * F\right) \rightarrow \frac{1}{g_{m m}^{2}} \operatorname{tr}\left\{\frac{1}{4}\left(f_{A B C} X_{C}+i\left[X_{A}, X_{B}\right]\right)^{2}\right\}
$$

where $g_{m m}^{2}=g_{\tilde{G}}^{2} / \operatorname{Vol}(\tilde{G})$. This is a counterpart of (2.19). Of course, we can obtain the matrix model (5.11) also from the theory (5.4) on $\tilde{G} / H$ by dropping the derivatives $L_{A}$. If we regard the original YM on $\tilde{G}$ as YM on a principal $\tilde{G}$ bundle over a point, we obtain (5.11) as a special case of (3.20).

\subsection{Dimensional reduction of YM theory on $S U(n+1)$}

In this subsection, we derive the YM-higgs on $S^{2 n+1}$ and on $C P^{n}$ by applying the dimensional reduction discussed in the previous subsection. We also derive the 0-dimensional matrix model in which the YM-higgs on $S^{2 n+1}$ and on $C P^{n}$ will be realized.

Let us consider the group manifold $S U(n+1)$. We can apply the dimensional reduction developed in section 5.1 to the case of $P=\tilde{G}=S U(n+1)$ and obtain a theory on a coset space $S U(n+1) / H$, where $H$ is a subgroup of $S U(n+1)$. We begin with pure YM on the group manifold $S U(n+1)$ in the Maurer-Cartan basis,

$$
S_{S U(n+1)}=\frac{1}{g_{S U(n+1)}^{2}} \int d^{n(n+2)} z \sqrt{G} \operatorname{tr}\left\{\frac{1}{4}\left(f_{A B C} X_{C}+i \mathcal{L}_{A} X_{B}-i \mathcal{L}_{B} X_{A}+i\left[X_{A}, X_{B}\right]\right)^{2}\right\},
$$

where $f_{A B C}$ is the structure constant of $S U(n+1), G=\operatorname{det} G_{M N}$ and $G_{M N}$ is the CartanKilling metric on $\tilde{G}$ which is defined in (․6).

Let us consider the dimensional reduction of the above theory to a theory on $\tilde{G} / H$. If we take $H$ to be $S U(n)$, the coset space is given by $S U(n+1) / S U(n) \simeq S^{2 n+1}$. By applying the dimensional reduction (5.4) to YM on $S U(n+1)$, therefore, we obtain the YM-higgs theory on $S^{2 n+1}$,

$$
S_{S^{2 n+1}}=\frac{1}{g_{S^{2 n+1}}^{2}} \int d^{2 n+1} \tilde{x} \sqrt{\tilde{g}} \operatorname{tr}\left\{\frac{1}{4}\left(f_{A B C} X_{C}+i \tilde{L}_{A} X_{B}-i \tilde{L}_{B} X_{A}+i\left[X_{A}, X_{B}\right]\right)^{2}\right\},
$$

where $\tilde{g}$ represents the determinant of the metric on $S^{2 n+1}$, and $\tilde{L}_{A}$ 's are the Killing vectors on $S^{2 n+1}$. Note that $S^{2 n+1}$ that we consider here possesses only $S U(n+1)$ isometry which is smaller than $S O(2 n+2)$. In fact, this is not the ordinary round sphere but a squashed sphere. In the case of $n=2$, the metric of this squashed $S^{5}$ is explicitly given in appendix D. 
Next, we consider the case of $H=S U(n) \times U(1)$. In this case, the coset space is $S U(n+1) /(S U(n) \times U(1)) \simeq C P^{n}$. Then, we can obtain the theory on $C P^{n}$ from YM on $S U(n+1)$ through the dimensional reduction,

$$
S_{C P^{n}}=\frac{1}{g_{C P^{n}}^{2}} \int d^{2 n} x \sqrt{g} \operatorname{tr}\left\{\frac{1}{4}\left(f_{A B C} X_{C}+i L_{A} X_{B}-i L_{B} X_{A}+i\left[X_{A}, X_{B}\right]\right)^{2}\right\} .
$$

As in the case of $S^{2 n+1}, g=\operatorname{det} g_{\mu \nu}, g_{\mu \nu}$ and $L_{A}$ represent the metric and the Killing vectors on $C P^{n}$ respectively. The theory (5.14) can be obtained also from the theory (5.13) by dropping the derivative along the extra $U(1)$ fiber direction. We can also rewrite (5.13) and (5.14) into the YM-higgs type actions as in (5.10) by using the relation (5.8). For example, (5.14) is rewritten into (5.10) with $\mu, \nu=1, \cdots, 2 n$ and $a, b, c=0, \cdots, n^{2}-1$. Here, $a, b, c$ are indices of $S U(n) \times U(1)$ and $a=0$ corresponds to the $U(1)$ direction.

Finally, we consider the case in which $H$ is $S U(n+1)$ itself. In this case, the coset space is just a point. Then, we obtain the following matrix model by using (5.11):

$$
S_{m m}=\frac{1}{g_{m m}^{2}} \operatorname{tr}\left\{\frac{1}{4}\left(f_{A B C} X_{C}+i\left[X_{A}, X_{B}\right]\right)^{2}\right\}
$$

This theory is used to realize the theories (5.13) and (5.14) in the next subsection. For $n=1$, the dimensional reductions in this subsection are equivalent to those in section 2.

\subsection{Relations among gauge theories on $S U(n+1) / H$}

In this subsection, we show that the theory (5.14) in a monopole background can be realized by taking the commutative limit of the theory around a nontrivial background of (5.15). Combining this construction and the matrix T-duality, we also show that the theory (5.13) on $S^{2 n+1}$ can be realized as the theory around a certain background of the matrix model with the orbifolding condition imposed. Furthermore, we apply the extended matrix Tduality developed in section 4 to YM-higgs on $S U(n+1) /\left(S U(2)^{k} \times U(1)^{l}\right)$ and show that YM on $S U(n+1)$ is equivalent to the theory around a certain vacuum of YM-higgs on $S U(n+1) /\left(S U(2)^{k} \times U(1)^{l}\right)$ with the periodicity condition imposed. For $n=2$, we obtain YM on $S U(3)$ from YM-higgs on $S^{5}$ and on $C P^{2}$ through the extended matrix T-duality.

First, we review nontrivial backgrounds of the theory (5.14) on $C P^{n}$ and the matrix model (5.15). The theory on $C P^{n}$ has many nontrivial monopole vacua. In particular, we focus on the $U(1)$ monopole background. Recall that we have $n^{2}$ higgs fields $\phi_{a}$. In the $U(1)$ monopole background, only the higgs field along the $U(1)$ direction $\phi_{0}$ acquires its nonzero vacuum expectation value. In the gauge where $\phi_{0}$ is diagonal, the vacuum configurations of the $U(1)$ monopole with the gauge group $U(M)$ are given by

$$
\hat{a}_{\mu}=b_{\mu}^{0} \hat{\phi}_{0}
$$




$$
\begin{aligned}
& \hat{\phi}_{0}=-\frac{1}{\sqrt{2 n(n+1)}} \operatorname{diag}(\cdots, \underbrace{n_{s-1}, \cdots, n_{s-1}}_{N_{s-1}}, \underbrace{n_{s}, \cdots, n_{s}}_{N_{s}}, \underbrace{n_{s+1}, \cdots, n_{s+1}}_{N_{s+1}}, \cdots), \\
& \hat{\phi}_{a}=0, \quad(\text { for } a \neq 0) .
\end{aligned}
$$

Here, $\sum_{s} N_{s}=M$ and $n_{s}$ must be integers due to Dirac's quantization condition. Because of (5.8), the vacuum configurations of $X_{A}$ are equivalently given by

$$
\hat{X}_{A}=-\frac{i L_{A}^{\mu} b_{\mu}^{0}+N_{A}^{0}}{\sqrt{2 n(n+1)}} \operatorname{diag}(\cdots, \underbrace{n_{s-1}, \cdots, n_{s-1}}_{N_{s-1}}, \underbrace{n_{s}, \cdots, n_{s}}_{N_{s}}, \underbrace{n_{s+1}, \cdots, n_{s+1}}_{N_{s+1}}, \cdots) .
$$

The theory around the background (5.17) is obtained by expanding each block of the fields in (5.14) as $X_{A}^{(s, t)} \rightarrow \hat{X}_{A}^{(s, t)}+X_{A}^{(s, t)}$. Then, the following action is obtained,

$$
\begin{aligned}
\frac{1}{g_{C P^{n}}^{2}} \int d^{2 n} x \sqrt{g} \sum_{s, t} \operatorname{tr}\{ & \frac{1}{4}\left(f_{A B C} X_{C}^{(s, t)}+i L_{A}^{\left(q_{s t}\right)} X_{B}^{(s, t)}-i L_{B}^{\left(q_{s t}\right)} X_{A}^{(s, t)}+i\left[X_{A}, X_{B}\right]^{(s, t)}\right) \\
\times & \left.\left(f_{A B D} X_{D}^{(t, s)}+i L_{A}^{\left(q_{t s}\right)} X_{B}^{(t, s)}-i L_{B}^{\left(q_{t s}\right)} X_{A}^{(t, s)}+i\left[X_{A}, X_{B}\right]^{(t, s)}\right)\right\},
\end{aligned}
$$

where $q_{s t}=\frac{n_{s}-n_{t}}{2}$ and $L_{A}^{(q)}$ are the angular momentum operators in the presence of a monopole with the magnetic charge $q$, which take the form

$$
L_{A}^{(q)}=L_{A}+\frac{2 q}{\sqrt{2 n(n+1)}}\left(i L_{A}^{\mu} b_{\mu}^{0}+N_{A}^{0}\right)
$$

These operators are the generalization of (2.27) in the case of $S^{2}$.

The vacua of the theory (5.15) are determined by

$$
\left[X_{A}, X_{B}\right]=i f_{A B C} X_{C}
$$

In addition to the trivial solution $X_{A}=0$, there are nontrivial solutions which are given by the representation matrices of the $S U(n+1)$ generators,

$$
\hat{X}_{A}=\hat{L}_{A} .
$$

$\hat{L}_{A}$ are generally in a reducible representation. In order to construct a theory on $C P^{n}$ in a 
$U(1)$ monopole background, we consider the following representation:

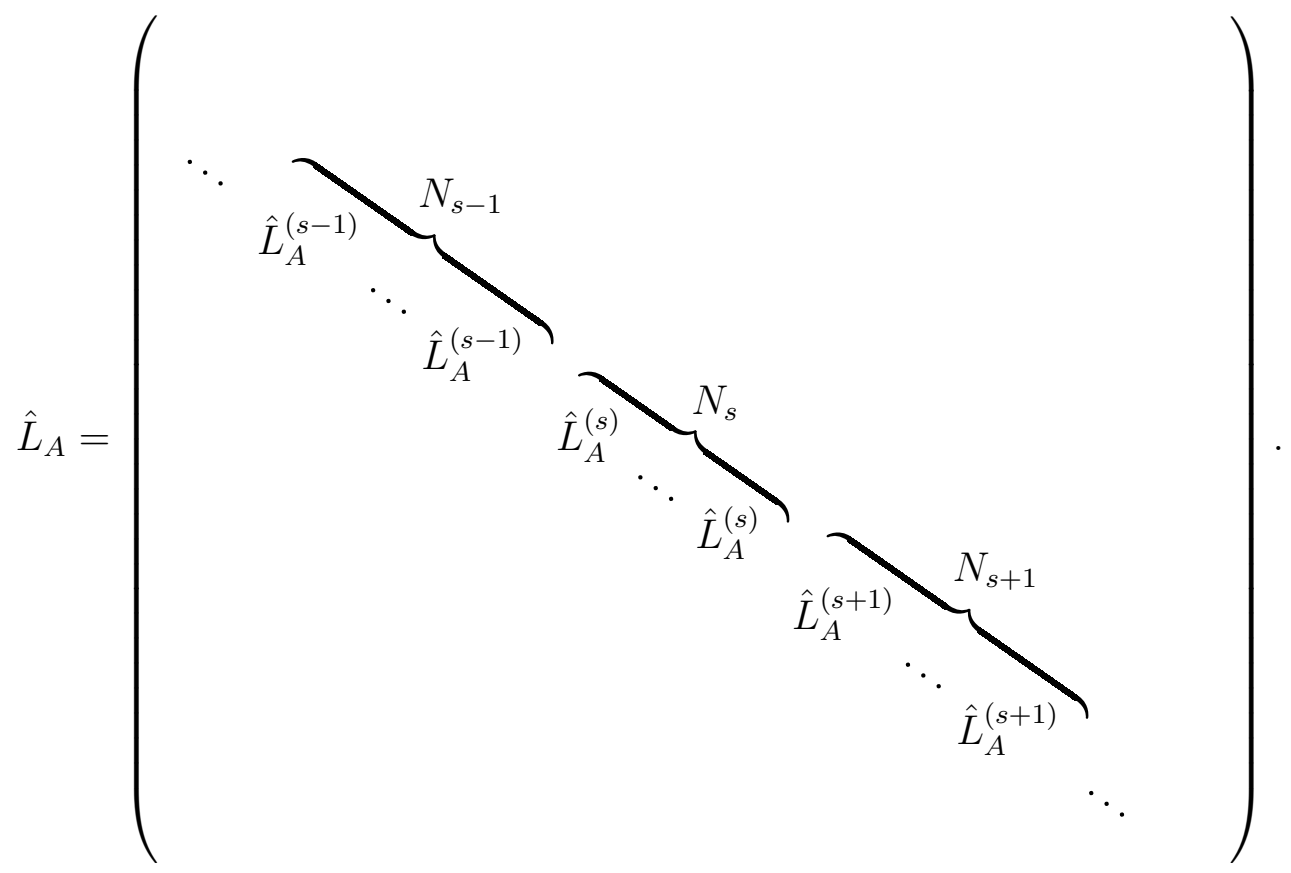

Here $\hat{L}_{A}^{(s)}$ are the abbreviations of $\hat{L}_{A}^{\left[\Lambda_{s}, 0, \cdots, 0\right]}$ which are the generators of $S U(n+1)$ in the irreducible representation specified by the Dynkin index of $S U(n+1),\left[\Lambda_{s}, 0, \cdots, 0\right]$. We consider the matrix model (5.15) around the background (5.22) by expanding the each block of the fields around the background $: X_{A}^{(s, t)} \rightarrow \hat{X}_{A}^{(s, t)}+X_{A}^{(s, t)}$. Then, the action takes the following form:

$$
\begin{aligned}
S_{m m}=\frac{1}{g_{m m}^{2}} \sum_{s, t} \operatorname{tr} & \left\{\frac{1}{4}\left(f_{A B C} X_{C}^{(s, t)}+i \hat{L}_{A} \circ X_{B}^{(s, t)}-i \hat{L}_{B} \circ X_{A}^{(s, t)}+i\left[X_{A}, X_{B}\right]^{(s, t)}\right)\right. \\
& \left.\times\left(f_{A B D} X_{D}^{(t, s)}+i \hat{L}_{A} \circ X_{B}^{(t, s)}-i \hat{L}_{B} \circ X_{A}^{(t, s)}+i\left[X_{A}, X_{B}\right]^{(t, s)}\right)\right\} .
\end{aligned}
$$

$\hat{L}_{A}$ o are defined as

$$
\hat{L}_{A} \circ X_{B}^{(s, t)} \equiv \hat{L}_{A}^{(s)} X_{B}^{(s, t)}-X_{B}^{(s, t)} \hat{L}_{A}^{(t)} .
$$

We show in the following that the theory (5.23) is equivalent to the theory (5.18) if we put $\Lambda_{s}=N_{0}+n_{s}$ and take $N_{0} \rightarrow \infty$ limit. In order to show this equivalence, we make a harmonic expansion $[31,32,35]$. As explained in appendix E, the $(s, t)$ blocks $X_{A}^{(s, t)}$ in the matrix model are expanded by the basis of rectangular matrices (E.21) as

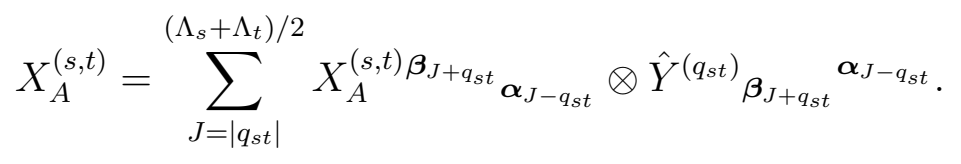


Then, the diagonal coherent map allows us to map the $(s, t)$ blocks to local sections of the monopole bundle on $C P^{n}$ with the charge $q_{s t}$,

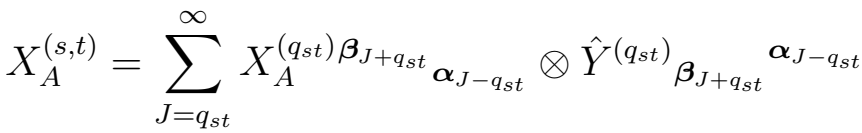

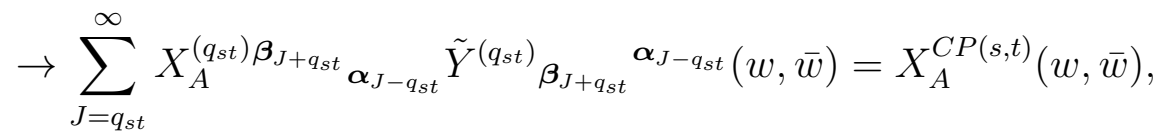

where we have taken the commutative limit $N_{0} \rightarrow \infty$ and $\tilde{Y}^{\left(q_{s t}\right)} \boldsymbol{\beta}_{J+q_{s t}}{ }^{\boldsymbol{\alpha}_{J-q_{s t}}}$ are the basis of local sections of the $U(1)$ monopole bundle on $C P^{n}$ which are defined in (E.26). Note that we have put the superscript $C P$ on the quantity in the righthand side of the above equation in order to emphasis that the $X_{A}^{C P(s, t)}$ are the fields on $C P^{n}$ appearing in (5.18). Similarly, $\hat{L}_{A}$ ○ is mapped to $L_{A}^{(q)}$ as shown in (E.31)

$$
\hat{L}_{A} \circ X_{B}^{(s, t)} \rightarrow L_{A}^{(q)} X_{B}^{C P(s, t)}(w, \bar{w})
$$

Using (5.26) and (5.27), we find that the matrix model (5.23) is equivalent to the theory (5.18) on $C P^{n}$ in the commutative limit $N_{0} \rightarrow \infty$.

Next, we show that the theory around a certain vacuum of $U(M=N \times \infty)$ YM-higgs on $C P^{n}$ with a periodicity condition imposed is equivalent to $U(N)$ YM-higgs on $S^{2 n+1}$. This statement is nothing but the matrix T-duality. As explained in section 4, therefore, we consider the appropriate vacuum which is given by (5.17) (or equivalently (5.16)) with $s$ running from $-\infty$ to $\infty, n_{s}=s$ and $N_{s}=N$. We expand the fields on $C P^{n}$ around the background as

$$
X_{A} \rightarrow \hat{X}_{A}+X_{A}
$$

and impose the periodicity (orbifolding) condition on the fluctuation,

$$
X_{A}^{(s+1, t+1)}=X_{A}^{(s, t)} \equiv X_{A}^{(s-t)} .
$$

Then, we define the gauge and higgs fields on $S^{2 n+1}$ by the Fourier transforms of the fluctuations on each local coordinate patch:

$$
X_{A}^{S}=\sum_{u} X_{A}^{C P(u)} e^{-i u y}
$$

where $y$ is a coordinate which parameterizes the fiber $(U(1))$ direction and satisfies $0 \leq \tau \leq$ $2 \pi$. Here, the superscripts $S$ and $C P$ indicate that $X_{A}^{S}$ and $X_{A}^{C P(w)}$ are the fields on $S^{2 n+1}$

\footnotetext{
${ }^{4}$ In [32], (5.26) and (5.27) are proven to the quadratic order in the fields for all $q$ and to all order for $q=0$. In this paper, we assume that these are also valid to all order for all $q$.
} 
and $C P^{n}$ respectively. We substitute (5.30) into (5.18) and divide an overall factor $\sum_{s}$ to extract a single period. Then, we obtain $U(N)$ YM-higgs on $S^{2 n+1}$ written in the basis of $X_{A}(\underline{5.13})$.

Combining the above matrix T-duality and the construction of (5.18) in terms of the matrix model, we find that the theory around (5.22) of the matrix model, where $s$ runs from $-\infty$ to $\infty$ and $\Lambda_{s}=N_{0}+s$, is equivalent to $U(N)$ YM-higgs on $S^{2 n+1}$ if we take the limit $N_{0} \rightarrow \infty$, impose the periodicity condition on the fluctuations, and finally divide the overall factor $\sum_{s}$.

Finally, it is straightforward to apply the extended matrix T-duality to $S U(2)^{k} \times U(1)^{l}$ bundle on $S U(n+1) /\left(S U(2)^{k} \times U(1)^{l}\right)$ and show that YM on $S U(n+1)$ is equivalent to the theory around a certain vacuum of YM-higgs on $S U(n+1) /\left(S U(2)^{k} \times U(1)^{l}\right)$ with the periodicity condition imposed.

\section{Interpretation as Buscher's T-duality}

In this section, let us see that the extended matrix T-duality of the $U(1)$ case, which was obtained in [29] and reviewed in section 4.2, is actually interpreted as the T-duality in Buscher's sense. We put $\operatorname{dim} M=p$. For $G=U(1)$, as in (4.6), the metric of the total space is given by

$$
d s^{2}=G_{M N} d z^{M} d z^{N}=g_{\mu \nu} d x^{\mu} d x^{\nu}+\left(d y-b_{\mu} d x^{\mu}\right)^{2}
$$

where $M, N=1, \cdots, p+1$ and $\mu, \nu=1, \cdots, p$. We assume that the other fields such as the antisymmetric fields and the dilaton field are trivial. Then, YM on the total space is viewed as the low energy effective theory for the $\mathrm{D} p$-branes wrapped on the total space 5 . We make the T-duality transformation for the fiber direction to obtain a new geometry [36]:

$$
\begin{aligned}
& d s^{2}=g_{\mu \nu} d x^{\mu} d x^{\nu}+d y^{2}, \\
& B^{\prime}{ }_{\mu \nu}=0, \quad B^{\prime}{ }_{\mu y}=-b_{\mu} .
\end{aligned}
$$

The $\mathrm{D} p$-branes should be transformed to the $\mathrm{D}(p-1)$-branes wrapped on the base space. The $\mathrm{D}(p-1)$-brane effective action on the new geometry (6.2) is given by

$$
S_{p-1}=\tau_{p-1} \int d^{p} \sigma e^{-\Phi} \sqrt{\operatorname{det}\left(\tilde{G}_{a b}+\tilde{B}_{a b}+2 \pi \alpha^{\prime} F_{a b}\right)}
$$

where $\sigma^{a}(a=1, \cdots, p)$ parameterize the world volume of the $\mathrm{D}(p-1)$-brane, and $\tilde{G}_{a b}$ and $\tilde{B}_{a b}$ are the pullback of (6.2) on the world volume which is defined through the embedding

\footnotetext{
${ }^{5}$ Here we ignore the transverse directions.
} 
of world volume $z^{M}(\sigma)$ as

$$
\begin{aligned}
\tilde{G}_{a b} & =\frac{\partial z^{M}}{\partial \sigma^{a}} \frac{\partial z^{N}}{\partial \sigma^{b}} G^{\prime}{ }_{M N}, \\
\tilde{B}_{a b} & =\frac{\partial z^{M}}{\partial \sigma^{a}} \frac{\partial z^{N}}{\partial \sigma^{b}} B^{\prime}{ }_{M N} .
\end{aligned}
$$

In the static gauge $x^{\mu}(\sigma)=\sigma^{\mu}$ and $z^{y}(\sigma)=2 \pi \alpha^{\prime} \phi$, (6.3) reduces to

$$
S_{p-1}=\tau_{p-1} \int d^{p} x \sqrt{\operatorname{det}\left(g_{\mu \nu}+\left(2 \pi \alpha^{\prime}\right)^{2} \partial_{\mu} \phi \partial_{\nu} \phi+2 \pi \alpha^{\prime}\left(F_{\mu \nu}+\partial_{\mu} \phi b_{\nu}-\partial_{\nu} \phi b_{\mu}\right)\right)} .
$$

Up to $\mathcal{O}\left(\alpha^{\prime 3}\right)$, this equals

$$
\frac{1}{g_{Y M}^{2}} \int d^{p} x \sqrt{g}\left(\frac{1}{4}\left(F_{\alpha \beta}+\nabla_{\alpha} \phi b_{\beta}-\nabla_{\beta} \phi b_{\alpha}\right)^{2}+\frac{1}{2}\left(\nabla_{\alpha} \phi\right)^{2}\right),
$$

where $g_{Y M}^{2}=\frac{1}{4 \pi \alpha^{2} \tau_{p-1}}$. If we redefine the gauge field as $a_{\alpha} \rightarrow a_{\alpha}+b_{\alpha} \phi$ and make nonabelianization, we obtain from (6.6)

$$
\frac{1}{g_{Y M}^{2}} \int d^{p} x \sqrt{g} \operatorname{tr}\left(\frac{1}{4}\left(F_{\alpha \beta}-b_{\alpha \beta} \phi\right)^{2}+\frac{1}{2}\left(D_{\alpha} \phi\right)^{2}\right),
$$

which indeed agrees with (3.20) with $G=U(1)$.

\section{Acknowledgements}

We would like to thank T. Higashi for discussions. The work of G.I. is supported in part by the JSPS Research Fellowship for Young Scientists. The work of A.T. is supported in part by Grant-in-Aid for Scientific Research (No. 19540294) from the Ministry of Education, Culture, Sports, Science and Technology.

\section{A Spherical harmonics}

In this appendix, we review the spherical harmonics on $S^{3}$, the monopole harmonics on $S^{2}$ [42] and the fuzzy spherical harmonics [15, 24,49]. For more details, see [29, 48] and references therein.

\section{A.1 Spherical harmonics on $S^{3}$}

We regard $S^{3}$ as the $S U(2)$ group manifold. We parameterize an element of $S U(2)$ in terms of the Euler angles as

$$
g=e^{-i \varphi J_{3}} e^{-i \theta J_{2}} e^{-i \psi J_{3}},
$$


where $J_{A}$ satisfy $\left[J_{A}, J_{B}\right]=i \epsilon_{A B C} J_{C}$ and $0 \leq \theta \leq \pi, 0 \leq \varphi<2 \pi, 0 \leq \psi<4 \pi$. The isometry of $S^{3}$ is $S O(4)=S U(2) \times S U(2)$, and these two $S U(2)$ 's act on $g$ from left and right, respectively. We construct the right invariant 1-forms,

$$
d g g^{-1}=-i \mu E^{A} J_{A}
$$

where the radius of $S^{3}$ is $2 / \mu$. They are explicitly given by

$$
\begin{aligned}
E^{1} & =\frac{1}{\mu}(-\sin \varphi d \theta+\sin \theta \cos \varphi d \psi), \\
E^{2} & =\frac{1}{\mu}(\cos \varphi d \theta+\sin \theta \sin \varphi d \psi), \\
E^{3} & =\frac{1}{\mu}(d \varphi+\cos \theta d \psi),
\end{aligned}
$$

and satisfy the Maure-Cartan equation

$$
d E^{A}-\frac{\mu}{2} \epsilon_{A B C} E^{B} \wedge E^{C}=0 .
$$

The metric is constructed from $E^{A}$ as

$$
d s^{2}=E^{A} E^{A}=\frac{1}{\mu^{2}}\left(d \theta^{2}+\sin ^{2} \theta d \varphi^{2}+(d \psi+\cos d \varphi)^{2}\right) .
$$

The Killing vectors dual to $E^{A}$ are given by

$$
\mathcal{L}_{A}=-\frac{i}{\mu} E_{A}^{M} \partial_{M}
$$

where $E_{A}^{M}$ are inverse of $E_{M}^{A}$. The explicit form of the Killing vectors are

$$
\begin{aligned}
& \mathcal{L}_{1}=-i\left(-\sin \varphi \partial_{\theta}-\cot \theta \cos \varphi \partial_{\varphi}+\frac{\cos \varphi}{\sin \theta} \partial_{\psi}\right), \\
& \mathcal{L}_{2}=-i\left(\cos \varphi \partial_{\theta}-\cot \theta \sin \varphi \partial_{\varphi}+\frac{\sin \varphi}{\sin \theta} \partial_{\psi}\right), \\
& \mathcal{L}_{3}=-i \partial_{\varphi} .
\end{aligned}
$$

Because of the Maure-Cartan equation (A.4), the Killing vectors satisfy the $\mathrm{SU}(2)$ algebra, $\left[\mathcal{L}_{A}, \mathcal{L}_{B}\right]=i \epsilon_{A B C} \mathcal{L}_{C}$

The scalar spherical harmonics on $S^{3}$ are given by

$$
Y_{J m \tilde{m}}\left(\Omega_{3}\right)=(-1)^{J-\tilde{m}} \sqrt{2 J+1}\left\langle J-\tilde{m}\left|g^{-1}\right| J m\right\rangle .
$$

These spherical harmonics form the basis of $\mathrm{SU}(2)$ algebra generated by $\mathcal{L}_{A}$ 's.

$$
\mathcal{L}^{2} Y_{J m \tilde{m}}=J(J+1) Y_{J m \tilde{m}},
$$




$$
\begin{aligned}
\mathcal{L}_{ \pm} Y_{J m \tilde{m}} & =\sqrt{(J \mp m)(J \pm m+1)} Y_{J m \pm 1 \tilde{m}} \\
\mathcal{L}_{3} Y_{J m \tilde{m}} & =m Y_{J m \tilde{m}} .
\end{aligned}
$$

The complex conjugates of the spherical harmonics are evaluated as

$$
\left(Y_{J m \tilde{m}}\right)^{*}=(-1)^{m-\tilde{m}} Y_{J-m-\tilde{m}}
$$

The spherical harmonics also satisfy the orthonormality condition

$$
\int \frac{d \Omega_{3}}{2 \pi^{2}}\left(Y_{J m \tilde{m}}\right)^{*} Y_{J^{\prime} m^{\prime} \tilde{m}^{\prime}}=\delta_{J J^{\prime}} \delta_{m m^{\prime}} \delta_{\tilde{m} \tilde{m}^{\prime}}
$$

The integral of the product of three spherical harmonics is given as follows:

$$
\begin{aligned}
\mathcal{C}_{J_{2} m_{2} \tilde{m}_{2} J_{3} m_{3} \tilde{m}_{3}}^{J_{1} m_{1} \tilde{m}_{1}} & \equiv \int \frac{d \Omega_{3}}{2 \pi^{2}}\left(Y_{J_{1} m_{1} \tilde{m}_{1}}\right)^{*} Y_{J_{2} m_{2} \tilde{m}_{2}} Y_{J_{3} m_{3} \tilde{m}_{3}} \\
& =\sqrt{\frac{\left(2 J_{2}+1\right)\left(2 J_{3}+1\right)}{2 J_{1}+1}} C_{J_{2} m_{2} J_{3} m_{3}}^{J_{1} m_{1}} C_{J_{2} \tilde{m}_{2} J_{3} \tilde{m}_{3}}^{J_{1} \tilde{m}_{1}},
\end{aligned}
$$

where $C_{J_{2} m_{2} J_{3} m_{3}}^{J_{1} m_{1}}$ is the Clebsch-Gordan coefficient of $S U(2)$. Finally, the spherical harmonics satisfy the completeness condition,

$$
\sum_{J m \tilde{m}}\left(Y_{J m \tilde{m}}\right)^{*}\left(\Omega_{3}\right) Y_{J m \tilde{m}}\left(\Omega_{3}^{\prime}\right)=2 \pi^{2} \delta\left(\Omega_{3}-\Omega_{3}^{\prime}\right)
$$

where

$$
\delta\left(\Omega_{3}\right)=\frac{8}{\sin \theta} \delta(\theta) \delta(\varphi) \delta(\psi)
$$

\section{A.2 Monopole spherical harmonics on $S^{2}$}

We adopt the following metric for $S^{2}$ :

$$
d s^{2}=\frac{1}{\mu^{2}}\left(d \theta^{2}+\sin ^{2} \theta d \varphi^{2}\right) .
$$

We define two local patches on $S^{2}$ to describe nontrivial $U(1)$ bundles over $S^{2}$ : the patch I is specified by $0 \leq \theta<\pi$ and the patch II is specified by $0<\theta \leq \pi$. In the following expressions, the upper sign is taken in the patch I and the lower sign in the patch II.

The angular momentum operator in the presence of a monopole with magnetic charge $q$ at the origin takes the form

$$
L_{1}^{(q)}=i\left(\sin \varphi \partial_{\theta}+\cot \theta \cos \varphi \partial_{\varphi}\right)-q \frac{1 \mp \cos \theta}{\sin \theta} \cos \varphi
$$




$$
\begin{aligned}
& L_{2}^{(q)}=i\left(-\cos \varphi \partial_{\theta}+\cot \theta \sin \varphi \partial_{\varphi}\right)-q \frac{1 \mp \cos \theta}{\sin \theta} \sin \varphi, \\
& L_{3}^{(q)}=-i \partial_{\varphi} \mp q,
\end{aligned}
$$

where $q$ is quantized as $q=0, \pm \frac{1}{2}, \pm 1, \pm \frac{3}{2}, \cdots$. These operators act on the local sections on $S^{2}$ and satisfy the $S U(2)$ algebra $\left[L_{A}^{(q)}, L_{B}^{(q)}\right]=i \epsilon_{A B C} L_{C}^{(q)}$. Note that when $q=0$, these operators are reduced to the ordinary angular momentum operators on $S^{2}$ (or $R^{3}$ ). if we regard $S^{3}$ as a $U(1)$ bundle over $S^{2}$, and parameterize the fiber direction by $y=\psi \pm \varphi$, the above expression (A.16) can be obtained by making a replacement in (A.7): $\partial_{y} \rightarrow-i q$.

The monopole spherical harmonics are the basis of local sections on $S^{2}$ and also form the basis of the $S U(2)$ algebra generated by $L_{A}^{(q)}$. The monopole scalar spherical harmonics are given by

$$
\tilde{Y}_{J m q}\left(\Omega_{2}\right)=(-1)^{J-q} \sqrt{2 J+1}\left\langle J-q\left|e^{i \theta J_{2}}\right| J m\right\rangle e^{i( \pm q+m) \varphi} .
$$

Here $J=|q|,|q|+1,|q+2|, \cdots, m=-J,-J+1, \cdots, J-1, J$. The existence of the lower bound of the angular momentum $J$ is due to the fact that the magnetic field produced by the monopole also has nonzero angular momentum. Note that the monopole harmonics with $q=0$ do not transform on the overlap of two patches. They correspond to global sections (functions) on $S^{2}$ which are expressed by the ordinary spherical harmonics on $S^{2}$. The action of $L_{A}^{(q)}$ on the monopole spherical harmonics is given by

$$
\begin{aligned}
L^{(q) 2} \tilde{Y}_{J m q} & =J(J+1) \tilde{Y}_{J m q}, \\
L_{ \pm}^{(q)} \tilde{Y}_{J m q} & =\sqrt{(J \mp m)(J \pm m+1)} \tilde{Y}_{J m \pm 1 q} \\
L_{3}^{(q)} \tilde{Y}_{J m q} & =m \tilde{Y}_{J m q} .
\end{aligned}
$$

The complex conjugates of the monopole spherical harmonics are evaluated as

$$
\left(\tilde{Y}_{J m q}\right)^{*}=(-1)^{m-q} \tilde{Y}_{J-m-q}
$$

The monopole spherical harmonics are orthonormal to each other,

$$
\int \frac{d \Omega_{2}}{4 \pi}\left(\tilde{Y}_{J m q}\right)^{*} \tilde{Y}_{J^{\prime} m^{\prime} q}=\delta_{J J^{\prime}} \delta_{m m^{\prime}}
$$

The integral of three monopole spherical harmonics is equal to the corresponding integral (A.12) on $S^{3}$ with the identification $\tilde{m}=q$,

$$
\begin{aligned}
\int \frac{d \Omega_{2}}{4 \pi}\left(\tilde{Y}_{J_{1} m_{1} q_{1}}\right)^{*} \tilde{Y}_{J_{2} m_{2} q_{2}} \tilde{Y}_{J_{3} m_{3} q_{3}} & =\sqrt{\frac{\left(2 J_{2}+1\right)\left(2 J_{3}+1\right)}{2 J_{1}+1}} C_{J_{2} m_{2} J_{3} m_{3}}^{J_{1} m_{1}} C_{J_{2} q_{2} J_{3} q_{3}}^{J_{1} q_{1}} \\
& =\mathcal{C}_{J_{2} m_{2} q_{2} J_{3} m_{3} q_{3}}^{J_{1} m_{1} q_{1}}
\end{aligned}
$$


where the monopole charges must be conserved in the lefthand side of the above equation as $q_{1}+q_{2}+q_{3}=0$. Note that the monopole spherical harmonics are expressed in terms of the spherical harmonics on $S^{3}$ :

$$
\begin{aligned}
\tilde{Y}_{J m q}\left(\Omega_{2}\right) & =e^{i q(\psi \pm \varphi)} Y_{J m q}\left(\Omega_{3}\right), \\
L_{A}^{(q)} \tilde{Y}_{J m q}\left(\Omega_{2}\right) & =e^{i q(\psi \pm \varphi)} \mathcal{L}_{A} Y_{J m q}\left(\Omega_{3}\right) .
\end{aligned}
$$

(A.21) and (A.22) represent a map between the local sections on $S^{2}$ and the Kaluza-Klein modes on $S^{3}$.

\section{A.3 Fuzzy spherical harmonics}

Let us consider $(2 j+1) \times\left(2 j^{\prime}+1\right)$ rectangular complex matrices. Such matrices are generally expressed as

$$
M=\sum_{r, r^{\prime}} M_{r r^{\prime}}|j r\rangle\left\langle j^{\prime} r^{\prime}\right|
$$

We can define linear maps $\hat{L}_{A^{\circ}}$, which map the set of $(2 j+1) \times\left(2 j^{\prime}+1\right)$ rectangular complex matrices to itself, by their operation on the basis:

$$
\hat{L}_{A} \circ|j r\rangle\left\langle j^{\prime} r^{\prime}\left|\equiv \hat{L}_{A}^{[j]}\right| j r\right\rangle\left\langle j^{\prime} r^{\prime}|-| j r\right\rangle\left\langle j^{\prime} r^{\prime}\right| \hat{L}_{A}^{\left[j^{\prime}\right]}
$$

where $\hat{L}_{A}^{[j]}$ are the spin $j$ representation matrices of the $S U(2)$ generators. $\hat{L}_{A}$ o satisfy the $S U(2)$ algebra $\left[\hat{L}_{A^{\circ}}, \hat{L}_{B} \circ\right]=i \epsilon_{A B C} \hat{L}_{C}$.

We make a change of a basis of the rectangular matrices from the above basis $\left\{|j r\rangle\left\langle j^{\prime} r^{\prime}\right|\right\}$ to the new basis which is called the fuzzy spherical harmonics:

$$
\hat{Y}_{J m\left(j j^{\prime}\right)}=\sqrt{N_{0}} \sum_{r, r^{\prime}}(-1)^{-j+r^{\prime}} C_{j r j^{\prime}-r^{\prime}}^{J m}|j r\rangle\left\langle j^{\prime} r^{\prime}\right|
$$

where $N_{0}$ is a positive integer which will be specified below. For a fixed $J$ the fuzzy spherical harmonics also form a basis of the spin $J$ irreducible representation of $S U(2)$ which is generated by $\hat{L}_{A}$,

$$
\begin{aligned}
\left(\hat{L}_{A} \circ\right)^{2} \hat{Y}_{J m\left(j j^{\prime}\right)} & =J(J+1) \hat{Y}_{J m\left(j j^{\prime}\right)}, \\
\hat{L}_{ \pm} \circ \hat{Y}_{J m\left(j j^{\prime}\right)} & =\sqrt{(J \mp m)(J \pm m+1)} \hat{Y}_{J m \pm 1\left(j j^{\prime}\right)}, \\
\hat{L}_{3} \circ \hat{Y}_{J m\left(j j^{\prime}\right)} & =m \hat{Y}_{J m\left(j j^{\prime}\right)} .
\end{aligned}
$$

The hermitian conjugates of the fuzzy spherical harmonics are evaluated as

$$
\left(\hat{Y}_{J m\left(j j^{\prime}\right)}\right)^{\dagger}=(-1)^{m-\left(j-j^{\prime}\right)} \hat{Y}_{J-m\left(j^{\prime} j\right)}
$$


The fuzzy spherical harmonics satisfy the orthonormality condition under the following normalized trace:

$$
\frac{1}{N_{0}} \operatorname{tr}\left\{\left(\hat{Y}_{J m\left(j j^{\prime}\right)}\right)^{\dagger} \hat{Y}_{J^{\prime} m^{\prime}\left(j j^{\prime}\right)}\right\}=\delta_{J J^{\prime}} \delta_{m m^{\prime}}
$$

where tr stands for the trace over $\left(2 j^{\prime}+1\right) \times\left(2 j^{\prime}+1\right)$ matrices. The trace of three fuzzy spherical harmonics is given by

$$
\begin{aligned}
\hat{C}_{J_{2} m_{2}\left(j j^{\prime}\right) J_{3} m_{3}\left(j^{\prime} j^{\prime \prime}\right)}^{J_{1} m_{1}\left(j j^{\prime \prime}\right.} & \equiv \frac{1}{N_{0}} \operatorname{tr}\left\{\left(\hat{Y}_{J_{1} m_{1}\left(j j^{\prime \prime}\right)}\right)^{\dagger} \hat{Y}_{J_{2} m_{2}\left(j j^{\prime}\right)} \hat{Y}_{J_{3} m_{3}\left(j^{\prime} j^{\prime \prime}\right)}\right\} \\
& =(-1)^{J_{1}+j+j^{\prime \prime}} \sqrt{N_{0}\left(2 J_{2}+1\right)\left(2 J_{3}+1\right)} C_{J_{2} m_{2} J_{3} m_{3}}^{J_{1} m_{1}}\left\{\begin{array}{ccc}
J_{1} & J_{2} & J_{3} \\
j^{\prime} & j^{\prime \prime} & j
\end{array}\right\},
\end{aligned}
$$

where the last factor of the above equation is the $6-j$ symbol.

In order to reveal relationships among the fuzzy spherical harmonics, the monopole harmonics on $S^{2}$ and the spherical harmonics on $S^{3}$, we introduce the following parameterization for $j, j^{\prime}$ and $j^{\prime \prime}$,

$$
2 j+1=N_{0}+\zeta, 2 j^{\prime}+1=N_{0}+\zeta^{\prime}, 2 j^{\prime \prime}+1=N_{0}+\zeta^{\prime \prime} .
$$

$\zeta, \zeta^{\prime}$ and $\zeta^{\prime \prime}$ are integers which are grater than $-N_{0}$. Then, in the limit $N_{0} \rightarrow \infty$, one can show that

$$
\hat{C}_{J_{2} m_{2}\left(j j^{\prime}\right) J_{3} m_{3}\left(j^{\prime} j^{\prime \prime}\right)}^{J_{1} m_{1}\left(j j^{\prime \prime}\right)} \rightarrow \mathcal{C}_{J_{2} m_{2} q_{2} J_{3} m_{3} q_{3}}^{J_{1} m_{1} q_{1}}
$$

with the identification $j-j^{\prime \prime}=q_{1}, j-j^{\prime}=q_{2}$ and $j^{\prime}-j^{\prime \prime}=q_{3}$. This relation can be proved by using the following asymptotic form of the $6-j$ symbols. If $R \gg 1$, one obtains [50]

$$
\left\{\begin{array}{ccc}
a & b & c \\
d+R & e+R & f+R
\end{array}\right\} \approx \frac{(-1)^{a+b+c+2(d+e+f+R)}}{\sqrt{2 R}}\left(\begin{array}{ccc}
a & b & c \\
e-f & f-d & d-e
\end{array}\right),
$$

where the $3-j$ symbol is related to the Clebsch-Gordan coefficient as

$$
\left(\begin{array}{ccc}
J_{1} & J_{2} & J_{3} \\
m_{1} & m_{2} & m_{3}
\end{array}\right)=(-1)^{J_{3}+m_{3}+2 J_{1}} \frac{1}{\sqrt{2 J_{3}+1}} C_{J_{1}-m_{1} J_{2}-m_{2}}^{J_{3} m_{3}}
$$

The relation (A.31) implies that the fuzzy spherical harmonics $\hat{Y}_{J m\left(j j^{\prime}\right)}$ give a matrix regularization of the monopole harmonics $\tilde{Y}_{J m q}$ through the following correspondence:

$$
\begin{aligned}
j-j^{\prime} & \leftrightarrow q, \\
\hat{L}_{A} \circ & \leftrightarrow L_{A}^{(q)} \\
\frac{1}{N_{0}} \operatorname{tr} & \leftrightarrow \int \frac{d \Omega_{2}}{4 \pi} .
\end{aligned}
$$

Furthermore, combining the above correspondence and the relations (A.21) and (A.22), we can also map the fuzzy spherical harmonics to the spherical harmonics on $S^{3}$. 


\section{A.4 Vector spherical harmonics}

We introduce vector spherical harmonics for three different types of the spherical harmonics that we have defined above. The vector spherical harmonics are given by

$$
\begin{aligned}
Y_{J m \tilde{m} A}^{\rho}\left(\Omega_{3}\right) & =i^{\rho} \sum_{n, p} U_{A n} C_{\tilde{Q} p 1 n}^{Q m} Y_{\tilde{Q} p \tilde{m}}\left(\Omega_{3}\right), \\
\tilde{Y}_{J m q A}^{\rho}\left(\Omega_{2}\right) & =i^{\rho} \sum_{n, p} U_{A n} C_{\tilde{Q} p 1 n}^{Q m} \tilde{Y}_{\tilde{Q} p q}\left(\Omega_{2}\right), \\
\hat{Y}_{J m\left(j j^{\prime}\right) A}^{\rho} & =i^{\rho} \sum_{n, p} U_{A n} C_{\tilde{Q} p 1 n}^{Q m} \hat{Y}_{\tilde{Q} p\left(j j^{\prime}\right)},
\end{aligned}
$$

where $\rho=-1,0,1$ and $Q=J+\delta_{\rho 1}, \tilde{Q}=J+\delta_{\rho-1}$. These spherical harmonics transform as the vector representations under $S U(2)$ rotation. The unitary matrix $U$ is given by

$$
U=\left(\begin{array}{ccc}
-1 & 0 & 1 \\
-i & 0 & -i \\
0 & \sqrt{2} & 0
\end{array}\right)
$$

The vector spherical harmonics satisfy

$$
\begin{aligned}
& \frac{1}{\mu} \epsilon_{A B C} \nabla_{B} Y_{J m \tilde{m} C}^{\rho}=i \epsilon_{A B C} \mathcal{L}_{B} Y_{J m \tilde{m} C}^{\rho}+Y_{J m \tilde{m} A}^{\rho}=\rho(J+1) Y_{J m \tilde{m} A}^{\rho}, \\
& i \epsilon_{A B C} L_{B}^{(q)} \tilde{Y}_{J m q C}^{\rho}+\tilde{Y}_{J m q A}^{\rho}=\rho(J+1) \tilde{Y}_{J m q A}^{\rho}, \\
& i \epsilon_{A B C} \hat{L}_{B} \circ \hat{Y}_{J m\left(j j^{\prime}\right) C}^{\rho}+\hat{Y}_{J m\left(j j^{\prime}\right) A}^{\rho}=\rho(J+1) \hat{Y}_{J m\left(j j^{\prime}\right) A}^{\rho} .
\end{aligned}
$$

The complex (hermitian) conjugates of these vector harmonics are evaluated as

$$
\begin{aligned}
\left(Y_{J m \tilde{m} A}^{\rho}\right)^{*} & =(-1)^{m-\tilde{m}+1} Y_{J-m-\tilde{m} A}^{\rho}, \\
\left(\tilde{Y}_{J m q A}^{\rho}\right)^{*} & =(-1)^{m-q+1} \tilde{Y}_{J-m-q A}^{\rho}, \\
\left(\hat{Y}_{J m\left(j j^{\prime}\right) A}^{\rho}\right)^{\dagger} & =(-1)^{m-\left(j-j^{\prime}\right)+1} \hat{Y}_{J-m\left(j^{\prime} j\right) A}^{\rho} .
\end{aligned}
$$

The orthonormal relations are

$$
\begin{aligned}
\int \frac{d \Omega_{3}}{2 \pi^{2}}\left(Y_{J m \tilde{m} A}^{\rho}\right)^{*} Y_{J^{\prime} m^{\prime} \tilde{m}^{\prime} A}^{\rho^{\prime}} & =\delta_{J J^{\prime}} \delta_{m m^{\prime}} \delta_{\tilde{m} \tilde{m}^{\prime}} \delta_{\rho \rho^{\prime}}, \\
\int \frac{d \Omega_{2}}{4 \pi}\left(\tilde{Y}_{J m q A}^{\rho}\right)^{*} \tilde{Y}_{J^{\prime} m^{\prime} q A}^{\rho^{\prime}} & =\delta_{J J^{\prime}} \delta_{m m^{\prime}} \delta_{\rho \rho^{\prime}}, \\
\frac{1}{N_{0}} \operatorname{tr}\left(\left(\hat{Y}_{J m\left(j j^{\prime}\right) A}^{\rho}\right)^{\dagger} \hat{Y}_{J^{\prime} m^{\prime}\left(j^{\prime} j\right) A}^{\rho^{\prime}}\right) & =\delta_{J J^{\prime}} \delta_{m m^{\prime}} \delta_{\rho \rho^{\prime}} .
\end{aligned}
$$

Finally, the integrals (or trace) of three vector harmonics are given by

$$
\int \frac{d \Omega_{3}}{2 \pi^{2}} \epsilon_{A B C} Y_{J_{1} m_{1} \tilde{m}_{1} A}^{\rho_{1}} Y_{J_{2} m_{2} \tilde{m}_{2} B}^{\rho_{2}} Y_{J_{3} m_{3} \tilde{m}_{3} C}^{\rho_{3}}=\mathcal{E}_{J_{1} m_{1} \tilde{m}_{1} \rho_{1} J_{2} m_{2} \tilde{m}_{2} \rho_{2} J_{3} m_{3} \tilde{m}_{3} \rho_{3}},
$$




$$
\begin{aligned}
\int \frac{d \Omega_{2}}{4 \pi} \epsilon_{A B C} \tilde{Y}_{J_{1} m_{1} q_{1} A}^{\rho_{1}} \tilde{Y}_{J_{2} m_{2} q_{2} B}^{\rho_{2}} \tilde{Y}_{J_{3} m_{3} q_{3} C}^{\rho_{3}} & =\mathcal{E}_{J_{1} m_{1} q_{1} \rho_{1} J_{2} m_{2} q_{2} \rho_{2} J_{3} m_{3} q_{3} \rho_{3}}, \\
\epsilon_{A B C} \frac{1}{N_{0}} \operatorname{tr}\left(\hat{Y}_{J_{1} m_{1}\left(j j^{\prime}\right) A}^{\rho_{1}} \hat{Y}_{J_{2} m_{2}\left(j^{\prime} j^{\prime \prime}\right) B}^{\rho_{2}} \hat{Y}_{J_{3} m_{3}\left(j^{\prime \prime} j\right) C}^{\rho_{3}}\right) & =\hat{\mathcal{E}}_{J_{1} m_{1}\left(j j^{\prime}\right) \rho_{1} J_{2} m_{2}\left(j^{\prime} j^{\prime \prime}\right) \rho_{2} J_{3} m_{3}\left(j^{\prime \prime} j\right) \rho_{3}},
\end{aligned}
$$

where the monopole charges must be conserved in the lefthand side of the second equality as $q_{1}+q_{2}+q_{3}=0$ and $\mathcal{E}, \hat{\mathcal{E}}$ are given by

$$
\begin{aligned}
& \mathcal{E}_{J_{1} m_{1} \tilde{m}_{1} \rho_{1} J_{2} m_{2} \tilde{m}_{2} \rho_{2} J_{3} m_{3} \tilde{m}_{3} \rho_{3}} \\
& =\sqrt{6\left(2 J_{1}+1\right)\left(2 J_{1}+2 \rho_{1}^{2}+1\right)\left(2 J_{2}+1\right)\left(2 J_{2}+2 \rho_{2}^{2}+1\right)\left(2 J_{3}+1\right)\left(2 J_{3}+2 \rho_{3}^{2}+1\right)} \\
& \quad \times(-1)^{-\frac{\rho_{1}+\rho_{2}+\rho_{3}+1}{2}}\left\{\begin{array}{lll}
Q_{1} & \tilde{Q}_{1} & 1 \\
Q_{2} & \tilde{Q}_{2} & 1 \\
Q_{3} & \tilde{Q}_{3} & 1
\end{array}\right\}\left(\begin{array}{ccc}
Q_{1} & Q_{2} & Q_{3} \\
m_{1} & m_{2} & m_{3}
\end{array}\right)\left(\begin{array}{ccc}
\tilde{Q}_{1} & \tilde{Q}_{2} & \tilde{Q}_{3} \\
\tilde{m}_{1} & \tilde{m}_{2} & \tilde{m}_{3}
\end{array}\right), \\
& \hat{\mathcal{E}}_{J_{1} m_{1}\left(j j^{\prime}\right) \rho_{1} J_{2} m_{2}\left(j^{\prime} j^{\prime \prime}\right) \rho_{2} J_{3} m_{3}\left(j^{\prime \prime} j\right) \rho_{3}}(\mathrm{~A} .41) \\
& =\sqrt{6 N_{0}\left(2 J_{1}+1\right)\left(2 J_{1}+2 \rho_{1}^{2}+1\right)\left(2 J_{2}+1\right)\left(2 J_{2}+2 \rho_{2}^{2}+1\right)\left(2 J_{3}+1\right)\left(2 J_{3}+2 \rho_{3}^{2}+1\right)} \\
& \quad \times(-1)^{-\frac{\rho_{1}+\rho_{2}+\rho_{3}+1}{2}-\tilde{Q}_{1}-\tilde{Q}_{2}-\tilde{Q}_{3}+2 j+2 j^{\prime}+2 j^{\prime \prime}}\left\{\begin{array}{lll}
Q_{1} & \tilde{Q}_{1} & 1 \\
Q_{2} & \tilde{Q}_{2} & 1 \\
Q_{3} & \tilde{Q}_{3} & 1
\end{array}\right\}\left(\begin{array}{ccc}
Q_{1} & Q_{2} & Q_{3} \\
m_{1} & m_{2} & m_{3}
\end{array}\right)\left\{\begin{array}{ccc}
\tilde{Q}_{1} & \tilde{Q}_{2} & \tilde{Q}_{3} \\
j^{\prime \prime} & j & j^{\prime}
\end{array}\right\} .
\end{aligned}
$$

As in (A.31), we can show

$$
\hat{\mathcal{E}}_{J_{1} m_{1}\left(j j^{\prime}\right) \rho_{1} J_{2} m_{2}\left(j^{\prime} j^{\prime \prime}\right) \rho_{2} J_{3} m_{3}\left(j^{\prime \prime} j\right) \rho_{3}} \rightarrow \mathcal{E}_{J_{1} m_{1} \tilde{m}_{1} \rho_{1} J_{2} m_{2} \tilde{m}_{2} \rho_{2} J_{3} m_{3} \tilde{m}_{3} \rho_{3}}
$$

in the limit $N_{0} \rightarrow \infty$ with $j-j^{\prime}=q_{1}, j^{\prime}-j^{\prime \prime}=q_{2}$ and $j^{\prime \prime}-j=q_{3}$ fixed.

\section{B Derivation of (4.22)}

In this appendix, we give the derivation of (4.22) in some detail.

$$
\begin{aligned}
{\left[L_{a}, a_{\alpha}(x)\right]^{(s, t)} } & =a_{\alpha, J m}^{\left(q_{s t}\right)}(x) \otimes L_{a} \circ \hat{Y}_{J m\left(j_{s} j_{t}\right)} \\
& =\int \frac{d \Omega_{3}}{2 \pi^{2}} A_{\alpha}(z) Y_{J m q_{s t}}^{\dagger}(y) \otimes L_{a} \circ \hat{Y}_{J m\left(j_{s} j_{t}\right)} \\
& =\int \frac{d \Omega_{3}}{2 \pi^{2}} A_{\alpha}(z) \mathcal{L}_{a} Y_{J m q_{s t}}^{\dagger} \otimes \hat{Y}_{J m\left(j_{s} j_{t}\right)} \\
& =\int \frac{d \Omega_{3}}{2 \pi^{2}}\left(\mathcal{L}_{a} A_{\alpha}(z)\right) Y_{J m q_{s t}}^{\dagger} \otimes \hat{Y}_{J m\left(j_{s} j_{t}\right)},
\end{aligned}
$$


where we have used (A.9) and (A.26).

$$
\begin{aligned}
& {\left[\phi_{a}, \phi_{b}\right]^{(s, t)}=\sum_{u}\left(\phi_{a, J m}^{\left(q_{s u}\right)} \phi_{b, J^{\prime} m^{\prime}}^{\left(q_{u u t}\right)}-\phi_{b, J m}^{\left(q_{s u}\right)} \phi_{a, J^{\prime} m^{\prime}}^{\left(q_{u t}\right)}\right) \otimes \hat{Y}_{J m\left(j_{s} j_{u}\right)} \hat{Y}_{J^{\prime} m^{\prime}\left(j_{u} j_{t}\right)}} \\
& =\sum_{u}\left(\phi_{a, J m}^{\left(q_{s u}\right)} \phi_{b, J^{\prime} m^{\prime}}^{\left(q_{u t}\right)}-\phi_{b, J m}^{\left(q_{s u}\right)} \phi_{a, J^{\prime} m^{\prime}}^{\left(q_{u t}\right)}\right) \otimes \hat{\mathcal{C}}_{J m\left(j_{s} j_{u}\right) J^{\prime \prime} m^{\prime}\left(j_{u} j_{t}\right)}^{J^{\prime \prime}\left(j_{s} j_{t}\right)} \hat{Y}_{J^{\prime \prime} m^{\prime \prime}\left(j_{s} j_{t}\right)} \\
& =\sum_{u, v} \int \frac{d \Omega_{3}}{2 \pi^{2}} \frac{d \Omega_{3}^{\prime}}{2 \pi^{2}}\left\{A_{a}(z) A_{b}\left(z^{\prime}\right)-A_{b}(z) A_{a}\left(z^{\prime}\right)\right\} Y_{J m q_{s u}}^{\dagger}(y) Y_{J^{\prime} m^{\prime} q_{v t}}^{\dagger}\left(y^{\prime}\right) \\
& \times \int \frac{d \Omega_{3}^{\prime \prime}}{2 \pi^{2}} Y_{J^{\prime \prime} m^{\prime \prime} q_{s t}}^{\dagger}\left(y^{\prime \prime}\right) Y_{J m q_{s u}}\left(y^{\prime \prime}\right) Y_{J^{\prime} m^{\prime} q_{v t}}\left(y^{\prime \prime}\right) \otimes \hat{Y}_{J^{\prime \prime} m^{\prime \prime}\left(j_{s} j_{t}\right)} \\
& =\int \frac{d \Omega_{3}}{2 \pi^{2}}\left[A_{a}(z), A_{b}(z)\right] Y_{J m q_{s t}}^{\dagger}(y) \otimes \hat{Y}_{J m\left(j_{s} j_{t}\right)} .
\end{aligned}
$$

In the third and fourth lines of the righthand side, we have used (A.31), the charge conservation $\tilde{m}^{\prime \prime}=\tilde{m}+\tilde{m}^{\prime}$ of $\mathcal{C}_{J m \tilde{m}}^{J^{\prime \prime} m_{J^{\prime} m^{\prime}}^{\prime \prime} \tilde{m}^{\prime \prime}}$ and $(\underline{\mathrm{A} .12})$, so that we have added the new summation

over $v$ additionally and replaced $q_{u t}$ by $q_{v t}$. Then, we can regard the summation $\sum_{u, v}$ as $\sum_{q_{s u}, q_{v t}}$, and the last equality holds due to (A.13).

\section{Group manifold and coset space}

In this appendix, we describe some conventions on the group manifold $\tilde{G}$ and the coset space $\tilde{G} / H$ which we follow in this paper.

We parameterize an element of $\tilde{G}$ as

$$
g(z)=L(x) h(y)
$$

where $L(x) \in \tilde{G} / H, h(y) \in H$, the coordinates $z^{M}, x^{\mu}$ and $y^{m}$ parameterize $\tilde{G}, \tilde{G} / H$ and $H$ respectively and $z^{M}$ are decomposed into $\left(x^{\mu}, y^{i}\right)$. We can construct the right and left invariant 1 -forms on $\tilde{G}$ as

$$
d g g^{-1}=-i E_{R}^{A} T^{A}, \quad g^{-1} d g=i E_{L}^{A} T^{A}
$$

where $A=1, \cdots, \operatorname{dim} \tilde{G}$ and $T^{A}$ represent the generators of $\tilde{G}$ which satisfy the Lie algebra of $\tilde{G},\left[T^{A}, T^{B}\right]=i f_{A B C} T^{C}$. We decompose $T^{A}$ into $\left(T^{\alpha}, T^{a}\right)$ where $\alpha=1, \cdots, \operatorname{dim} \tilde{G} / H, a=$ $\operatorname{dim} \tilde{G} / H+1, \cdots, \operatorname{dim} \tilde{G}$, and we assume that $T^{a}$ satisfy the Lie algebra of $H$ which is a subalgebra of $\tilde{G},\left[T^{a}, T^{b}\right]=i f_{a b c} T^{c}$. The both of $E_{R}^{A}$ and $E_{L}^{A}$ satisfy the Maurer-Cartan equation,

$$
d E_{R}^{A}-\frac{1}{2} f_{A B C} E_{R}^{B} \wedge E_{R}^{C}=0, \quad d E_{L}^{A}-\frac{1}{2} f_{A B C} E_{L}^{B} \wedge E_{L}^{C}=0
$$


We also introduce the right and left invariant 1-form for $L(x) \in \tilde{G} / H$ and $h(y) \in H$ as follows:

$$
\begin{aligned}
d L L^{-1} & =-i\left(e_{R}\right)_{\mu}^{A}(x) T^{A} d x^{\mu}, \quad L^{-1} d L=i\left(e_{L}\right)_{\mu}^{A}(x) T^{A} d x^{\mu} \\
d h h^{-1} & =-i\left(\tilde{e}_{R}\right)_{m}^{a}(y) T^{a} d y^{m}, \quad h^{-1} d h=i\left(\tilde{e}_{L}\right)_{m}^{a}(y) T^{a} d y^{m} .
\end{aligned}
$$

Then, we can write down the components of $E_{R}^{A}$ and $E_{L}^{A}$ explicitly:

$$
\left(E_{R}\right)^{A}{ }_{M}=\left(\begin{array}{cc}
\left(e_{R}\right)_{\mu}^{\alpha} & \operatorname{Ad}(L)^{\alpha}{ }_{b}\left(\tilde{e}_{R}\right)_{m}^{b} \\
\left(e_{R}\right)_{\mu}^{a} & \operatorname{Ad}(L)^{a}{ }_{b}\left(\tilde{e}_{R}\right)_{m}^{b}
\end{array}\right), \quad\left(E_{L}\right)^{A}{ }_{M}=\left(\begin{array}{cc}
\left(e_{L}\right)_{\mu}^{\beta} \operatorname{Ad}(h)_{\beta \alpha} & 0 \\
\left(e_{L}\right)_{\mu}^{\beta} \operatorname{Ad}(h)_{\beta a} & \left(\tilde{e}_{L}\right)_{m}^{a}
\end{array}\right),
$$

where Ad is defined as the adjoint action $g T^{A} g^{-1}=T^{B} \operatorname{Ad}(g)_{B A}$. The Cartan-Killing metric on $\tilde{G}$ is defined as

$$
d s^{2}=G_{M N} d z^{M} d z^{N}=-2 \operatorname{Tr}\left(d g g^{-1} d g g^{-1}\right) .
$$

In terms of the components (C.5), the above metric is written as

$$
d s^{2}=\left(e_{L}\right)_{\mu}^{\alpha}\left(e_{L}\right)_{\nu}^{\alpha} d x^{\mu} d x^{\nu}+\left\{\left(\tilde{e}_{R}\right)_{m}^{a} d y^{m}-\left(e_{L}\right)_{\mu}^{a} d x^{\mu}\right\}^{2} .
$$

We can regard the group manifold $\tilde{G}$ as the principal $H$ bundle on $\tilde{G} / H$. By comparing (C.7) and (3.5), therefore, we can make the following identifications:

$$
\left(e_{L}\right)_{\mu}^{\alpha}\left(e_{L}\right)_{\nu}^{\alpha}=g_{\mu \nu},\left(e_{L}\right)_{\mu}^{a}=b_{\mu}^{a},\left(\tilde{e}_{R}\right)_{m}^{a}\left(\tilde{e}_{R}\right)_{n}^{a}=h_{m n},
$$

where $g_{\mu \nu}$ and $h_{m n}$ are the metrics on $\tilde{G} / H$ and $H$, respectively, and $b_{\mu}^{a}$ are the local connection 1-forms of the principal $H$ bundle. Namely, we can regard $\left(e_{L}\right)_{\mu}^{\alpha}$ and $\left(\tilde{e}_{R}\right)_{m}^{a}$ as the vielbein on $\tilde{G} / H$ and $H$, respectively. The metric (C.6) is invariant under the right and left actions of $\tilde{G}$. The corresponding right and left invariant Killing vectors on $\tilde{G}$ are defined in terms of the inverse of $E^{A}$ as

$$
\mathcal{L}_{A}^{R}=-i\left(E_{R}\right)_{A}^{M} \partial_{M}, \quad \mathcal{L}_{A}^{L}=-i\left(E_{L}\right)_{A}^{M} \partial_{M}
$$

By using (C.3), we can show that $\mathcal{L}_{A}^{R}$ and $\mathcal{L}_{A}^{L}$ satisfy the Lie algebra of $\tilde{G} \times \tilde{G}$,

$$
\left[\mathcal{L}_{A}^{R}, \mathcal{L}_{B}^{R}\right]=i f_{A B C} \mathcal{L}_{C}^{R}, \quad\left[\mathcal{L}_{A}^{L}, \mathcal{L}_{B}^{L}\right]=i f_{A B C} \mathcal{L}_{C}^{L}, \quad\left[\mathcal{L}_{A}^{R}, \mathcal{L}_{B}^{L}\right]=0
$$

and they also satisfy the Killing vector equations,

$$
\nabla_{M} \mathcal{L}_{A N}+\nabla_{N} \mathcal{L}_{A M}=0
$$

where $\nabla_{M}$ are the covariant derivative on $\tilde{G}$ and $\mathcal{L}_{A M}=G_{M N} \mathcal{L}_{A}^{N}$. We also define the following operators:

$$
L_{A}=-i\left(E_{R}\right)_{A}^{\mu} \partial_{\mu}
$$

One can show that $L_{A}$ do not depend on $y^{m}$ and they satisfy $\left[L_{A}, L_{B}\right]=i f_{A B C} L_{C}$ by using (C.10). Furthermore, we can show that

$$
\nabla_{\mu}^{(\tilde{G} / H)} L_{A \nu}+\nabla_{\nu}^{(\tilde{G} / H)} L_{A \mu}=0
$$

where $L_{A \mu}=g_{\mu \nu} L_{A}^{\nu}$. Namely, $L_{A}$ are the Killing vectors on the coset space $\tilde{G} / H$. 


\section{Metrics of $S U(3), S^{5}$ and $C P^{2}$}

In this appendix, for concreteness, we give an explicit form of the metrics of $S U(3), S U(3) / S U(2) \simeq$ $S^{5}$ and $S U(3) /(S U(2) \times U(1)) \simeq C P^{2}$ [51]. We parameterize an element $g$ of $S U(3)$ as

$$
g=L(\chi, \theta, \varphi, \psi) Z(\tau) V(a, b, c)
$$

where

$$
\begin{aligned}
& L(\chi, \theta, \varphi, \psi)=e^{i \varphi \lambda_{3}} e^{i \theta \lambda_{2}} e^{i \psi \lambda_{3}} e^{2 i \chi \lambda_{5}}, \\
& Z(\tau)=e^{-i \sqrt{3}(\tau-2 \pi) \lambda_{8}} \\
& V(a, b, c)=e^{-i a \lambda_{3}} e^{-i b \lambda_{2}} e^{-i c \lambda_{3}}
\end{aligned}
$$

and $0 \leq \chi \leq \frac{\pi}{2}, 0 \leq \theta \leq \pi, 0 \leq \varphi<2 \pi, 0 \leq \psi<4 \pi, 0 \leq \tau<2 \pi, 0 \leq a<2 \pi, 0 \leq b \leq \pi$ and $0 \leq c<4 \pi . \lambda_{1}, \cdots, \lambda_{8}$ are the Gell-Mann matrices and satisfy $\operatorname{Tr}\left(\lambda_{a} \lambda_{b}\right)=\frac{1}{2} \delta_{a b}$. The metric of $S U(3)$ is given by

$$
\begin{aligned}
d S_{S U(3)}^{2}= & -\frac{1}{2} \operatorname{Tr}\left(d g g^{-1} d g g^{-1}\right) \\
= & d \chi^{2}+\frac{1}{4} \sin ^{2} \chi\left\{d \theta^{2}+\sin ^{2} \theta d \varphi^{2}+\cos ^{2} \chi(d \psi+\cos \theta d \varphi)^{2}\right\} \\
& +\frac{3}{4}\left\{d \tau+\frac{1}{2} \sin ^{2} \chi(d \psi+\cos \theta d \varphi)\right\}^{2} \\
& +\frac{1}{4}\left\{e^{1}+\cos \chi(\sin \psi d \theta-\sin \theta \cos \psi d \varphi)\right\}^{2} \\
& +\frac{1}{4}\left\{e^{2}-\cos \chi(\cos \psi d \theta+\sin \theta \sin \psi d \varphi)\right\}^{2} \\
& +\frac{1}{4}\left\{e^{3}-\frac{1}{2}\left(1+\cos ^{2} \chi\right)(d \psi+\cos \theta d \varphi)\right\}^{2},
\end{aligned}
$$

where

$$
\begin{aligned}
& e^{1}=-\sin a d b+\cos a \sin b d c, \\
& e^{2}=\cos a d b+\sin a \sin b d c, \\
& e^{3}=d a+\cos b d c
\end{aligned}
$$

which are the right invariant 1-form of $S U(2)$. $S U(3)$ is an $S U(2) \times U(1)$ bundle over $C P^{2}$. The second line in the righthand side of (D.3) is the Fubini-Study metric of $C P^{2}$. The third line represents the $U(1)$ fiber structure while the fourth, fifth and sixth lines represent the $S U(2)$ fiber structure. $S U(3)$ is also viewed as an $S U(2)$ bundle over $S^{5} \simeq S U(3) / S U(2)$. The second and third lines together correspond to the metric of $S^{5} \simeq S U(3) / S U(2) . S^{5} \simeq$ 
$S U(3) / S U(2)$ is viewed as a $U(1)$ bundle over $C P^{2}$. The metric of the ordinary unit $S^{5}$ is given by the sum of the second and third lines with the factor $3 / 4$ in the third line replaced by $1 / 4$.

\section{E Fuzzy $C P^{n}$}

In this appendix, we give a brief review of a construction of fuzzy $C P^{n}[28,31-35]$.

\section{E.1 Functions on fuzzy $C P^{n}$}

Fuzzy $C P^{n}$ is a well-known example of noncommutative space which is given by the quantization of coadjoint orbit of $S U(n+1)$ in terms of a certain matrix algebra acting on an appropriate representation space $V$. We can determine this matrix algebra and the representation space $V$ by matching the spectrum of functions on $C P^{n}$ and that on fuzzy $C P^{n}$.

In order to consider the spectrum of functions on $C P^{n}$, We regard $C P^{n}$ as a coadjoint orbit in the Lie algebra of $S U(n+1)$.

$$
C P^{n}=\left\{g t g^{-1} \mid g \in S U(n+1)\right\} \simeq S U(n+1) /(S U(n) \times U(1)),
$$

where $t$ is an element of the $S U(n+1)$ Lie algebra such that the stabilizer of $t$ is given by $S U(n) \times U(1)$. For example, for the case of $C P^{2}$, we can take $t$ to be $\lambda_{8}$ which is invariant under $S U(2) \times U(1)$ adjoint action generated by $\lambda_{1}, \lambda_{2}, \lambda_{3}$ and $\lambda_{8}$. Functions on $C P^{n}$ should be invariant under the action of $S U(n) \times U(1)$. Then, the space of functions on $C P^{n}$ is given by a direct sum of the representation spaces of $S U(n+1)$ which contain $S U(n) \times U(1)$ invariant states:

$$
C^{\infty}\left(C P^{n}\right)=\bigoplus_{J=0}^{\infty} V_{[J, 0, \cdots, 0, J]}
$$

where we denote $[J, 0, \cdots, 0, J]$ as the Dynkin index of $S U(n+1)$, and $V_{[J, 0, \cdots, 0, J]}$ represents the corresponding irreducible representation space of the $S U(n+1)$ Lie algebra. One can show that $V_{[J, 0, \cdots, 0, J]}$ are the only spaces which contain the $S U(n) \times U(1)$ singlets.

The space of functions on fuzzy $C P^{n}$ is obtained by introducing a cutoff $\Lambda$ in (E.2) as

$$
\bigoplus_{J=0}^{\Lambda} V_{[J, 0, \cdots, 0, J]}=V_{[\Lambda, 0, \cdots, 0]} \otimes V_{[\Lambda, 0, \cdots, 0]}^{*} \cdot
$$

By definition, it is obvious that the above spectrum on fuzzy $C P^{n}$ tends to the spectrum (E.2) on $C P^{n}$ in the commutative limit $\Lambda \rightarrow \infty$. Note that the righthand side of the above equation can be viewed as a space of matrices. From this viewpoint, we make an 
identification $V=V_{[\Lambda, 0, \cdots, 0]}$ and regard functions on fuzzy $C P^{n}$ as matrices acting on the vector space $V$. In particular, the coordinates on fuzzy $C P^{n}$ are identified with

$$
\hat{\xi}_{A}=\hat{L}_{A}^{[\Lambda, 0, \cdots, 0]},
$$

which are the generators of $S U(n+1)$ in the irreducible representation specified by the Dynkin index $[\Lambda, 0, \cdots, 0]$. These coordinates on fuzzy $C P^{n}$ are actually reduced to the coordinates on $C P^{n}$ in the commutative limit through a map which will be defined in the last part of this section.

\section{E.2 Derivatives on fuzzy $C P^{n}$}

In order to construct differential operators on fuzzy $C P^{n}$, let us recall the simplest case of fuzzy $C P^{1} \simeq S^{2}$. In this case, we established the differential operators on fuzzy $S^{2}$ in appendix A. As shown in (A.34), the adjoint action of the $S U(2)$ generators is reduced to the action of the Killing vectors on $S^{2}$ in the commutative limit. We can generalize this fact into the case of fuzzy $C P^{n}$ with $n \geq 2$. The adjoint action of the $S U(n+1)$ generators on the space of square matrices $(\mathrm{E} .3),\left[\hat{L}_{A}^{[\Lambda, 0, \cdots, 0]}, \cdot\right]$, is mapped into the action of the Killing vectors on the space of functions on $C P^{n}$ in the commutative limit.

\section{E.3 $U(1)$ monopoles on fuzzy $C P^{n}$}

Topologically nontrivial field configurations including $U(1)$ monopoles can be realized on fuzzy $C P^{n}$. If we consider rectangular matrices in addition to the square matrices (E.3), the concept of fiber bundles naturally arises. Let us again consider the case of fuzzy $C P^{1}$. We have shown in appendix A that the basis of $(2 j+1) \times\left(2 j^{\prime}+1\right)$ rectangular matrices, $\hat{Y}_{J m\left(j j^{\prime}\right)}$, are mapped into local sections of the $U(1)$ fiber bundle on $S^{2}$. In this correspondence, The difference $j-j^{\prime}$ is identified with the monopole charge $q$ of the $U(1)$ bundle. This fact is also generalized into the case of $C P^{n}$ with $n \geq 2$. For the case of $C P^{n}$, we consider a space of rectangular matrices,

$$
V_{[\Lambda+q, 0, \cdots, 0,]} \otimes V_{[\Lambda-q, 0, \cdots, 0,]}^{*} .
$$

Here, the charge $q$ is a half integer and we take $\Lambda \pm q$ to be integers. When $q=0, \Lambda$ is an integer and this is the case of square matrices (E.3). We can show that elements of (E.5) are mapped into local sections of $U(1)$ fiber bundle on $C P^{n}$ with the monopole charge $q$. Furthermore, we can extend the action of the differential operators $\left[\hat{L}_{A}^{[\Lambda, 0, \cdots, 0]}, \cdot\right]$ discussed above to the action on rectangular matrices as follows.

$$
\hat{L}_{A} \circ \hat{M}_{q}=\hat{L}_{A}^{[\Lambda+q, 0, \cdots, 0]} \hat{M}_{q}-\hat{M}_{q} \hat{L}_{A}^{[\Lambda-q, 0, \cdots, 0]},
$$


where $\hat{M}_{q}$ is an element of (E.5). When $q=0, \hat{M}_{0}$ is just a square matrix and $\hat{L}_{A}$ ○ are nothing but the commutators $\left[\hat{L}_{A}^{[\Lambda, 0, \cdots, 0]}, \cdot\right]$. The operators $\hat{L}_{A} \circ$ map the space (E.5) to itself and they are reduced to the angular momentum operators in the presence of a $U(1)$ monopole with the magnetic charge $q$ in the commutative limit. We will show these facts in the following subsections.

\section{E.4 Fock space representation}

In order to construct a map between matrices and functions on $C P^{n}$, we introduce the Fock space representation developed in [35]. Let $a_{\alpha}^{\dagger}, \alpha=1,2, \cdots, n+1$ be a set of creation operators and $a^{\alpha}$ be a set of annihilation operators which annihilate the Fock vacuum $|0\rangle$. They satisfy the Heisenberg commutation relations.

$$
\left[a^{\alpha}, a^{\beta}\right]=\left[a_{\alpha}^{\dagger}, a_{\beta}^{\dagger}\right]=0, \quad\left[a^{\alpha}, a_{\beta}^{\dagger}\right]=\delta_{\beta}^{\alpha} .
$$

By acting the creation operators on the vacuum state $|0\rangle$, we can construct the entire Fock space $\mathcal{F}$ which is spanned by

$$
\left|p_{1}, p_{2}, \cdots, p_{n+1}\right\rangle=\frac{1}{\sqrt{p_{1} ! p_{2} ! \cdots p_{n+1} !}}\left(a_{1}^{\dagger}\right)^{p_{1}}\left(a_{2}^{\dagger}\right)^{p_{2}} \cdots\left(a_{n+1}^{\dagger}\right)^{p_{n+1}}|0\rangle .
$$

In terms of the operators (E.7), we can construct elements of the Lie algebra of $S U(n+1)$,

$$
\hat{L}_{A}=a_{\alpha}^{\dagger}\left(T_{A}\right)^{\alpha}{ }_{\beta} a^{\beta}
$$

where $T_{A}=\hat{L}_{A}^{[1,0, \cdots, 0]}$ represent the generators of $S U(n+1)$ in the fundamental representation. We also define the number operator which commutes with all the operators in (E.9).

$$
\hat{N}=a_{\alpha}^{\dagger} a^{\alpha}
$$

The operators (E.9) and (E.10) act on the Fock space $\mathcal{F}$, and satisfy

$$
\left[\hat{L}_{A}, \hat{L}_{B}\right]=i f_{A B C} \hat{L}_{C}, \quad\left[\hat{L}_{A}, \hat{N}\right]=0
$$

We can decompose the Fock space $\mathcal{F}$ into the eigenspaces of $\hat{N}$ as

$$
\mathcal{F}=\bigoplus_{p=0}^{\infty} V_{[p, 0, \cdots, 0]}
$$

where $p$ represent an eigenvalue of $\hat{N}$. The basis of each eigenspace $V_{[p, 0, \cdots, 0]}$ is formed by

$$
\left|\boldsymbol{\alpha}_{p}\right\rangle=\left|\alpha_{1}, \alpha_{2}, \cdots, \alpha_{p}\right\rangle=\frac{1}{\sqrt{p !}} a_{\alpha_{1}}^{\dagger} a_{\alpha_{2}}^{\dagger} \cdots a_{\alpha_{p}}^{\dagger}|0\rangle,
$$


where $\boldsymbol{\alpha}_{p}$ is an abbreviation of a set of $p$ indices, $\left(\alpha_{1}, \alpha_{2}, \cdots, \alpha_{p}\right)$.

Let us consider square matrices which are elements of (E.3). These matrices are generally written as

$$
\hat{M}=\hat{M}_{\boldsymbol{\beta}_{\Lambda}}^{\boldsymbol{\alpha}_{\Lambda}}\left|\boldsymbol{\alpha}_{\Lambda}\right\rangle\left\langle\boldsymbol{\beta}_{\Lambda}\right|
$$

We define a new basis of these matrices to see the correspondence with the spectrum of functions on $C P^{n},(\underline{\mathrm{E} .2})$ :

$$
\hat{Y}_{\boldsymbol{\beta}_{J}} \boldsymbol{\alpha}_{J}=N_{\Lambda J}^{n} \mathcal{P}_{\boldsymbol{\beta}_{J}, \boldsymbol{\tau}_{J}} \boldsymbol{\alpha}_{J}, \boldsymbol{\sigma}_{J}\left|\boldsymbol{\sigma}_{J}, \boldsymbol{\gamma}_{\Lambda-J}\right\rangle\left\langle\boldsymbol{\tau}_{J}, \gamma_{\Lambda-J}\right|
$$

where $\Lambda-J$ indices $\gamma_{\Lambda-J}$ are contracted and $\mathcal{P}_{\boldsymbol{\beta}_{J}, \boldsymbol{\tau}_{J}} \boldsymbol{\alpha}_{J}, \boldsymbol{\sigma}_{J}$ is the projection operator onto the representation space $V_{[J, 0, \cdots, 0, J]}$ which appeared in the decomposition (E.3), that is, it removes all traces between $\boldsymbol{\alpha}_{J}$ and $\boldsymbol{\beta}_{J}$. For example,

$$
\hat{Y}=N_{\Lambda 0}^{n} \mathbf{1}, \quad \hat{Y}_{\beta}^{\alpha}=N_{\Lambda 1}^{n}\left(\left|\beta, \gamma_{\Lambda-1}\right\rangle\left\langle\alpha, \gamma_{\Lambda-1}\right|-\frac{1}{2} \delta_{\beta}^{\alpha} \mathbf{1}\right) .
$$

Hence, $\hat{Y}_{\boldsymbol{\beta}_{J}} \boldsymbol{\alpha}_{J}$ belong to the representation $V_{[J, 0, \cdots, 0, J]}$ and they are mapped to the corresponding spherical harmonics on $C P^{n}$ in the commutative limit which are elements of $(\overline{\mathrm{E} .2}) \cdot N_{\Lambda J}^{n}$ is an appropriate normalization constant which is determined by the following orthonormality of the basis,

$$
\operatorname{tr}\left(\left(\hat{Y}_{\boldsymbol{\beta}_{J}}^{\boldsymbol{\alpha}_{J}}\right)^{\dagger} \hat{Y}_{\boldsymbol{\tau}_{J^{\prime}}}^{\boldsymbol{\sigma}_{J^{\prime}}}\right)=\delta_{J J^{\prime}} \mathcal{P}_{\boldsymbol{\alpha}_{J}, \boldsymbol{\tau}_{J}} \boldsymbol{\beta}_{J}, \boldsymbol{\sigma}_{J}
$$

In the case of $n=1, \hat{Y}_{\boldsymbol{\beta}_{J}} \boldsymbol{\alpha}_{J}$ are essentially the same as the fuzzy spherical harmonics which are defined in (A.25). The action of differential operators on fuzzy $C P^{n}$ is given by the adjoint action of operators in (E.9). Then, one can evaluate the eigenvalues of the Laplacian as follows:

$$
\left[\hat{L}_{A},\left[\hat{L}_{A}, \hat{Y}_{\boldsymbol{\beta}_{J}}^{\boldsymbol{\alpha}_{J}}\right]\right]=J(J+n) \hat{Y}_{\boldsymbol{\beta}_{J}}^{\boldsymbol{\alpha}_{J}} .
$$

The above spectrum completely matches the spectrum of functions on $C P^{n}$ up to the cutoff $\Lambda$.

In terms of the Fock space representation, we can also express rectangular matrices which are elements of (E.5) . Those rectangular matrices are generally expressed as

$$
\hat{M}_{q}=\left(\hat{M}_{q}\right)^{\boldsymbol{\alpha}_{\Lambda+q}} \boldsymbol{\beta}_{\Lambda-q}\left|\boldsymbol{\alpha}_{\Lambda+q}\right\rangle\left\langle\boldsymbol{\beta}_{\Lambda-q}\right|
$$

These matrices are expanded by a similar basis to (E.15). Note that the direct product representation (E.5) is decomposed as

$$
\bigoplus_{J=|q|}^{\Lambda} V_{[J+q, 0, \cdots, 0, J-q]}
$$


For each representation space in $(\underline{E .20})$ with fixed $J$, we can use the following basis:

$$
\hat{Y}_{\boldsymbol{\beta}_{J+q}}^{(q)} \boldsymbol{\alpha}_{J-q}=N_{\Lambda J q}^{n} \mathcal{P}_{\boldsymbol{\beta}_{J+q}, \boldsymbol{\tau}_{J-q}} \boldsymbol{\alpha}_{J-q}, \boldsymbol{\sigma}_{J+q}\left|\boldsymbol{\sigma}_{J+q}, \boldsymbol{\gamma}_{\Lambda-J}\right\rangle\left\langle\boldsymbol{\tau}_{J-q}, \boldsymbol{\gamma}_{\Lambda-J}\right|
$$

As in the case of square matrices, $\mathcal{P}_{\boldsymbol{\beta}_{J+q}, \boldsymbol{\tau}_{J-q}} \boldsymbol{\alpha}_{J-q}, \boldsymbol{\sigma}_{J+q}$ is a projection operator onto the space (E.20) with fixed $J$ and $N_{\Lambda J q}^{n}$ is a normalization constant which is determined by

$$
\operatorname{tr}\left(\left(\hat{Y}^{(q)} \boldsymbol{\beta}_{J+q} \boldsymbol{\alpha}_{J-q}\right)^{\dagger} \hat{Y}^{(q)} \underset{\boldsymbol{\tau}_{J^{\prime}+q}}{\boldsymbol{\sigma}_{J^{\prime}-q}}\right)=\delta_{J J^{\prime}} \mathcal{P}_{\boldsymbol{\tau}_{J+q}, \boldsymbol{\alpha}_{J-q}} \boldsymbol{\sigma}_{J-q}, \boldsymbol{\beta}_{J+q}
$$

When $q=0, \hat{Y}^{(0)}{ }_{\boldsymbol{\beta}_{J}}{ }^{\boldsymbol{\alpha}_{J}}$ are identical with the square matrices (E.15). The action of differential operators on $\hat{Y}^{(q)} \boldsymbol{\beta}_{J+q}{ }^{\boldsymbol{\alpha}_{J-q}}$ is given by (E.6) . We can evaluate the eigenvalues of the Laplacian as follows:

$$
\left(\hat{L}_{A^{\circ}}\right)^{2} \hat{Y}^{(q)}{ }_{\boldsymbol{\beta}_{J+q}}{ }^{\boldsymbol{\alpha}_{J-q}}=\left(J(J+1)+\frac{n-1}{n+1} q^{2}\right) \hat{Y}_{\boldsymbol{\beta}_{J+q}^{(q)}}^{\boldsymbol{\alpha}_{J-q} .}
$$

The above spectrum is the same as the spectrum of local sections of $U(1)$ bundle on $C P^{n}$ up to the cutoff. We show in the following that the rectangular matrices $\hat{Y}^{(q)} \boldsymbol{\beta}_{J+q} \boldsymbol{\alpha}_{J-q}$ are indeed mapped to the local sections on $C P^{n}$.

\section{E.5 Relation between matrices and sections}

Let us recall the spherical harmonics on $C P^{n}$. In a spinorial basis, they are given by

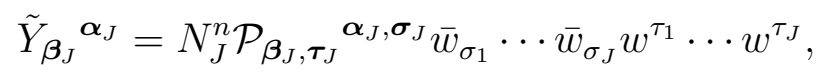

In the above expression, $w^{\alpha}$ are the coordinates of $S^{2 n+1} \simeq S U(n+1) / S U(n)$ which satisfy $\sum_{\alpha}\left|w^{\alpha}\right|^{2}=1$ and the normalization constant $N_{J}^{n}$ is determined by

$$
\int_{C P^{n}} \omega^{n}\left(\tilde{Y}_{\boldsymbol{\beta}_{J}}^{\boldsymbol{\alpha}_{J}}\right)^{*} \tilde{Y}_{\boldsymbol{\tau}_{J^{\prime}}}^{\boldsymbol{\sigma}_{J^{\prime}}}=\delta_{J J^{\prime}} \mathcal{P}_{\boldsymbol{\alpha}_{J}, \boldsymbol{\tau}_{J}}^{\boldsymbol{\beta}_{J}, \boldsymbol{\sigma}_{J}}
$$

where $\omega^{n}$ is the volume form on $C P^{n}$. The functions (E.24) are invariant under the $U(1)$ phase rotation so that they can be regarded as global sections (functions) on $C P^{n}$. We can generalize (E.24) to a basis of local sections of the $U(1)$ monopole bundle on $C P^{n}$. The local sections of the monopole bundle with the magnetic charge $q$ can be expanded by

$$
\tilde{Y}_{\boldsymbol{\beta}_{J+q}}^{(q)}{ }^{\boldsymbol{\alpha}_{J-q}}=N_{J q}^{n} \mathcal{P}_{\boldsymbol{\beta}_{J+q}, \boldsymbol{\tau}_{J-q}} \boldsymbol{\alpha}_{J-q}, \boldsymbol{\sigma}_{J+q} \bar{w}_{\sigma_{1}} \cdots \bar{w}_{\sigma_{J+q}} w^{\tau_{1}} \cdots w^{\tau_{J-q}}
$$

which are normalized as

$$
\int_{C P^{n}} \omega^{n}\left(\tilde{Y}^{(q)} \boldsymbol{\beta}_{J+q}{ }^{\boldsymbol{\alpha}_{J-q}}\right)^{*} \tilde{Y}^{(q)}{\boldsymbol{\boldsymbol { \tau } _ { J ^ { \prime } + q }}}^{\boldsymbol{\sigma}_{J^{\prime}-q}}=\delta_{J J^{\prime}} \mathcal{P}_{\boldsymbol{\alpha}_{J-q}, \boldsymbol{\tau}_{J+q}}^{\boldsymbol{\beta}_{J+q}, \boldsymbol{\sigma}_{J-q}}
$$


$\tilde{Y}^{(q)} \boldsymbol{\beta}_{J+q} \boldsymbol{\alpha}_{J-q}$ are not invariant under the $U(1)$ phase rotation, so that they transform as the local sections of the monopole bundle on $C P^{n}$ with the magnetic charge $q$. Note that $\tilde{Y}^{(0)} \boldsymbol{\beta}_{J} \boldsymbol{\alpha}_{J}$ are nothing but the global sections, $\tilde{Y}_{\boldsymbol{\beta}_{J}} \boldsymbol{\alpha}_{J}$.

The relation between matrices and sections on $C P^{n}$ is given by the diagonal coherent state map [35]. Let us consider a matrix $\hat{M}_{q}$ which is an element of (E.5) and expanded as in (E.19). $\hat{M}_{q}$ corresponds to a section of the monopole bundle on $C P^{n}$ through the map. In particular, when $q=0, \hat{M}_{0}$ is just a square matrix and corresponds to a global section on $C P^{n}$. The map to the sections is given by

$$
\tilde{M}_{q}(w, \bar{w})=\left\langle w, \Lambda+q\left|\hat{M}_{q}\right| w, \Lambda-q\right\rangle,
$$

where

$$
|w, p\rangle=\frac{1}{\sqrt{p !}}\left(w^{\alpha} a_{\alpha}^{\dagger}\right)^{p}|0\rangle .
$$

The map (E.28) is equivalent to the following replacement up to an over all constant factor,

$$
\begin{aligned}
& \left(a_{\alpha}^{\dagger}\right)^{L} \rightarrow \bar{w}_{\alpha}, \quad\left(a^{\alpha}\right)^{L} \rightarrow \frac{\partial}{\partial \bar{w}_{\alpha}}, \\
& \left(a^{\alpha}\right)^{R} \rightarrow w^{\alpha}, \quad\left(a_{\alpha}^{\dagger}\right)^{R} \rightarrow \frac{\partial}{\partial w^{\alpha}},
\end{aligned}
$$

where the superscripts $L$ and $R$ express that the operators act on matrices from the left and right, respectively. Through this correspondence, (E.15) and (E.21) are mapped to (E.24) and (E.26) respectively. Furthermore, the differential operators $\hat{L}_{A^{\circ}}$ on fuzzy $C P^{n}$ are mapped to

$$
\hat{L}_{A^{\circ}} \rightarrow L_{A}^{(q)}=\bar{w}_{\alpha}\left(T_{A}\right)^{\alpha}{ }_{\beta} \frac{\partial}{\partial \bar{w}_{\beta}}-w^{\alpha}\left(T_{A}^{*}\right)_{\alpha}{ }^{\beta} \frac{\partial}{\partial w^{\beta}} .
$$

When $q=0$, these operators act on the functions (E.24) and they can be identified with the Killing vectors on $C P^{n}$. In the case $q \neq 0$, however, they act on the local sections (E.26) so that the derivative along the $U(1)$ fiber direction does not vanish and yields additional terms which are proportional to the charge $q$. In this case, the operators (E.31) can be interpreted as the angular momentum operators on $C P^{n}$ in the presence of a monopole with the charge $q$.

\section{References}

[1] T. Banks, W. Fischler, S. H. Shenker and L. Susskind, Phys. Rev. D 55 (1997) 5112 arXiv:hep-th/9610043].

[2] N. Ishibashi, H. Kawai, Y. Kitazawa and A. Tsuchiya, Nucl. Phys. B 498 (1997) 467 arXiv:hep-th/9612115. 
[3] R. Dijkgraaf, E. P. Verlinde and H. L. Verlinde, Nucl. Phys. B 500 (1997) 43 arXiv:hep-th/9703030.

[4] T. Eguchi and H. Kawai, Phys. Rev. Lett. 48 (1982) 1063.

[5] G. Bhanot, U. M. Heller and H. Neuberger, Phys. Lett. B 113, 47 (1982).

[6] G. Parisi, Phys. Lett. B 112, 463 (1982).

[7] D. J. Gross and Y. Kitazawa, Nucl. Phys. B 206, 440 (1982).

[8] S. R. Das and S. R. Wadia, Phys. Lett. B 117 (1982) 228 [Erratum-ibid. B 121 (1983) 456].

[9] A. Gonzalez-Arroyo and M. Okawa, Phys. Rev. D 27 (1983) 2397.

[10] H. Aoki, N. Ishibashi, S. Iso, H. Kawai, Y. Kitazawa and T. Tada, Nucl. Phys. B 565 (2000) 176 arXiv:hep-th/9908141.

[11] W. I. Taylor, Phys. Lett. B 394 (1997) 283 arXiv:hep-th/9611042].

[12] N. Arkani-Hamed, A. G. Cohen and H. Georgi, Phys. Lett. B 513, 232 (2001) arXiv:hep-ph/0105239.

[13] D. B. Kaplan, E. Katz and M. Unsal, JHEP 0305, 037 (2003) arXiv:hep-lat/0206019.

[14] M. Hanada, H. Kawai and Y. Kimura, Prog. Theor. Phys. 114 (2006) 1295 arXiv:hep-th/0508211.

[15] G. Ishiki, S. Shimasaki, Y. Takayama and A. Tsuchiya, JHEP 0611 (2006) 089 arXiv:hep-th/0610038.

[16] J. Maldacena, M. M. Sheikh-Jabbari and M. Van Raamsdonk, JHEP 0301 (2003) 038 arXiv:hep-th/0211139.

[17] D. Berenstein, J. M. Maldacena and H. Nastase, JHEP 0204 (2002) 013 arXiv:hep-th/0202021.

[18] H. Lin and J. M. Maldacena, Phys. Rev. D 74, 084014 (2006) arXiv:hep-th/0509235.

[19] J. Madore, Class. Quant. Grav. 9 (1992) 69.

[20] H. Grosse and J. Madore, Phys. Lett. B 283 (1992) 218. 
[21] H. Grosse, C. Klimcik and P. Presnajder, Int. J. Theor. Phys. 35 (1996) 231 arXiv:hep-th/9505175.

[22] U. Carow-Watamura and S. Watamura, Commun. Math. Phys. 212 (2000) 395 arXiv:hep-th/9801195.

[23] S. Iso, Y. Kimura, K. Tanaka and K. Wakatsuki, Nucl. Phys. B 604 (2001) 121 arXiv:hep-th/0101102.

[24] H. Grosse, C. Klimcik and P. Presnajder, Commun. Math. Phys. 178 (1996) 507 arXiv:hep-th/9510083.

[25] S. Baez, A. P. Balachandran, B. Ydri and S. Vaidya, Commun. Math. Phys. 208 (2000) 787 arXiv:hep-th/9811169].

[26] G. Landi, J.Geom.Phys. 37 (2001) 47.

[27] H. Aoki, S. Iso and K. Nagao, Nucl. Phys. B 684 (2004) 162 arXiv:hep-th/0312199.

[28] U. Carow-Watamura, H. Steinacker and S. Watamura, J. Geom. Phys. 54 (2005) 373 arXiv:hep-th/0404130.

[29] T. Ishii, G. Ishiki, S. Shimasaki and A. Tsuchiya, JHEP 0705 (2007) 014 arXiv:hep-th/0703021.

[30] T. Ishii, G. Ishiki, K. Ohta, S. Shimasaki and A. Tsuchiya, arXiv:0711.4235 [hep-th].

[31] G. Alexanian, A. P. Balachandran, G. Immirzi and B. Ydri, J. Geom. Phys. 42, 28 (2002) arXiv:hep-th/0103023.

[32] A. P. Balachandran, B. P. Dolan, J. H. Lee, X. Martin and D. O'Connor, J. Geom. Phys. 43, 184 (2002) arXiv:hep-th/0107099.

[33] Y. Kitazawa, Nucl. Phys. B 642, 210 (2002) arXiv:hep-th/0207115.

[34] H. Grosse and H. Steinacker, Nucl. Phys. B 707, 145 (2005) arXiv:hep-th/0407089.

[35] B. P. Dolan, I. Huet, S. Murray and D. O'Connor, JHEP 0707, 007 (2007) arXiv:hep-th/0611209.

[36] T. H. Buscher, Phys. Lett. B 194, 59 (1987).

[37] X. C. de la Ossa and F. Quevedo, Nucl. Phys. B 403 (1993) 377 arXiv:hep-th/9210021. 
[38] T. Azuma, S. Bal, K. Nagao and J. Nishimura, JHEP 0605 (2006) 061 arXiv:hep-th/0405277.

[39] T. Ishii, G. Ishiki, S. Shimasaki and A. Tsuchiya, to appear.

[40] R. C. Myers, JHEP 9912 (1999) 022 [arXiv:hep-th/9910053].

[41] N. w. Kim, T. Klose and J. Plefka, Nucl. Phys. B $671 \quad$ (2003) 359 arXiv:hep-th/0306054.

[42] T. T. Wu and C. N. Yang, Nucl. Phys. B 107 (1976) 365.

[43] Y. Choquet-Bruhat, Analysis, Manifolds and Physics, Part II (North Holland, 2000).

[44] M. Nakahara, "Geometry, topology and physics," Boca Raton, USA: Taylor \& Francis (2003) $573 \mathrm{p}$.

[45] G. L. Naber, "Topology, geometry, and gauge fields: Foundations," New York, USA: Springer (1997) 396 p. (Texts in applied mathematics. 25).

[46] G. L. Naber, "Topology, geometry, and gauge fields: Interactions," New York, USA: Springer (2000) $443 \mathrm{p}$.

[47] C. N. Yang, J. Math. Phys. 19, 320 (1978).

[48] G. Ishiki, Y. Takayama and A. Tsuchiya, JHEP $\mathbf{0 6 1 0}$ (2006) 007 arXiv:hep-th/0605163].

[49] K. Dasgupta, M. M. Sheikh-Jabbari and M. Van Raamsdonk, "Matrix perturbation theory for M-theory on a PP-wave," JHEP 0205 (2002) 056 [arXiv:hep-th/0205185].

[50] D. Varshalovich, A. Moskalev and V. Khersonskii, Quantum Theory of Angular Momentum (World Scientific, Singapore, 1988).

[51] V. Gerdt, R. Horan, A. Khvedelidze, M. Lavelle, D. McMullan and Yu. Palii, J. Math. Phys. 47, 112902 (2006) arXiv:hep-th/0511245. 